LBL-34459

UC-402

\title{
ROLE OF SURFACE CHARACTERISTICS IN URBAN METEOROLOGY AND AIR QUALITY
}

\author{
David Jean Sailor \\ Ph.D. Thesis \\ Mechanical Engineering Department \\ University of California \\ and \\ Energy and Environment Division \\ Lawrence Berkeley Laboratory \\ University of California \\ Berkeley, CA 94720
}

August, 1993

This work was supported by the California Institute for Energy Efficiency (CIEE) through the U.S. Department of Energy, under contract No. DE-ACOB-765F00098. 


\section{Table of Contents}

Chapter 1: Introduction to the Urban Climate ............................................ 1

Historical Background .............................................................................. 1

The Urban Fabric ........................................................................... 3

Characterization ................................................................................. 4

Urban Heat Islands ........................................................................... 5

Emissions .............................................................................................. 6

Photochemical Smog ....................................................................... 7

Mitigation ............................................................................................. 9

Chapter 2: Review of Urban Meteorological Modeling …............................ 14

Urban Energy Balance ........................................................................... 14

Current State of Urban Climate Models ................................................. $\quad 25$

Validation and Comparison of Models .................................................. $\quad 36$

Climate Models and Builciing Energy Use .............................................. 38

Summary ............................................................................................... 42

Chapter 3: Surface Characteristic Sensitivity Experiments ........................... 50

Introduction ....................................................................................... 50

Methodology ..................................................................................... 51

Base Case ............................................................................................ 54

Parametric Analysis ........................................................................ 55

Results ........................................................................................... 58

Summary of Sensitivity Results .... ..................................................... $\quad 72$

Chapter 4: Accurate Representation of the Urban Surface ............................ 83

Data Requirements ............................................................................. 83

Land Use Databases ............................................................................... 84

Remoiely-Sensed Radiometry Data ...................................................... $\quad 88$

Surface Characteristic Parameterization ................................................. 91

Antluropogenic Heating ....................................................................... 110

Chapter 5: Meteorological Modeling of the Los Angeles Basin .................... 117

Introduction ................................................................................... 117

Homogeneous Surface Case .............................................................. 123

UTZ Heterogeneous Case ................................................................ 127

Anthropogenic Heating Case ............................................................... 130

Mitigation Scenario Development ....................................................... 131 
Moderate Level Modification Results ...................................................... 136

Extreme Level Modification Results ....................................................... 140

Combined Modification Results .............................................................. 141

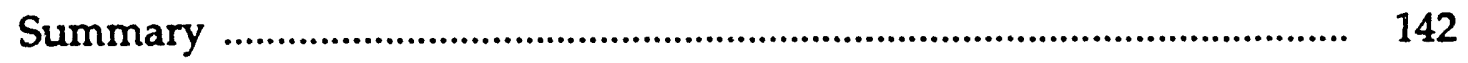

Conclusions and Future Directions ........................................................ 143

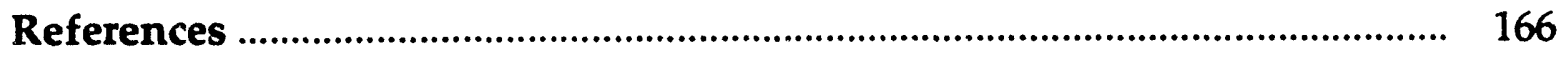




\section{List of Figures}

\section{Chapter 1}

Figure 1.1: Afternoon LADWP utility load vs. air temperature............... 12

\section{Chapter 2}

Figure 1.2: Ozone concentrations vs. air temperature ............................ 13

Figure 2.1: Urban energy balance ................................................................ 44

Figure 2.2: Rural energy balance................................................................ 45

Figure 2.3: Anthropogenic heating profiles................................................... 46

Figure 2.4: Urban radiative transfer ......................................................... 47

Figure 2.5: Atmospheric layers ............................................................... 48

Chapter 3

Figure 2.6: Combined BLM/CLM model ..................................................... 49

Figure 3.1: Two-dimensional modeling domain ........................................... 75

Figure 3.2: Diurnal profile for anthropogenic heat ..................................... 76

Figure 3.3: Base case wind speeds ............................................................... 77

Figure 3.4: Base case air temperatures .......................................................... 78

Figure 3.5: SNO case wind speeds............................................................ 79

Figure 3.6: SI case wind speeds................................................................. 80

Figure 3.7: LI case wind speeds ............................................................... 81

Figure 3.8: Temperature profiles for localized cases.................................... 82

Chapter 4

Figure 4.1: Roughness averaging techniques............................................ 112

Figure 4.2: Sensible heat flux predictions ..................................................... 113

Figure 4.3: Latent heat flux predictions ....................................................... 114

Figure 4.4: Comparison of vegetation schemes ........................................... 115

Figure 4.5: Partitioning of anthropogenic heat ............................................. 116 Chapter 5

Figure 5.1: Computational and analysis domains ....................................... 147

Figure 5.2: Homogeneous case wind profiles ................................................ 148

Figure 5.3: Homogeneous case temperature contours ............................... 149

Figure 5.4: Homogeneous case temp. and PBL for specified cities.......... 150

Figure 5.5: Homogeneous case vertical cross-section ................................ 151

Figure 5.6: Heterogeneous case velocity and temperature comparison. 152

Figure 5.7: Heterogeneous case temp. and PBL for specified cities......... 153

Figure 5.8: Anthropogenic case velocity and temperature comparison. 154

Figure 5.9: Regions of albedo and vegetation modification....................... 155

Figure 5.10: Morning wind vector impacts of moderate cases.................. 156

Figure 5.11: Afternoon wind vector impacts of moderate cases ................ 157

Figure 5.12: PBL height impacts of moderate cases ...................................... 158

Figure 5.13: Morning temperature impacts of moderate cases .................. 159

Figure 5.14: Afternoon temperature impacts of moderate cases ............... 160 
Figure 5.15: Diurnal temperature depressions of moderate cases............. 161

Figure 5.16: Moderate case vertical cross-section.......................................... 162

Figure 5.17: Diurnal PBL height depression of extreme cases ................... 163

Figure 5.18: Diurnal temperature depression of extreme cases ................. 164

Figure 5.19: Combined case temperature impacts ........................................ 165 


\section{List of Tables}

\section{Chapter 2}

Table 2.1: Acceptable errors in flux calculations 21

Table 2.2: Comparison of various classes of climate models. Chapter 3

Table 3.1: Initialization sounding profiles 54

\section{Chapter 4}

Table 4.1: Land-use categories and surface characteristics. 86

Table 4.2: Larid-use categories and substrate characteristics

Table 4.3: Statistical model parameterization results 106

\section{Chapter 5}

Table 5.1: Initial sounding profiles ......................................................... 121

Table 5.2: Surface characteristics for homogeneous case ........................ 123

Table 5.3: Surface characteristics for heterogeneous case ...................... 127

Table 5.4: Surface characteristics for specific cities .................................. 128

Table 5.5: Albedo and veg. values before/after modification ................ 136 


\section{Acknowledgements}

This thesis is the culmination of many years of education and research. Along the way there were many individuals and organizations who were instrumental in my intellectual growth. As a graduate student I have been fortunate to be involved with several very successful research groups. First, I would like to thank my advisors, Professor Van Carey and Dr. Hashem Akbari for their mentorship. The Heat Island Project at Lawrence Berkeley Laboratory provided a wonderfuil atmosphere for conducting research. I am especially indebted to this group of researchers and friends; Professor Art Rosenfeld, Dr. Haider Taha, Dr. Kenneth Orvis, and Dr. Ronald Ritschard.

In addition to this group of researchers, I received support from many friends and family who gave me the encouragement I needed along the way. Above all, I would like to thank Karen for making this long road bearable, and for being a terrific partner in every important aspect of life. 


\begin{abstract}
Role of Surface Characteristics in Urban Metenrology and Air Quality

by

David Jean Sailor
\end{abstract}

\begin{abstract}
Doctor of Philosophy in Mechanical Engineering
University of California at Berkeley

Professor Van P. Carey, Chair
\end{abstract}

Urbanization results in a landscape with significantly modified surface characteristics. The lower values of reflectivity to solar radiation, surface moisture availability, and vegetative cover, along with the higher values of anthropo. genic heat release and surface roughness crmbine to result in higher air temperatures in urban areas relative to their rural counterparts. Through their role in the surface energy balance and surface exchange processes, these surface characteristics are capable of modifying the local meteorology. The impacts on wind speeds, air temperatures, and mixing heights are of particular importance, as they have significant implications in terms of urban energy use and air quality.

This research presents several major improvements to the meteorological modeling methodology for highly heterogeneous terrain. A land-use data base is implemented to provide accurate specification of surface characteristic variability in simulations of the Los Angeles Basin. Several vegetation parameterizations are developed and implemented, and a method for including anthropogenic heat 
release into the model physics is presented. These modeling advancements are then used in a series of three-dimensional simulations which were developed to investigate the potential meteorological impact of several mitigation strategies. Results indicate that application of moderate tree-planting and urban-lightening programs in Los Angeles may produce summertime air temperature reductions on the order of $4^{\circ} \mathrm{C}$ with a concomitant reduction in air pollution. The analysis also reveals several mechanisms whereby the application of these mitigation strategies may potentially increase pollutant concentrations. The pollution and energy use consequences are discussed in detail.

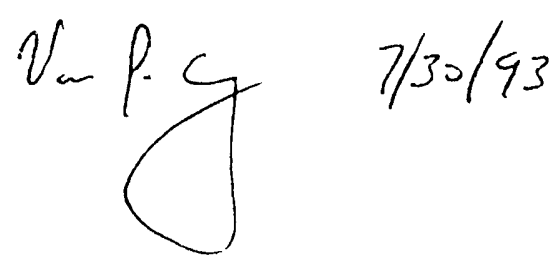




\section{CHAPTER 1 \\ Introduction to the Urban Climate}

The work contained within this dissertation focuses on improving the understanding of climatological and pollution effects related to urbanization through development and analysis of two-dimensional and three-dimensional meteorological simulations. These simulations have been designed to explore the impacts of the urban surface structure on meteorology with particular attention paid to air temperatures, wind speeds, and mixing depths. All meteorological effects are then related to potential impacts on urban energy use and air qualitv.

A necessary starting point for this dissertation is to provide the reader with a detailed description of the urban climate with a clear distinction between urban and non-urban climates. This short chapter serves this purpose with an emphasis on preparing the reader to appreciate the numerical simulations presented in the remainder of the text.

\section{HISTORICAL BACKGROUND}

It is well known that the climate of urban areas is generally quite different from the rural surrounds with elevated air temperatures and higher levels of air pollutants, including photochemical smog. Researchers have been investigating these phenomena for many years and have compiled thousands of publications which focus on urban climatology. From a historical perspective there are several contributors to the field of urban climatology who deserve to be mentioned here. 
Among the first to document the climatological impacts of urbanization was Luke Howard (1833) who investigated the temperature differences between urban London and its rural surrounds. From 1818 to 1833 Howard published several monographs dealing with the climate of the city. Howard, a chemist by training, was a pioneering meteorologist and the first to measure and document what later became known as the urban heat island.

Two decades later, a French scientist, Renou (1855) hypothesized that the polluted atmosphere of Paris was primarily responsible for the early evening temperature elevation within the city relative to the unpolluted rural surrounds. In his writings he colorfully describes Paris in terms of respiration of humans and animals and emissions from chimneys, and notes that these factors clearly must form the cause of the elevated urban temperature in Paris. Renou was one of the first to formally link anthropogenic and biogenic emissions to urban air temperatures and air quality. Today this link is still the subject of much active research.

The study of the urban climate continued over the next 50 years with a number of investigations into urban-rural climate differences. While Renou had postulated some causes, true systematic investigation of the causes of climate differences remained largely unexplored until early in the twentieth century. Wilhelm Schmidt (1927 and 1930) was the first to use instrumented motorized vehicles as a research tool to investigate the causes of urban rural temperature differences. His studies were the precursor to modern-day remotely-sensed evaluation of climate through use of high-precision radiometers mounted on aircraft and satellites.

Landsberg (1981) provides a fairly detailed history of the developments of urban climatology which have been summarized above. In addition to 
Landsberg's book, Atwater (1972), Chandler (1965), Changnon (1976), and Oke (1987) also provide cogent descriptions of the urban climate with discussions of the thermodynamic impacts of urbanization on the urban boundary layer. Akbari et al. (1988) and Bennet and Saab, (1982) extend these discussions to the impacts of urbanization on energy use and air quality.

In the past several decades, computers have become a powerful research tool in all areas of meteorology. Computational simulation of the urban climate is complicated by the high degree of heterogeneity in the urban surface. Improving upon the ability of models to accurately represent surface processes in heterogeneous regions has been the subject of numerous research efforts in the last several years. Two primary applications of such research are in the areas of General Circulation Modeling (GCM) and Urban Climatology. While the present work addresses issues in Urban Climatology, it also has implications for subgrid scale parameterizations in GCM studies where the problem of heterogeneous surface representation is simply on a larger length scale.

\section{THE URBAN FABRIC}

Urban areas are generally comprised of a complex combination of large and small structures and surface coverings woven together to make up what is referred to as the urban fabric. Among the most evident urban features are residential and commercial buildings, roadways, and parking lots. In the process of urbanization, these structures replace natural features such as bare soil and vegetation, creating a very different surface.

The differences in the urban fabric are manifested in te:ms of modifications in the basic surface and substrate characteristics. In terms of the energetics of the urban surface, the important characteristics are albedo, moisture availability, 
vegetative cover, and roughness length. The substrate characteristics of importance are substrate moisture content, thermal conductivity, thermal diffusivity, and density. Each of these parameters plays a role in the heat, moisture, and momentum transfer between the surface and the air which fiows above the surface.

\section{CHARACTERIZATION}

It is important to be able to characterize urban areas in terms of their surface and substrate characteristics. A typical characterization process consists of the following steps. First, a group of land-use categories is defined. Researchers generally disaggregate the urban fabric into anywhere from 4 to 20 categories. Examples from such a breakdown are: close-spaced residential housing, city core, industrial areas, parks, and forests. For a given region of the country, researchers can assign representative values of each of the surface characteristics to each land use category. Thus, the surface roughness of parks may be given the value of $0.20 \mathrm{~m}$ and the roughness of the city core may be assigned the significantly higher value of $1.50 \mathrm{~m}$. The second step in the characterization process is the acquisition and analysis of images from aircraft or satellites. These images along with various local maps can be used to obtain a distribution of land use within the city. If the city is then divided into grid cells for a computational simulation, the surface characteristics for each grid cell may be evaluated from knowledge of the land use distribution and the surface characteristic values associated with each land use. The scales of the land use data and computational model grid cells are generally quite different with nominal length scales of 0.1 $\mathrm{km}$ and $5.0 \mathrm{~km}$ respectively. For this reason, specification of grid cell characteristics from aggregation of land use data is not trivial. This issue will receive 
significant attention in the following chapters.

\section{URBAN HEAT ISLANDS}

Urban areas generally possess low values of albedo, vegetation cover, and moisture availability. These characteristics along with the existence of high levels of anthropogenic heating are generally associated with the phenomenon known as the urban heat island (UHI). That is, they explain why urban areas generaliy act as islands of elevated temperatures relative to the natural areas surrounding them. This phenomenon is known to be expanding in direct correlation to the rate of urbanization and population growth (Karl et al., 1988; Kukla et al., 1986;. The UHI is actually beneficial in the winter when the heat island effect results in less demand for residential and commercial heating. The summertime heat island (SHI), on the other hand, with an intensity often in excess of $2^{\circ}$ to $4^{\circ} \mathrm{C}$ in mid-latitude cities, results in an increase of up to $10 \%$ in the demand for air conditioning electricity (Akbari et al., 1990). This elevated load generally occurs in the late afternoon hours, corresponding to the peak summer electric utility load. The result is that utilities are forced to expand their use of the more expensive forms of electricity generation to meet the augmented peak load. Akbari et al. (1990) demonstrated the relationship between air temperature and electric utility load for southern California. They showed an increase in the 4 pm utility load of more than 2\% per degree Celsius (see Figure 1.1). This corresponds to more than $\$ 50,000$ per hour per degree Celsius in terms of energy cost for the L.A. basin! Temperature is also important in the photochemical reactions responsible for smog, typically measured in terms of ozone $\left(\mathrm{O}_{3}\right)$ concentration. The importance of temperature in the generation of photochemical smog is illustrated in Figure 1.2. This figure reveals a strong correlation between ozone 
concentration and air temperature. The dashed line in this figure indicates that in 1985 L.A. only exceeded the National Ambient Air Quality Standard (NAAQS) for ozone of 12 parts per hundred-million ( $\mathrm{pphm}$ ) on days for which the maximum temperature exceeded $22^{\circ} \mathrm{C}$. In addition, the concentration of ozone appears to increase by approximately $0.75 \mathrm{pphm}$ per degree $C$ increase in maximum air temperature. This correlation results from a number of factors including the temperature-dependence of the photochemical reaction mechanism.

\section{EMISSIONS}

In addition to surface modifications, urban-dwellers are responsible for large emissions of heat, moisture, and various pollutants into the atmosphere. Automobiles are a common example of urban pollution generators. As gasoline is consumed by an automotive engine, a large amount of heat is released. Also, these engines result in hydrocarbons, oxides of nitrogen, and other pollutants which issue out of the exhaust pipe. Another source of urban heat release is waste heat from electricity.

In addition to the anthropogenic emissions from vehicles, factories, commercial buildings, and residential buildings, the urban airshed must contend with emissions from nearby biogenic sources. Specifically, vegetation is responsible for humidifying the air through evapotranspiration, and polluting the environ-

ment through biogenic emission of hydrocarbons such as monoterpenes and isoprene. At the same time, vegetation acts as a sink for carbon dioxide which it uses in photosynthesis, a sink in the surface energy balance due to the latent heat transfer associated with evapotranspiration, and a sink for particulate pollution matter. While biogenic emissions are generally an order of magnitude smaller than the emissions from anthropogenic sources, their reactivity is generally much 
higher. In fact, the atmospheric lifetime for biogenically-emitted isoprene and monoterpenes is generally measured in hours, whereas the atmospheric lifetimes of automotive emissions such as benzene, toluene and propene are measured in days or months (Atkinson, 1988; Winer et al., 1992). The net result is that, in heavily vegetated regions, the emissions from biogenic and anthropogenic sources are equally important. Chameides et al. (1988) showed that in Atlanta, biogenic emissions alone were sufficiently high to cause predicted levels of ozone above the National Ambient Air Quality Standards limit of 12 pphm ozone.

Biogenic and anthropogenic emissions form the precursor base for the generation of photochemical smog. The rate at which smog is formed from its preclrsors is directly linked to air temperature via the rate constants for the numerous chemical reactions involved. It is also important to note that the biogenic emission rate for hydrocarbons is a positive function of temperature. For isoprene and the monoterpenes, there is roughly a $10 \%$ increase in biogenic emissions for a single degree Celsius increase in air temperature in the range of $30^{\circ} \mathrm{C}$ (Guenther, 1991). In the case of isoprene, Guenther showed that there is actually a peak emission at a temperature of roughly $35^{\circ} \mathrm{C}$ at which point, increasing temperature results in decreasing emissions. Hare then is another positive feedback mechanism between the SHI and the production of photochemical smog.

\section{PHOTOCHEMICAL SMOG}

The typical atmosphere is composed primarily of a handful of gases. Chief among these is molecular Nitrogen, $N_{2}$ which, at sea-level, occupies $78 \%$ of the atmosphere by volume. Oxygen, $\mathrm{O}_{2}$ is the next most abundant gas at roughly $21 \%$. These two fairly innocuous gases combine to account for $99 \%$ of the typical 
atmosphere at sea-level. Argon, $\mathrm{Ar}$ accounts for most of the remaining $1 \%$ of the atmosphere. It is the very small remaining fraction of additional gases which are responsible for the phenomenon known as photochemical smog. These gases along with photolysis reactions, produce ozone, $\mathrm{O}_{3}$ which is the standard measure of photochemical smog. The mechanism responsible for generating ozone is quite complex, but may be understood through a brief discussion of the chemical reactions which are most significant. The most important inorganic reactions in the ozone chain are as follows:

$$
\mathrm{NO}_{2}+h v \rightarrow \mathrm{NO}+\mathrm{O},
$$

where $h v$ is the energy associated with one photon of light. First, radiant energy (sunlight) breaks up nitrous oxide producing nitric oxide and releasing the oxygen radical. This oxygen radical may then become a part of any of several additional reactions, the most important being the combination with molecular oxygen to produce ozone,

$$
\mathrm{O}+\mathrm{O}_{2}+\mathrm{M} \rightarrow \mathrm{O}_{3}+\mathrm{M}
$$

where $\mathrm{M}$ is a catalyst such as $N_{2}$. As ozone is produced by (1.2), it reacts with NO breaking down into molecular oxygen again:

$$
\mathrm{O}_{3}+\mathrm{NO} \rightarrow \mathrm{NO}_{2}+\mathrm{O}_{2}
$$

A similar reaction occurs with $\mathrm{NO}_{2}$. These reactions are primarily responsible for the formation of $\mathrm{O}_{3}$. At the same time, however, other photolysis reactions such as the photolysis of nitrous acid are providing additional free radicals. Also of immerise importance in the photochemical reaction chain are reactions involving hydrocarbons, water vapor, and carbon monoxide. These additional reactions result in the production of additional radicals and they restock the supply of $\mathrm{NO}_{2}$ which is a key ingredient in the production of $\mathrm{O}_{3}$. 
Each of the chemical reactions discussed above occur at rates which are dependent upon concentrations of the reactants and given rate constants. In turn, many of the rate constants depend strongly on air temperature; they have high activation energy.

There are a number of important links, then, between urban meteorology and photochemical smog. First, temperature affects smog formation in two ways; the rate constants are heavily dependent upon temperature, and the emissions of pollutants have been shown to increase with temperature. This increase in anthropogenic and biogenic emissions will enhance the concentrations of photochemical precursors, resulting in higher levels of smog. Second, mixing height is intimately tied to precursor concentration levels. The higher the boundary layer, the more the surface emissions are diffused, resulting in lower concentrations and reduced smog levels.

\section{MITIGATION}

The urban climate is responsible for many negative effects. Human comfort is adversely affected through elevated temperatures, increased humidity, and higher levels of pollutants. Also, the demand for electricity for air conditioning has been shown to be strongly affected by air temperatures. In fact, the summertime peak electricity load for utilities in mid-latitude climates generally corresponds closely to the time of the peak air temperatures. The increase in electricity demand resulting from the SHI further increases urban air temperatures. In addition, the resulting power plant emissions further pollute the environment. One plausible benefit of the SHI in terms of pollution is that since the SHI generates a large-scale circulation, the aimospheric reactants and the existing photochemical smog are more thoroughly dispersed and ventilated away from the city. 
There are several methods by which human activities may counteract the effects of urtanization. While most cities are relatively devoid of vegetation, some urban areas are now implementing massive tree-planting programs in an attempt to beautify the city, cool the air, and sequester $\mathrm{CO}_{2}$ (McPherson, 1992). Benefits of tree planting programs have been evaluated in numerous studies (Akbari et al. 1992; Buffington, 1979; Laechelt and Williams 1976; Parker 1982; and Parker 1989). Another method which has yet to become as widely accepted is the notion of cooling the city by increasing the urban reflectivity to solar radiation, or albedo. In the first method, energy is converted into latent heat through evapotranspiration, while in the latter method an increased percentage of radiant energy is simply reflected from the urban surface back through the atmosphere and into space.

The potential benefits of SHI mitigation schemes need to be further evaluated. In general, these programs can be costly to initiate. While vegetation schemes may cost on the order of $\$ 10$ per tree to implement, the costs in terms of required maintenance and watering may be significantly higher. Albedo augmentation schemes, on the other hand, may be relatively cheap, since they may be implemented according to normal maintenance schedules with only a slight incremental cost. As an example, repaving with a lighter color aggregate or choosing a lighter color material for a scheduled re-roofing adds little or no cost. Much of the cost of these mitigation programs, therefore will be associated with management overhead. It is therefore important to be able to show that the savings of such programs will be worth the cost of implementation. While the direct effects of mitigation schemes will be reduced air temperatures and lowered levels of photochemical smog, the savings will ultimately need to be evaluated in terms of reduced energy consumption and reduced health risks. Preliminary 
estimates of such measures indicate that they are quite economical. Akbari et al. (1990) estimate that the cost of conserved energy to implement these schemes is between 0.2 and 1.0 cents per $\mathrm{kWh}$. This compares with a baseline consumer cost of 8 to 10 cents per $\mathrm{kWh}$ used.

In addition, there are other issues such as global warming trends which should be investigated in terms of their potential impact on urban comfort, energy use, and pollution. Finally, urban planners need to be able to predict the impacts of further urbanization including the production of factories and other emitters of anthropogenic heat, moisture, and pollution. For these reasons, meteorological modeling is both a useful and necessary tool in guiding urban policy.

As will be pointed out in this dissertation, the current state of urban meteorological modeling lacks the suphistication to deal with many of the problems just discussed. Improvements are needed in the area of urban surface characterizations, including the ability to model highly heterogeneous terrain, inclusion of anthropogenic heat release, and parameterization of partially vegetated domains. The results of the meteorological simula'ions presented in this dissertation form a basis for improved modeling of the urban climate. The goal of this research is to improve the understanding of the relationship between surface characteristics and the urban climate, and improve upon the ability to predict the effect of human activity on urban meteorology and air pollution. 


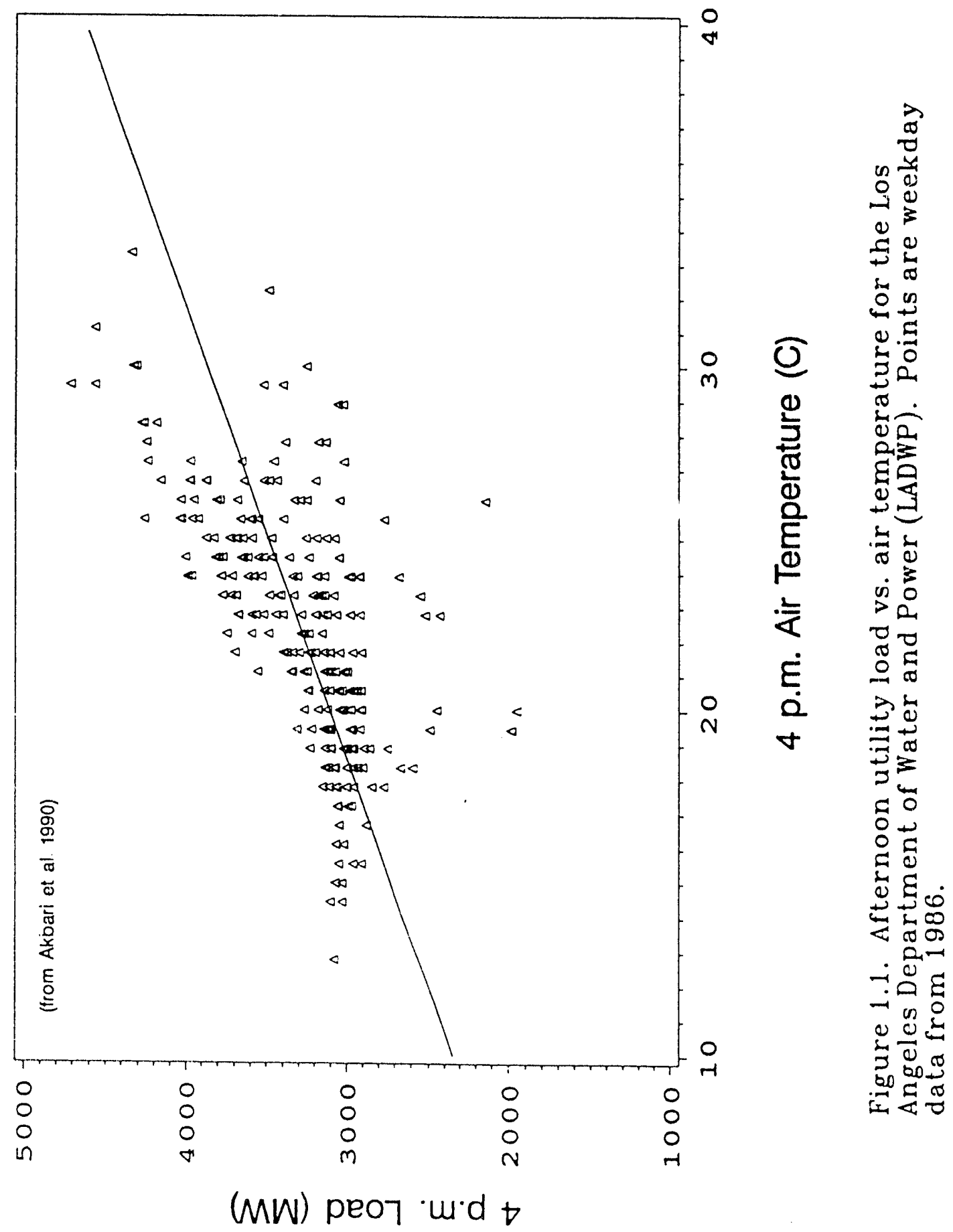




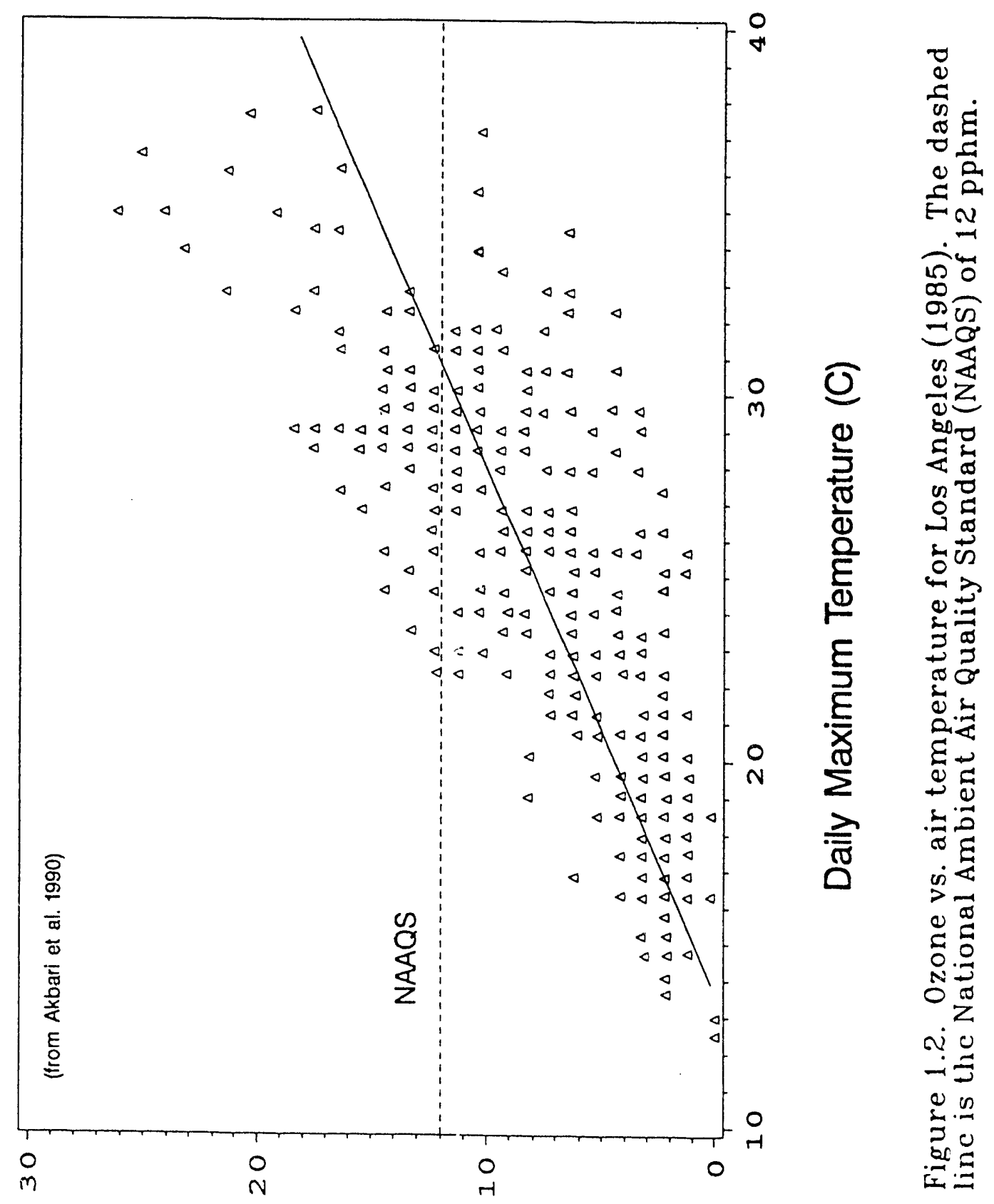

(WHdd) əuozo unu!xew K!!eo 


\section{CHAPTER 2 \\ Review of Urban Meteorological Modeling}

\section{URBAN ENERGY BALANCE}

In order to present a meaningful discussion of the problems associated with modeling urban climates, it is necessary to first present a short discussion of the urban energy balance and consider how the various urban surface parameters affect this balance. By describing each term in the energy balance equation and providing hypothetical but realistic estimates for its magnitude, one can qualitatively understand the importance of modeling the different aspects of the urban environment. The following discussion will focus on summertime mid-latitude urban and suburban climates.

\subsection{Energy equation}

For an individual surface such as a building wall or bare soil, the following simple energy balance applies at any instant in time:

$$
\alpha_{S} Q_{S}+\alpha_{L} Q_{L} \downarrow=Q_{L} \uparrow+Q_{H}+Q_{E}+Q_{G}
$$

where $\alpha_{S}$ and $\alpha_{L}$ are absorptivities for short- and long-wave radiation, $Q_{S}$ is the short-wave radiant flux, $Q_{L} \downarrow$ and $Q_{L} \uparrow$ are the downward and upward longwave radiative fluxes, $Q_{H}$ is the sensible heat flux, $Q_{E}$ is the latent heat flux, and $Q_{G}$ is conductive heat flux into the substrate (ground).

Each term of the energy balance depends on many factors and varies over the course of a given day. Typical summertime diurnal profiles of these energy 
flux terms are presented for an urban surface in Figure 2.1. The corresponding profiles for a rural surface are given in Figure 2.2. The data for these figures are taken from Oke (1987), and represent the average of 30 summertime days in 1983 for Vancouver B.C. $\left(49^{\circ} \mathrm{N}\right)$. In these figures heat flux toward the surface is shown as positive. Thus, evaporation is depicted as a negative flux. The net radiative flux is the largest term reaching $500 \mathrm{~W} \mathrm{~m}^{-2}$ at mid-day. This term represents the sum of the absorbed incident radiation less the emitted long-wave radiation. For the urban profiles, the sensible heat flux is the next largest term, followed by the latent flux. In the rural case of Figure 2.2, however, the latent heat flux is more significant than the sensible flux. These differences in flux profiles are clearly related to the difference in moisture availability and vegetative cover between urban and rural land uses. In addition, the level of anthropogenic heating in a rural area is shown to be essentially zero, whereas the anthropogenic heating term in urban areas can reach a significant level, often exceeding $50 \mathrm{~W} \mathrm{~m}^{-2}$ in the urban core. For suburban residential zones, which are of primary interest in this research, the corresponding level of anthropogenic heating is generally in the range of 1 to $10 \mathrm{~W} \mathrm{~m}^{-2}$. A detailed discussion of the energy terms is presented in the following sections.

\section{RADIATION}

Solar radiation incident on the earth's outer atmosphere is attenuated and scattered as it passes through the gases and particulates which make up the atmosphere. The "solar constant" is $1357 \mathrm{~W} \mathrm{~m}^{-2}$ above the atmosphere. After atmospheric attenuation, and accounting for solar zenith angle, the total summertime solar radiation reaching the mid-latitude surface of the earth at solar noon is generally less than $1100 \mathrm{~W} \mathrm{~m}^{-2}$. During the winter months and on overcast days, the peak daily solar insolation is obviously much smaller. The sum of 
the direct and diffuse solar radiation incident on a unit area surface is $Q_{S}$. Similarly, a given surface is also irradiated with long-wave radiation from the local surroundings and the sky, $Q_{L} \downarrow$. The surface also emits long-wave radiation as a function of its temperature, $Q_{L} \uparrow=\varepsilon \sigma T^{4}$. Computationally, this term is often solved as the residual when each other term in (2.1) is evaluated.

Reflectivity is the radiative characteristic which determines how much of this incident radiation is reflected by a surface. Reflectivity is a function of the surface involved and the wavelength of the incoming radiation. The total reflectivity of a surface integrated over the short-wavelength regime $(0.2 \mu \mathrm{m}$ to $4.0 \mu \mathrm{m}$ ) of special importance to meteorologists, is called the albedo, and will be referred to in this text by the symbol $\rho_{S}$, or equivalently $\left(1-\alpha_{S}\right)$.

$$
\rho_{S}=\frac{\int_{\lambda_{1}}^{\lambda_{2}} \rho(\lambda) d Q_{i}(\lambda)}{\int_{\lambda_{1}} d Q_{i}(\lambda)}
$$

where $Q_{i}(\lambda)$ is the incident hemispherical spectral energy intercepted by the surface, and $\rho(\lambda)$ is the hemispherical spectral reflectivity of the surface. The limits of integration $\left(\lambda_{1}\right.$ and $\left.\lambda_{2}\right)$ need to encompass the significant energy content portion of the solar spectrum. Generally, limits of 0.2 to $4.0 \mu \mathrm{m}$ are suitable.

Long-wave emitted radiation is associated with surface temperatures in the general range of $300 \mathrm{~K}$. Noting Wien's displacement law $(\lambda T)_{\max }=2898 \mu \mathrm{mK}$, this corresponds to wavelengths centered around $10 \mu \mathrm{m}$. The reflectivity of a surface with respect to long-wave radiation is typically assumed to follow the generalization of Kirchoff's law; $\alpha_{L}=\varepsilon_{L}$, where $\varepsilon_{L}$ is the emissivity of the surface at temperatures corresponding to long-wave radiative emission. For most surfaces, 
the absorptivity with respect to long-wave radiation is typically quite large, on the order of 0.95 to 0.98 . Metallic surfaces, however, often exhibit relatively low values of $\alpha_{L}$, on the order of 0.20 to 0.30 . As will be discussed later, the control of these radiative properties, $\rho_{S}$ and $\varepsilon_{L}$ presents a significant opportunity for urban planners to affect the surface energy balance.

\section{ANTHROPOGENIC HEATING}

Anthropogenic heating, historically referred to as furnace heat, is typically given the symbol $Q_{F}$. Some researchers include $Q_{F}$ in their representation of the surface energy balance (2.1). Although $Q_{F}$ is more appropriately included in a discussion of the volumetric energy balance of the near-surface air, it is briefly presented here for convenience. Some examples of anthropogenic heat sources are automobiles (and other combustion sources), appliances, air conditioners, and power plants. This is a difficult term to model accurately since it is dependent upon daily human activities that vary on diurnal, seasonal, and cultural schedules. While diurnal and seasonal variations of anthropogenic release activities may be expected to follow periodic and predictable patterns, the effect of cultural and societal factors is difficult to predict. Consider, for instance, the impacts that holidays and weekends have on energy consumption schedules. One solution to such difficulties is to present very generalized profiles which are representative of typical scenarios. Anthropogenic heat release schedules for urban and residential sites have been developed by Taha et al. (1992). Figure 2.3 illustrates the relative importance that $Q_{F}$ can have in the urban energy balance. This figure contrasts the summertime anthropogenic heat release for an urban area with that for a rural area. Whereas $Q_{f}$ is insignificant in rural areas, it can play an important role in the urban energy balance, where it may have a peak 
value as high as $50 \mathrm{~W} \mathrm{~m}^{-2}$ for the urban core. Depending upon the city and time of year the peak value may be much larger.

\section{SENSIBLE HEAT FLUX}

Convection is the process whereby heat is conducted from a surface to the adjacent air molecules and then diffused and advected away from the surface. The instantaneous magnitude of convective heat loss (or gain) is called the sensible heat flux, $Q_{H}$. The magnitude of this term depends upon wind velocities, the air-surface temperature difference, and surface roughness characteristics. The typical parameterization is given as:

$$
Q_{H}=\rho C_{p} U^{*} \Theta^{*}
$$

Here, $\rho$ and $C_{p}$ are the density and specific heat capacity of air; $U^{*}$ is the friction velocity, a function of wind speeds and surface roughness; and $\Theta^{*}$ is related to the driving temperature difference between the surface and the air. In the daytime, when the surface is warmer than the adjacent air, this heat flux is away from the surface (positive). At night, the sign of this term is generally negative because of the high rate of surface radiative cooling.

\section{LATENT HEAT FLUX}

The energy required to evaporate water is known as the latent heat of evaporation. Numericaliy it has the value of 2260 Joules per gram of water. The energy balance of a moist surface differs from that of a dry surface in that the latent heat term is significant. Vegetation also serves as a vehicle for latent heat transfer, acting as a pump, extracting subsurface moisture via the roots and transpiring this moisture via the leaves. The combined effect of evaporation and transpiration is known as evapotranspiration. While evapotranspiration yields a 
negative latent heating term, surface condensation results in a positive value. Meteorologists refer to the ratio of sensible to latent heat flux as the Bowen Ratio.

Latent heat flux from a bare surface is generally parameterized in a similar fashion to the sensible flux term:

$$
Q_{E}=\rho L_{e} U^{*} Q^{*}
$$

Here, $Q^{*}$ is related to the driving humidity difference between the air adjoining the surface and the first modeled layer of air, and $L_{e}$ is the latent heat of evaporization for water.

\section{SURFACE HEAT FLUX}

The incident radiant energy which is not partitioned into sensible or latent heat flux, or reradiated as long-wave flux, enters the surface as a conductive surface or ground flux, $Q_{G}$. During the day this flux is typically into the substrate which stores the energy according to its thermal capacity. At night, this stored energy is released through convection and long-wave radiation. This flux when integrated over a diurnal period is approximately zero. Models typically solve for the surface heat flux by evaluating the substrate temperature profile based on the one-dimensional heat diffusion equation subject to time-varying Dirichlet boundary conditions:

$$
\frac{\partial T}{\partial t}=\frac{k}{\rho C_{p}} \frac{\partial^{2} T}{\partial Z^{2}}
$$

\subsection{Accuracy of modeling terms}

Each of the energy flux terms just described plays an important role in the urban surface energy balance. This energy balance determines the surface temperature which in turn is intimately related to air temperature. In general these 
terms are highly interdependent and are modeled by a complex system of equations.

In order to obtain a qualitative understanding of the importance of accurately modeling each term in the surface energy balance, consider a typical midlatitude city at noon on a hot summer day. Suppose that the relative contributions of each of the energy terms are exactly as given in Table 2.1. Consider the requirement that we wish to model this energy balance accurately enough to predict surface temperature to within one degree Celsius. By rearranging (2.1) and noting that the outgoing long-wave radiative flux is given by $Q_{L} \uparrow=\varepsilon_{L} \sigma T_{g}^{4}$, one may solve for the surface temperature as:

$$
T_{g}=\left[\frac{Q_{L} \uparrow}{\varepsilon_{L} \sigma}\right]^{0.25}=\left[\frac{\alpha_{S} Q_{S}+\alpha_{L} Q_{L} \downarrow-Q_{E}-Q_{H}-Q_{G}}{\varepsilon_{L} \sigma}\right]^{0.25}
$$

For the hypothetical energy balances in Table 2.1 the computed surface temperatures are $26^{\circ}$ and $34^{\circ} \mathrm{C}$ for the rural and urban cases, respectively. This table presents the allowable percent errors in the parameterization of each energy term which by itself would result in a one-degree error in the instantaneous calculation of the surface temperature. These values are calculated from a straightforward differentiation of (2.6). For surface temperatures on the order of $30^{\circ} \mathrm{C}$ and surface emissivities around $0.95, Q_{L} \uparrow$ is $450 \mathrm{~W} / \mathrm{m}^{2}$. A one degree change in surface temperature corresponds to roughly a $6 \mathrm{~W} / \mathrm{m}^{2}$ change in $Q_{L} \uparrow$. Thus, an error of $6 \mathrm{~W} / \mathrm{m}^{2}$ in calculating any energy term in (2.2) will result in a surface temperature error of roughly $1^{\circ} \mathrm{C}$. 
Table 2.1: Acceptable Errors for $1^{\circ} \mathrm{C}$ Change in Calculated Ground Temperature

\begin{tabular}{|l||cc|cc|}
\hline $\begin{array}{c}\text { Term in } \\
\text { Eqn. 1 }\end{array}$ & Rural $^{\dagger}$ & $\begin{array}{c}\text { Acceptable } \\
\text { Error (\%) }\end{array}$ & Urban $^{\dagger}$ & $\begin{array}{c}\text { Acceptable } \\
\text { Error (\%) }\end{array}$ \\
\hline \hline$Q_{S}\left(W / m^{2}\right)$ & 800 & 0.9 & 760 & 1.0 \\
$Q_{L} \downarrow\left(W / m^{2}\right)$ & 350 & 1.8 & 365 & 1.8 \\
$Q_{E}\left(W / m^{2}\right)$ & 305 & 2.0 & 158 & 4.0 \\
$Q_{H}\left(W / m^{2}\right)$ & 150 & 4.0 & 240 & 2.6 \\
$Q_{F}\left(W / m^{2}\right)$ & 0 & - & 30 & 20 \\
$Q_{G}\left(W / m^{2}\right)$ & 80 & 7.5 & 148 & 4.2 \\
$\alpha_{S}$ & 0.20 & 0.9 & 0.14 & 0.9 \\
$\varepsilon$ or $\left(\alpha_{L}\right)$ & 0.98 & 1.3 & 0.98 & 1.3 \\
\hline$Q_{L} \uparrow\left(W / m^{2}\right)$ & 445 & -- & 495 & -- \\
\hline
\end{tabular}

t Energy balance cases are hypothetical, based partially on figures presented by Oke (1987).

Clearly, the larger the energy flux term, the more accuracy is required in modeling it. Thus, the first step toward accurately modeling an urban climate is the accurate calculation of the largest energy term, the incoming solar radiation. Given the time of day, time of year, and latitude, the short-wave energy incident on the earth's outer atmosphere is relatively simple to compute with accuracy. The most difficult problem in evaluating the short-wave radiation term involves knowing the extent of atmospheric attenuation and scattering, which in turn requires accurate knowledge of the atmospheric profiles of pollutant concentrations and water vapor as well as cloud cover. The accurate evaluation of the surface absorptivity of this short-wave radiation, $\alpha_{S}$ is also important. The modeling of latent, sensible, and incoming long-wave heat fluxes are next in order of importance. In cases of moist or heavily vegetated surfaces, however, the latent heat flux term may be of primary importance. The modeling accuracy of the surface flux and anthropogenic heat flux is seen to be of secondary importance. It should be pointed out that the above analysis neglects the interdependence of the 
energy flux terms. In reality, if one term is consistently underestimated, the other energy terms will adjust over the course of the day and the resulting error in surface temperature prediction may be smaller than predicted by the above analysis.

\subsection{The Urban Fabric (albedo and vegetation)}

Each of the energy terms is strongly influenced by the thermal, radiative, and geometric characteristics of the urban surface. What follows is a discussion of these characteristics and the potential to modify them through urban planning and large scale implementation of heat island mitigation measures. Specifically, the focus is on albedo and vegetative cover of urban surfaces.

The urban fabric itself is a source of great variability of albedo, and thus has high potential for significant albedo modification. A parking lot paved with standard asphalt, for instance, may have an albedo as low as $0.05\left(\alpha_{S}=0.95\right)$, whereas a concrete sidewalk will have an albedo of roughly $0.30\left(\alpha_{S}=0.70\right)$. Surfaces that are visibly light in color tend to have higher albedos, because the energy content of the visible spectrum is about half of the total energy content of the solar spectrum. So, use of light-colored surfaces and paints can significantly increase the albedo of a building or entire urban region. Researchers have predicted that moderate increases in the overall albedo of a city can significantly reduce urban temperatures, and consequently can lower consumption of energy and reduce air pollution. Specifically, Taha et al. (1988) using one-dimensional climate models estimated that increasing Sacramento's albedo from 0.25 to 0.40 would be possible and could lower peak urban summertime temperatures by as much as $4^{\circ} \mathrm{C}$. Recent measurements in White Sands New Mexico have indicated a similar relationship between naturally occurring albedo variations and measured ambient 
air temperatures (Rosenfeld et al. 1993).

In estimating albedo, it is important to note its variation as a function of solar zenith angle. Furthermore, the effective albedo of rough surfaces (i.e. an urban or suburban neighborhood) depends upon the view factors of the various individual surfaces. As an example, consider a simplified city made up of high albedo buildings and low albedo streets. The effective albedo is essentially an area-averaged albedo when the zenith angle is nearly zero (ie. the sun is directly overhead). As the zenith angle increases away from zero the albedo of the building surfaces plays an increasingly important role in determining the effective albedo. In addition, multiple reflections of incident radiation are common in cities where energy reflected by one surface may be intercepted and reflected by several additional surfaces before escaping the urban canopy. This effect is demonstrated in Figure 2.4. Consider the case where the albedo of each surface in this figure is 0.20 . For Ray 1 and Ray 3, each of which undergo a single reflection, the effective albedo is simply the albedo of the individual surface $(0.20)$. In the case of Ray 2, however, the effective albedo is $0.20 \times 0.20 \times 0.20$, or 0.008 . Clearly, then, the geometric roughness and surface to surface radiation view factors of a city result in an effective albedo which is less than the simple area-averaged albedo of individual surfaces. In addition, it is clear that this effective albedo is also a function of the solar zenith angle. This example illustrates the importance of taking the geometry into account when assessing the radiative balance of a city.

There are two primary methods of specifying surface albedo in urban meteorological studies. Often, researchers will obtain land-use data, assign a given albedo to each category of land use, and simply compute the appropriate area-average. In this case, the potential for multiple reflection and absorption of 
radiant energy is not accounted for and the resulting predicted albedo values may be artificially high. In the preferred situation, albedo values are derived from satellite or aircraft radiometry. Since this method involves actual measurement of reflected radiation, it can be expected to produce more realistic estimates of albedo for urban areas. At the same time, however, remotely-sensed estimation of surface albedo requires a detailed understanding of the radiative characteristics of the intervening atmosphere and is therefore subject to atmospheric correction errors.

Many researchers have targeted urban albedo as an important variable through which the urban microclimate may be selectively altered to reduce the magnitude of the impacts of the urban heat island. A number of studies have used computer models to simulate the change in building energy use resulting from changes in exterior building color (Griggs et al. , 1989; Conner, 1985; Reagan and Acklam, 1979). Results from the simulaiiuns of Taha et al. (1988), for instance, estimate a $0.6 \%$ and $0.4 \%$ savings in cooling energy per 0.01 increment in albedo for a poorly insulated house and a well insulated house, respectively. These studies, however, generally focus on the ability of localized albedo modifications to affect the surface energy balance of a building wall or roof. This is referred to as the "direct" effect of albedo modification. There is another impact which may be of equal significance. Air temperatures are linked to the surface temperature through convective heat transfer (sensible heating) and through the absorption of long-wave radiation by several gascous constituents in the atmosphere: namely, water vapor and carbon dioxide- the grienhouse gases. Thus, the local modification of albedo may indirectly result in a city-wide air temperature impact. 
Another urban design feature which has significance from a climatological perspective is vegetation. First, shading by trees has a direct impact on the building energy balance. In addition, through evapotranspiration, vegetation is able to dissipate much of the incident energy as latent heat. This effect not only has a direct effect on the air adjacent to the vegetation, but can have an indirect impact on buildings throughout the city as the cooled air is advected by local winds. The effect of a single tree at distances far removed from its location is difficult to quantify. Analysis of such indirect effects will be important, however, in the determination $o_{2}^{f}$ the effectiveness of heat island mitigation measures.

\section{CURRENT STATE OF URBAN CLIMATE MODELS}

Many investigators have used mathematical models to analyze the effects of urbanization on climate. Several comprehensive reviews of such models are available (Pielke, 1984; Oke, 1974, 1979). Pielke's book is useful as it not only gives background information on general mesoscale modeling, but also contains an appendix with detailed information on existing climate models. Oke (1979) reviewed a great number of these models and introduced two classifications which distinguish the models based on the scale of their vertical domain. Oke defined "canopy-layer models" as those models which focus on that portion of the city that is roughly below the building roof height. Models which focus primarily on the region of the atmosphere above building roof height, but within the first couple of kilometers from the surface where the effects of the surface friction are felt, are called "boundary-layer models." An additional category, "surface-layer models," is sometimes used when discussing urban climate modeling. Surface-layer models do not resolve individual buildings, nor do they extend to the top of the domain of influence of the surface. Instead, they 
approximate transfer processes in the surface layer where surface frictional forces dominate the flow regime and where turbulent fluxes of energy are roughly constant with height. Many boundary-layer models contain surfacelayer models within them to form a lower boundary condition. The distinction between surface-layer and boundary-layer models is that the latter predict atmospheric variables above the surface-layer as well as within it.

The four basic layers of interest to urban climate modelers are graphically depicted in Figure 2.5. The substrate is typically modeled as a thin layer on the order of a meter thick. When it is included in a model, substrate temperature and moisture content are calculated by numerical solution of the diffusion equations. The canopy layer extends to the top of the urban roughness elements. This is the most difficult layer to parameterize as it includes a very complex arrangement of geometric, radiative, and thermal factors. The surface layer, extending up to roughly 100 meters is the layer in which the effects of the surface roughness are directly manifested through turbulent interactions. Above the surface layer is the mixed layer which extends 1000 meters upward. In this layer the atmospheric variables are well-mixed.

Each class of models has applications in urban climate modeling, but each also has serious limitations. The goal of the following discussion is to provide background on the current state of these models and to point out the strengths, limitations, and applications of each as it applies to studies of the urban climate. For a more detailed discussion of these classifications, and for additional examples of specific models the reader is referred to Pielke (1984), Bornstein (1984, $1989)$ and Oke $(1974,1979)$. A summary of the key points of the present discussion is provided in Table 2.2. 
Table 2.2: Comparison of the Various Classes of Climate Models

\begin{tabular}{|l||l|}
\hline \multicolumn{1}{|c||}{ FEATURES } & \multicolumn{1}{|c|}{ Canopy-Layer Models } \\
\hline Domain & $\begin{array}{l}\text { Urban canyon extending from substrate to building } \\
\text { height, sometimes extending up to 10 building } \\
\text { heights. }\end{array}$ \\
\hline Equations Modeled & $\begin{array}{l}\text { Energy balance of urban surfaces. Occasionally the } \\
\text { momentum energy humidity and scalar transport } \\
\text { equations are solved on a finite difference grid } \\
\text { (Sievers 1986 for example). }\end{array}$ \\
\hline Input & $\begin{array}{l}\text { Typical input variables include month, latitude, } \\
\text { elevation, hourly profiles of temperature, relative } \\
\text { humidity, wind speed, pressure, atmospheric dust, } \\
\text { and canyon geometry. Some models incorporate } \\
\text { anthropogenic heat schedules. Typically atmos- } \\
\text { pheric variables at the top of the canyon are } \\
\text { required. }\end{array}$ \\
\hline Output & $\begin{array}{l}\text { Surface energy fluxes and surface temperatures. } \\
\text { For models which solve the equations of motion } \\
\text { (Sievers 1986) the atmospheric variables at grid }\end{array}$ \\
locations are provided.
\end{tabular}


Table 2.2: Continued

\begin{tabular}{|l||l|}
\hline \multicolumn{1}{|c||}{ FEATURES } & \multicolumn{1}{|c|}{ Surface-Layer Models } \\
\hline Domain & $\begin{array}{l}\text { Substrate up to top of surface layer, typically on } \\
\text { the order of } 100 \text { meters. }\end{array}$ \\
\hline Equations Modeled & $\begin{array}{l}\text { Energy balance at effective surface, and typically } \\
\text { the momentum energy humidity and scalar tran- } \\
\text { sport equations are solved in 1,2, or 3-D formula- } \\
\text { tions. }\end{array}$ \\
\hline Input & $\begin{array}{l}\text { Typical input variables include month, latitude, } \\
\text { atmospheric variables at the top of the model, sub- } \\
\text { strate thermal properties, surface roughness, } \\
\text { albedo, anthropogenic heat, and plant canopy } \\
\text { input. }\end{array}$ \\
\hline Output & $\begin{array}{l}\text { Temperature and energy fluxes at effective surface, } \\
\text { temperature profile within the substrate, and } \\
\text { profile of atmospheric variables at grid locations. } \\
\text { Vertical spacing between grid points in the lower } \\
\text { portion of the atmosphere is typically between 10 } \\
\text { and 50 meters, and time steps are on the order of } \\
\text { several minutes. }\end{array}$ \\
\hline Remarks & $\begin{array}{l}\text { Grid spacings are large, requiring averaging of sur- } \\
\text { face parameters and atmospheric variables. Com- } \\
\text { putational speed limits the resolution and duration } \\
\text { of 3-D schemes. }\end{array}$ \\
\hline
\end{tabular}


Table 2.2: Concluded

\begin{tabular}{|l||l|}
\hline \multicolumn{1}{|c||}{ FEATURES } & \multicolumn{1}{c|}{ Boundary-Layer Models } \\
\hline Domain & $\begin{array}{l}\text { Substrate up to top of mixed layer. Model top is } \\
\text { often variable, sometimes extending up to several } \\
\text { kilometers. }\end{array}$ \\
\hline Equations Modeled & $\begin{array}{l}\text { In addition to the standard transport equations, a } \\
\text { predictor for mixing height is often incorporated. }\end{array}$ \\
\hline Input & Similar to surface-layer model input. \\
\hline Output & Similar to surface-layer model input. \\
\hline Remarks & $\begin{array}{l}\text { Due to large grid spacing the details of the urban } \\
\text { surface are lost. As with the surface-layer models, } \\
\text { computational speed limits the resolution and } \\
\text { duration of 3-D schemes. }\end{array}$ \\
\hline
\end{tabular}

\subsection{Canopy-Layer Models}

Canopy-layer models (CLM's) describe phenomena that exist below the roof height, although they sometimes extend to several building heights. The study of the urban canyon is a prime example of the application of a canopy-layer model. Most CLM's do not predict ambient atmospheric variables. Instead, they typically use a set of energy-balance equations similar to (2.1) and (2.2) for various surfaces within the canopy to predict surface temperatures and surface fluxes. Bulk atmospheric properties, such as air temperature and wind speeds are often assumed or provided by field measurements. The energy-balance equations include net short-wave and long-wave radiation inputs to the surface and, in some cases, an input contribution from anthropogenic heat sources. Heat is 
dissipated by long-wave radiation, conduction to buildings and street surfaces, and turbulent diffusion of sensible and latent heat.

A few CLM's do predict ambient variables, using finite-difference solutions to fluid transport equations for a turbulent atmosphere. The equations solved generally involve a series of approximations to and averaging of the following transport equations for mass, momentum, energy, and specific humidity.

The conservation of mass equation is given by

$$
\frac{\partial u_{j}}{\partial x_{j}}=0
$$

where $u_{j}$ is the velocity in the $x_{j}$ direction. Variations in density have been ignored in this equation. The three momentum equations $(i=1,2,3)$ are given by

$$
\frac{\partial u_{i}}{\partial t}+u_{j} \frac{\partial u_{i}}{\partial x_{j}}=-\frac{1}{\rho} \frac{\partial p}{\partial x_{i}}-g_{i}+\frac{\partial}{\partial x_{j}}\left(K_{M} \frac{\partial u_{i}}{\partial x_{j}}\right)
$$

where $p$ is pressure, $g_{i}$ is gravitational acceleration $\left(g_{i}=0\right.$ for $\mathrm{i}=1$ or 2$), \rho$ is density, and $K_{M}$ is the turbulent exchange coefficient for momentum. The Boussinesq approximation is often applied to the vertical momentum equation. This assumption states that the vertical pressure gradient is hydrostatic, and replaces the dependence of this equation on density variations with a dependence upon temperature variations. The energy equation is given by

$$
\frac{\partial \theta}{\partial t}+u_{j} \frac{\partial \theta}{\partial x_{j}}=\frac{Q_{\theta}}{\rho_{o} C_{p}}+\frac{\partial}{\partial x_{j}}\left(K_{H} \frac{\partial \theta}{\partial x_{j}}\right)
$$

where $\theta$ is temperature, $Q_{\theta}$ is the energy source term, $C_{p}$ is constant pressure specific heat, and $K_{H}$ is the thermal conductivity. The specific humidity obeys an 
equation similar in form to the energy equation:

$$
\frac{\partial q}{\partial t}+u_{j} \frac{\partial q}{\partial x_{j}}=\frac{Q_{q}}{\rho_{o}}+\frac{\partial}{\partial x_{j}}\left[K_{V} \frac{\partial q}{\partial x_{j}}\right]
$$

where $q$ is the specific humidity, $Q_{q}$ is the vapor source term, and $K_{V}$ is the diffusion coefficient for vapor.

Additional scalar equations are sometimes specified to describe the transport of various pollutants. In fact, one popular use of CLM's is for the analysis of pollution dispersion in and around urban canyons. Models that solve the transport equaíions rely on specified boundary conditions to link them with conditions above the canopy. Existing CLM's do not interactively link the canopy to the rest of the planetar' boundary layer. In general CLM's require boundary conditions which are determined at the local and mesoscale level. Thus even those models that predict atmospheric variables cannot simulate effects resulting from the local and mesoscale dispersion due to surface modifications made throughout a city.

Terjung and Louie (1974) modeled urban absorption of short-wave and long-wave radiation and the dissipation of that input via re-radiation, conduction, convection, and evaporation. Their model was the first detailed threedimensional CLM. Given temporal, geographical, and climatic input data, the Terjung model predicts the following hourly output for streets, walls, rooftops, and lawns: absorbed diffuse, direct, and terrain-reflected short-wave radiation; total incident and emitted long-wave radiation; convection; conduction; evaporation; and surface temperatures. Anthropogenic heating is not included. The model also fails to predict air temperatures, humidity, or wind speed, and therefore, disallows feedback between surface heating and air temperature. 
Terjung and O'Rourke (1980) described a similar model, which has the added capability of modeling vegetated canopies. Their algorithm uses the heat balance equation $n f$ a leaf to construct an aggregated description of a multi-tiered canopy.

Arnfield (1982) presented a two-dimensional model for computing the net radiative parameters of an urban street canyon. In contrast to the model of Terjung and Louie, the Arnfield model can be used to investigate the effects of spatial variations in building materials because it allows radiative parameters of individual surfacess to be varied explicitly.

Sievers and Zdunkowski (1986) presented a model that predicts ambient variables in a two-dimensional urban canyon. The top of the model is well above the canopy layer, roughly at ten building heights. This model uses a streamfunction vorticity method to solve the momentum equations and predicts turbulent exchange coefficients with a mixing length formula. The energy budget of the canopy is described by a heat transport equation that accounts for anthropogenic sources.

In studying the urban heat island and its effect on building energy use, it is the urban canopy which is of primary importance. Specifically, evaluation of temperature, humidity, and the wind field in the lowest ten meters is necessary. In this respect, CLM's appear to be useful in the study of heat islands. The principal drawback of CLM's, however, is that since their vertical extent terminates at several building heights, accurate ambient boundary conditions must be specified at this height. In the past, researchers have used local climate data to provide these boundary conditions as a function of time of day. The conditions within the canopy are then determined as a function of specified upper boundary conditions. This scheme is satisfactory if the upper boundary is known. In heat 
island studies, however, the ambient variables above the canopy, being linked to the surface characteristics, will change as the surface characteristics are altered. Thus, the fact that CLM's do not allow feedback between the urban canopy and the upper boundary layer seriously limits their applications to heat island studies.

Another difficulty in applying CLM's is the need to accurately specify the surface properties, especially the surface roughness. Surface roughness is intimately involved in determining atmospheric turbulence and local wind profiles in the vertical domain of interest. Surface features can be responsible for significant changes not only in wind speed, but also in wind direction through the effects of local channeling. Using a CLM to model the climate over an entire city requires detailed specification of urban geometry. This can be a very tedious task, especially in studies of large urban areas.

\subsection{Surface-Layer Models}

Above the canopy layer, where flow is strongly influenced by the geometry of surface features, there is another region called the turbulent surface layer, or surface boundary layer. Most surface-layer models solve transport equations similar to equations (2.7a-d) in the layer extending from the surface to an upper boundary which typically ranges from 30 to 100 meters over an urban surface. As the horizontal extent of the domain becomes larger an additional term enters the horizontal momentum equation. This term is the Coriolis term which arises from the rotation of the earth. The horizontal momentum equations become:

$$
\frac{\partial u_{1}}{\partial t}+u_{j} \frac{\partial u_{1}}{\partial x_{j}}=-\frac{1}{\rho} \frac{\partial p}{\partial x_{1}}+\frac{\partial}{\partial x_{j}}\left(K_{M} \frac{\partial u_{1}}{\partial x_{j}}\right)+u_{2} f
$$

and 


$$
\frac{\partial u_{2}}{\partial t}+u_{j} \frac{\partial u_{2}}{\partial x_{j}}=-\frac{1}{\rho} \frac{\partial p}{\partial x_{2}}+\frac{\partial}{\partial x_{j}}\left[K_{M} \frac{\partial u_{2}}{\partial x_{j}}\right]-u_{1} f
$$

Equation (2.8a) is the east-west momentum equation, the "1-direction", and (2.8b) is the north-south or "2-direction" equation. In both equations, $f=2 \Omega \sin \phi$ is the Coriolis parameter, a function of the latitude, $\phi$. The rotational frequency of the earth is $\Omega=\frac{1}{24}$ hour $^{-1}$.

Outcalt $(1972 a, 1972 b)$ developed a one-dimensional surface-layer model (SLM), expanding primarily on the work of Myrup (1969) and Lettau (1969). The model is particularly notable for its simplicity, but retains elements typical of many SLM's. The model predicts surface heat fluxes and surface temperatures by iteratively solving a surface energy balance equation. It includes only one atmospheric layer, a turbulent surface layer, across which heat and moisture are assumed to turbulently diffuse. Within this layer, the turbulent heat fluxes are calculated using a formulation for the exchange coefficients appropriate for the surface layer in a neutral atmosphere. Outcalt added an empirical correction factor to account for the effect of non-neutral stability on the exchange coefficients.

\subsection{Boundary-Layer Models}

Boundary-layer models (BLM's) focus on the atmosphere above the canopy layer. Their vertical extent may be as high as several kilometers depending upon wind speed, surface roughness, and surface heating. Some BLM's extend to the top of the troposphere $(9-10 \mathrm{~km})$. Like SLM's, BLM's typically solve transport equations similar to (2.7) and (2.8) with various additional assumptions. In contrast to the CLM's, many existing BLM's provide dynamic feedback between the urban surface and the boundary layer. BLM's may also include predictions of 
the mixing height, which critically affects atmospheric variables in the boundary layer.

Carlson and Borland (1978) produced a one-dimensional BLM. This model is used primarily to simulate surface fluxes and surface temperature, but it also uses Monin-Obukhov similarity theory to estimate wind velocity, air temperature, and specific humidity at various heights. In the original model, surface fluxes were assumed to originate from a bare soil. More recently, however, Carlson et al . (1989) modified the model to include the effects of a vegetated surface. The model uses the Monteith resistance formulation (Monteith, 1975) to calculate the turbulent fluxes.

Bornstein et al. (1987) extended the two-dimensional vorticity-mode BLM of Bornstein (1975) to three dimensions by using a vector stream function. The model simulates time-varying, three-dimensional distributions of velocity, temperature, and sub-saturation moisture in the atmospheric boundary layer. The model uses the hydrostatic formulation which assumes horizontal length scales in the boundary layer are large compared to vertical length scales. This assumption simplifies the calculation of vertical velocities and reduces the amount of numerical computation, but also limits the horizontal resolution of the model. In Bornstein's three-dimensional model, 1.5 kilometers is about the minimum horizontal grid cell dimension. Anthropogenic heat flux is included in the model, and is allowed to vary spatially and on a diurnal cycle.

There are a number of operational BLMs which have been used for a widerange of applications. While these applications are too numerous to elucidate here, they include: evaluation of mountain and sea-breeze flows; the parameterization of mesoscale processes for inclusion in General Circulation Models (GCM); evaluation of global warming impacts on hydrology and vegetation; 
impact of deforestation on regional climate; and study of air pollution formation and transport. Two of the models which are currently seeing prominent usage are the Penn State/NCAR mesoscale model (PSUMM) and the Colorado State University Regional Atmospheric Modeling System (RAMS).

Surface-layer models and boundary-layer models share similar advantages and disadvantages with regard to modeling the urban climate. SLM's and BLM's both have the advantage over CLM's that they span a much larger domain, and allow feedback between the surface and the atmosphere. The largest drawback of these models is their difficulty in resolving surface characteristics. Since they have such a large domain, computational limitations require node spacings on the order of kilometers. In addition, the hydrostatic assumption applied in many of these primitive equation models further restricts the minimum node spacing. The hydrostatic assumption is generally assumed to be valid for horizontal grid spacings greater than 2 to $5 \mathrm{~km}$. Thus, the resulting output is really an integrated average over a volume of air on the order of a cubic kilometer.

\section{VALIDATION AND COMPARISON OF MODELS}

There are two primary methods of validating urban climate models. First, one can compare model predictions with existing climatological data. This is often difficult, as appropriate data are quite limited. Even when these data are available, essential concurrent information such as surface properties is often lacking. In addition, a model which performs well under one set of initial conditions may be prone to large errors when applied to a different geographic location, or under differing synoptic conditions. Two examples of massive climatological data collection experimerits are Metromex (Changnon, 1981) and the 
Stadtklima Bayern experiment (Brundl et al. 1985). In the Metromex experiment, data were collected for the St. Louis area in the early 1970's. The Stadtklima Bayern experiment consisted of continuous recordings of diurnal variations in temperature and humidity at 18 sites in the Munich area over a three and a half year period, beginning in July 1981. Eichorn et al. (1988) used the Munich data to validate their three-dimensional urban climate model. They found that temperature and humidity were well modeled in rural areas around Munich. When they compared model results for the downtown areas of Munich to measurements, however, they found modeled temperature profiles that deviated from the measured profiles by as much as $4^{\circ} \mathrm{C}$. Similarly, humidity calculations for downtown areas of Munich deviated from actual measurements by up to $15 \%$. Whereas the downtown temperature calculations were consistently higher than measured data, the relative humidity was overestimated in the daytime but underestimated in the mornings and evenings. It is likely that inadequacies in specification of surface characteristics were responsible for the discrepancies between model predictions and ambient measurements.

Although many models have proven useful in studying climate above homogeneous (rural) terrain, it is difficult to find an urban climate model that has proven itself to be accurate over urban canopies. Ross and Oke (1988) tested the performance of three urban energy balance models versus measurements for a suburb of Vancouver B.C. The models they investigated were those developed by Myrup (Myrup, 1969), Carlson (Carlson and Boland, 1978), and Atwater (Atwater, 1972). Ross and Oke were able to show that, although these models produced diurnal heat flux profiles which had the same characteristic shape as the measurements, they often deviated from the measurements by a substantial amount. They conclude that the inability of the models to handle the role of 
water availability and its impact on evapotranspiration is the principle problem. Based upon the earlier discussion of the urban energy balance, it should be noted that inaccuracy in evaluating this balance may, by itself, result in unacceptable errors.

A second method of validating climatological models is to compare them to existing well-documented and trusted models; models which have been previously tested against empirical data. Once a model has been applied to various meteorological situations and has compared favorably with measured data, it may thus serve as a benchmark for validation of other models. It should be noted, however, that each new application of a model may expose previously undiscovered weaknesses resulting from model assumptions or parameterizations.

\section{CLIMATE MODELS AND BUILDING ENERGY USE}

It has been mentioned that energy use is one area in which temperature change due to urbanization is of great importance. In particular, energy use in the buildings sector has been the focus of much recent work. In order to understand how local climate modification due to changes in urban characteristics influences building energy use researchers must first have a means of evaluating building energy use. Computer codes have been written that take as basic input building parameters, occupant schedules, and external climate variables, and that produce building energy use schedules. In addition to solar radiation data, the necessary climate variables include air temperature, specific humidity, wind speed, and wind direction, all evaluated within the height of the urban canopy. While climate can vary substantially within a city, and indeed from one side of a building to another, for present purposes it is desirable to predict volume 
averages at discrete steps in time. The average in the vertical dimension should be taken over the distance from the ground to roughly 10 meters. The scale for the horizontal averaging will depend on whether one is attempting a site analysis or an urban scale analysis. At the site scale an averaging area of 100 to 1000 square meters is appropriate since the nominal length scales of buildings are 10 to 50 meters. At the urban scale, however, the averaging area should be much larger, on the order of 10 square kilometers. Simulations of building energy also require a moderate degree of temporal analysis; hourly averages of climate variables are typically desirable.

\subsection{Previous studies}

Previous attempts to analyze building energy use and to quantify the impact of local climate change have been limited. Huang et al. (1987) and Taha et al. (1988) used the building energy program DOE-2.1C (DOE-2) to study the impact of heat islands on energy use. DCE-2 is a public-domain program that simulates the hourly energy performance of a building, incorporating information on its climate, type of building envelope, equipment use, and occupant schedules (U.S. Dept. of Commerce, 1980; Birdsall et al., 1985). For its climate input, DOE-2 uses hourly weather tapes that are available from a number of sources, including the U.S. National Oceanic and Atmospheric Administration (NOAA). The weather data, either from a local airport or from a Typical Meteorological Year (TMY) data tape for an airport at a nearby city, are taken to be representative of conditions around the modeled building.

In the studies of Huang et al. (1987) and Taha et al. (1988), the output data of a climate model were used in place of the standard airport data. This approach is simple and useful. The primary drawback of this method, however, is that 
since it ignores locally measured weather data it overlooks the possibility of discrepancies between the measured and simulated micro-meteorological variables.

This approach may be improved upon in the following way. First, base case urban landscape scenarios should be developed. A climate model is then used to predict diurnal temperature profiles. Similarly, test cases corresponding to albedo and vegetation modifications need to be developed and used in conjunction with a climate model to predict diurnal temperature profiles for these test cases. The hourly temperature differences between the base case and the test cases will then be calculated. Finally, the original weather data are modified by subtracting these temperature differences from the original temperature data. The modified tape data are then used in building energy studies to predict the impact of vegetation and albedo modifications.

This approach is useful since it makes the problem tractable. Unfortunately it also has several drawbacks which bring into question the accuracy of its predictions. First of all, it is clear that the climate at a nearby airport may not be representative of the climate within an urban center. It is also important to note that modifications to the weather data are made discretely. The resulting data tape, therefore, represents a compromise between measured and simulated data.

\subsection{A possible future approach}

Due to the above-mentioned constraints, it appears that modeling the effectiveness of heat island mitigation measures will require the development of new and very accurate urban climate models. These models will have to overcome the many obstacles pointed out previously. 
Rather than developing a completely new set of urban climate models it may be feasible to combine several existing models in such a way that their beneficial aspects are exploited. One of the more promising methods is the combining of existing CLM's and BLM's. A BLM could be given approximate areaaverages of urban surface variables as a lower boundary condition. Running the BLM for a 24-hour period would generate predictions of climate variables at distinct grid points. The analysis could then proceed by using this climate data at a specific height, say 50 meters, as an upper boundary condition to a canopy-layer model with a much finer grid. The CLM could take this information and a more detailed specification of the lower boundary urban surface, and be run for the same 24-hour period. The resulting climate information within the urban canopy would be a more accurate estimate than is currently available through application of a single model alone. A possible refinement could be obtained through the following iterative process. Since both the BLM and CLM would predict surface temperatures for the same 24-hour period, it is reasonable to compare the two surface temperature profiles. This would be accomplished by aggregating the fine grid CLM surface temperatures into representative averages for the BLM grid cells. If there is a significant difference between the surface temperature profiles, the BLM could be run again with a modified lower boundary condition. This modification could consist of an alteration of the integrated average of surface properties used by the BLM as a lower boundary condition to make the surface temperature results from the two models more consistent. Another, more straightforward approach would be to simply impose the resulting CLM surface temperature field as a lower boundary condition for the BLM. The BLM could be run a second time and the predicted $50 \mathrm{~m}$ meteorological variables would once again be used as upper boundary conditions for an improved CLM run. This 
process would then be repeated until the predicted surface temperature fields converge. A flowchart of the above proposal is given in Figure 2.6.

The results of such a inethod should be a fairly accurate representation of the climate within the urban canopy. As described earlier, these climate variables can then be used by another computer code such as DOE-2 to predict building energy use. This method may also be useful in high-resolution evaluation of the impacts of urban mitigation measures. After an urban site has been chosen for study, a comprehensive set of mitigation scenarios could then be developed and analyzed. Because of the great variability among cities, this analysis will be required for any urban area considering heat island mitigation measures. The results from such studies will be instrumental in determining urban energy policy.

\section{SUMMARY}

The current state of urban climate modeling is only marginally useful for application to urban heat island studies. Simple calculations show that the parameterizations of the urban energy balance terms must be extremely accurate in order to get reasonable predictions of ambient variables. The complex interactions of the urban surface parameters with the urban microclimate make simple estimation techniques unreliable. An effective way to get a measure of the effectiveness of urban heat island mitigation measures is to develop rigorous and accurate parameterizations for the urban fabric which in turn are used as boundary conditions for high resolution or nested numerical models. Specifically, the combination of a canopy-layer model and a boundary-layer model is suggested as providing great potential for success in the attempt to accurately model the urban climate. 
Clearly, there is much work to be completed before accurate simulations of urban climates can be accomplished and applied to the study of the impact of heat island mitigation measures on urban energy use and air quality. At this point, the capabilities of the numerical models appear to be satisfactory. It is the parameterizations of the urban surface and the specification of boundary conditions that are insufficient. Unique approaches such as the iterative scheme outlined above must be thoroughly investigated, so that accurate prediction of urban temperatures becomes possible. 


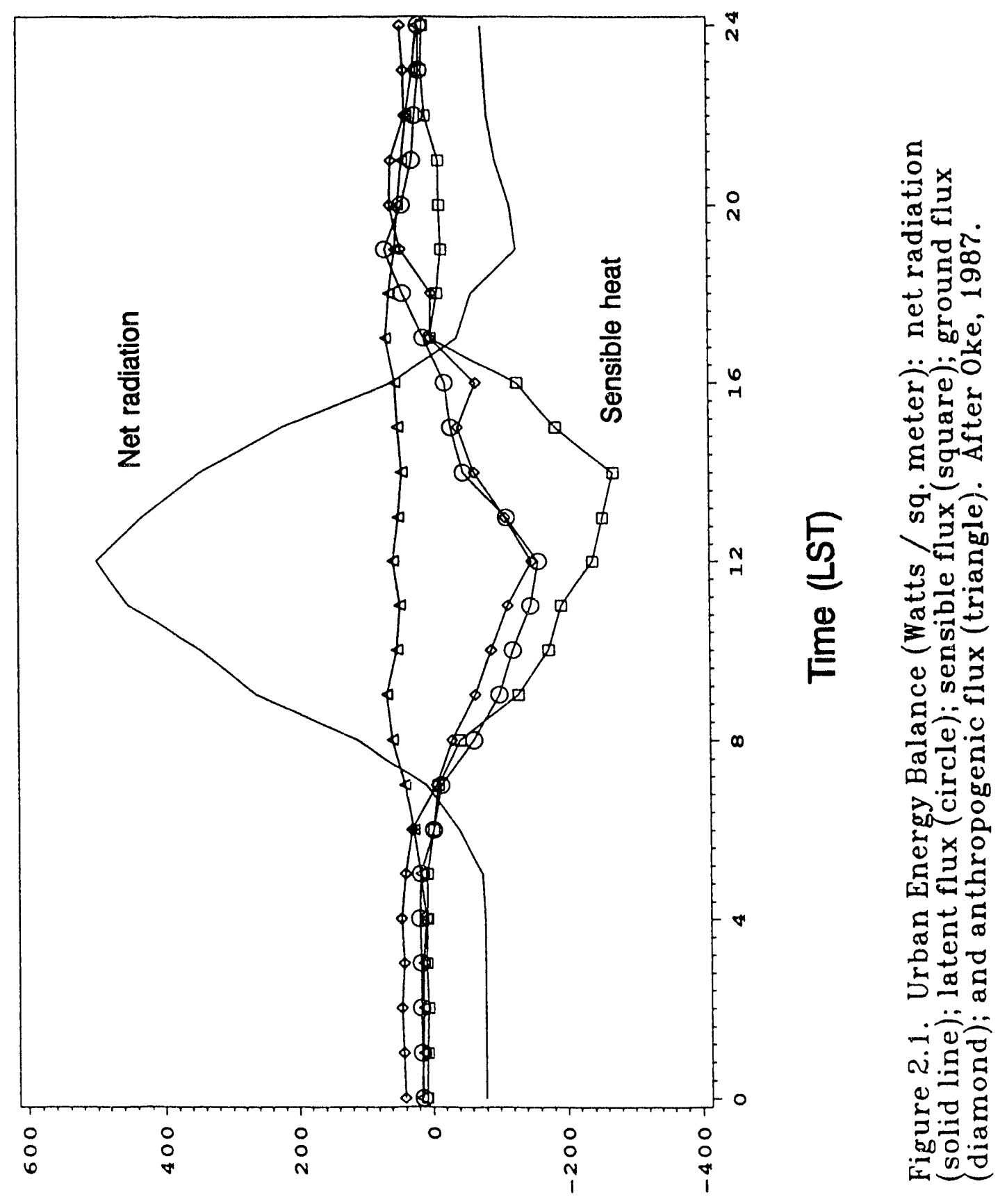

$\left(u \cdot b s / M \times n_{y}\right.$ 


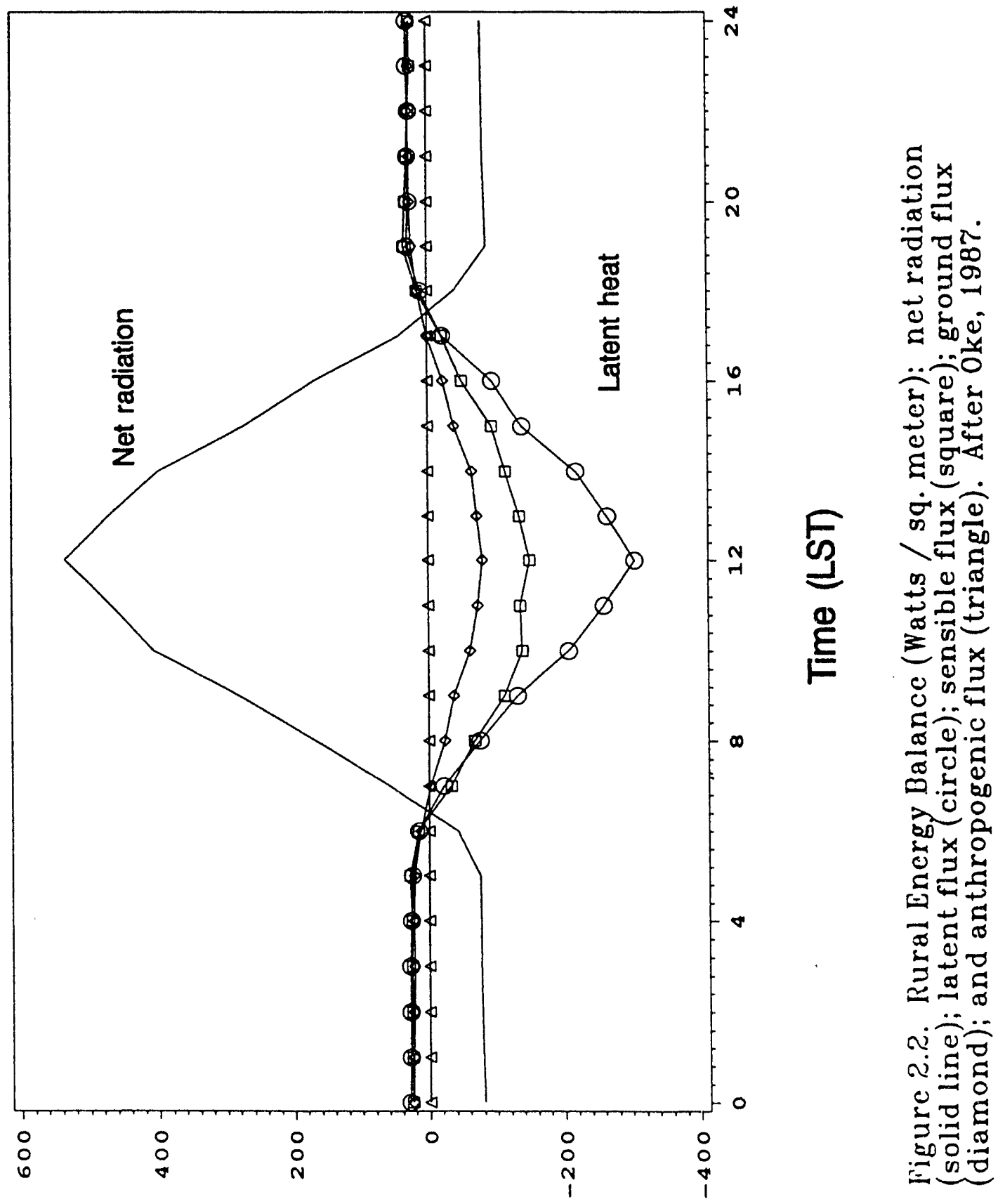

$(u \cdot b s / M \times n y$ 


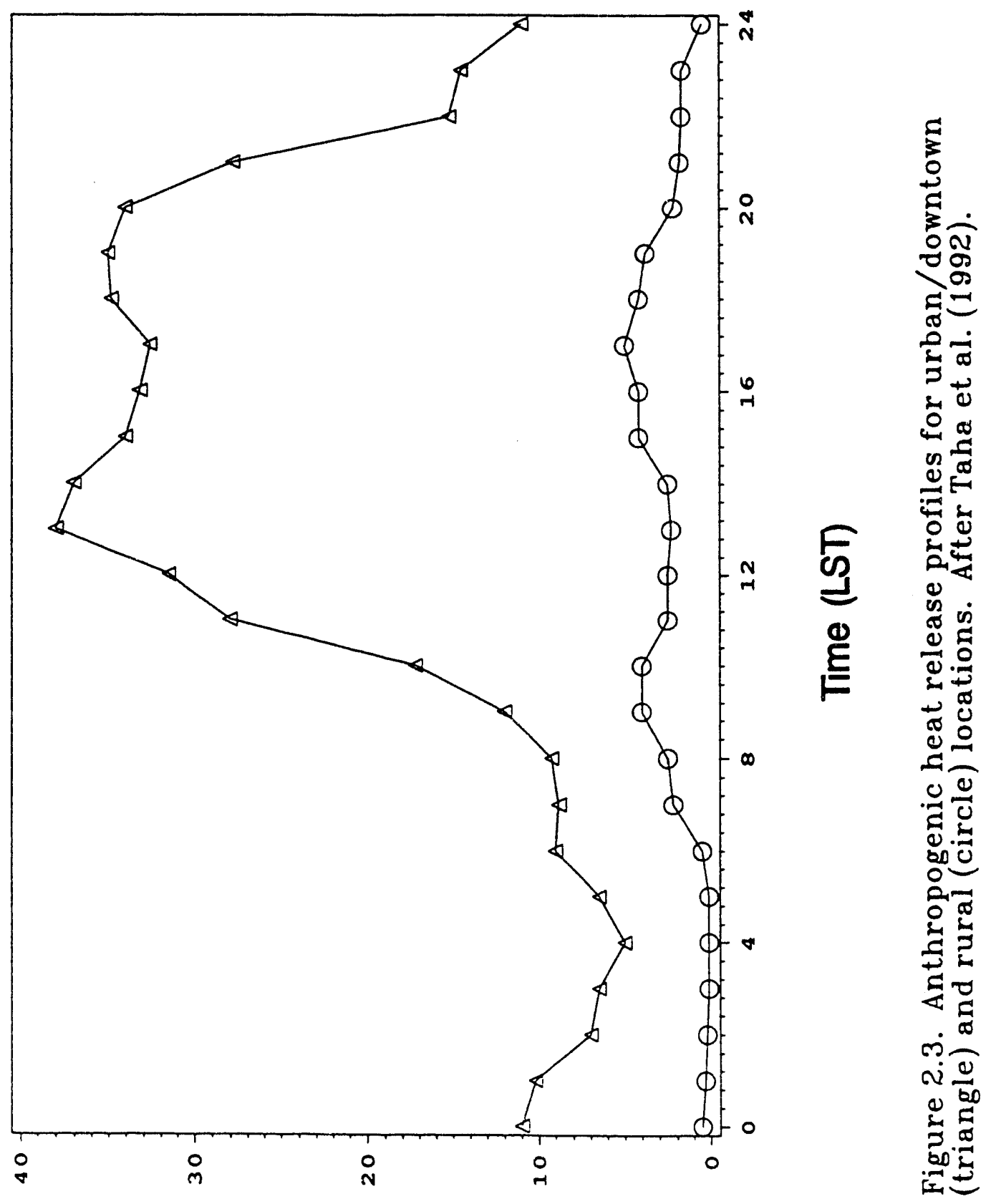

$(w \cdot b s / M$ Xn日 

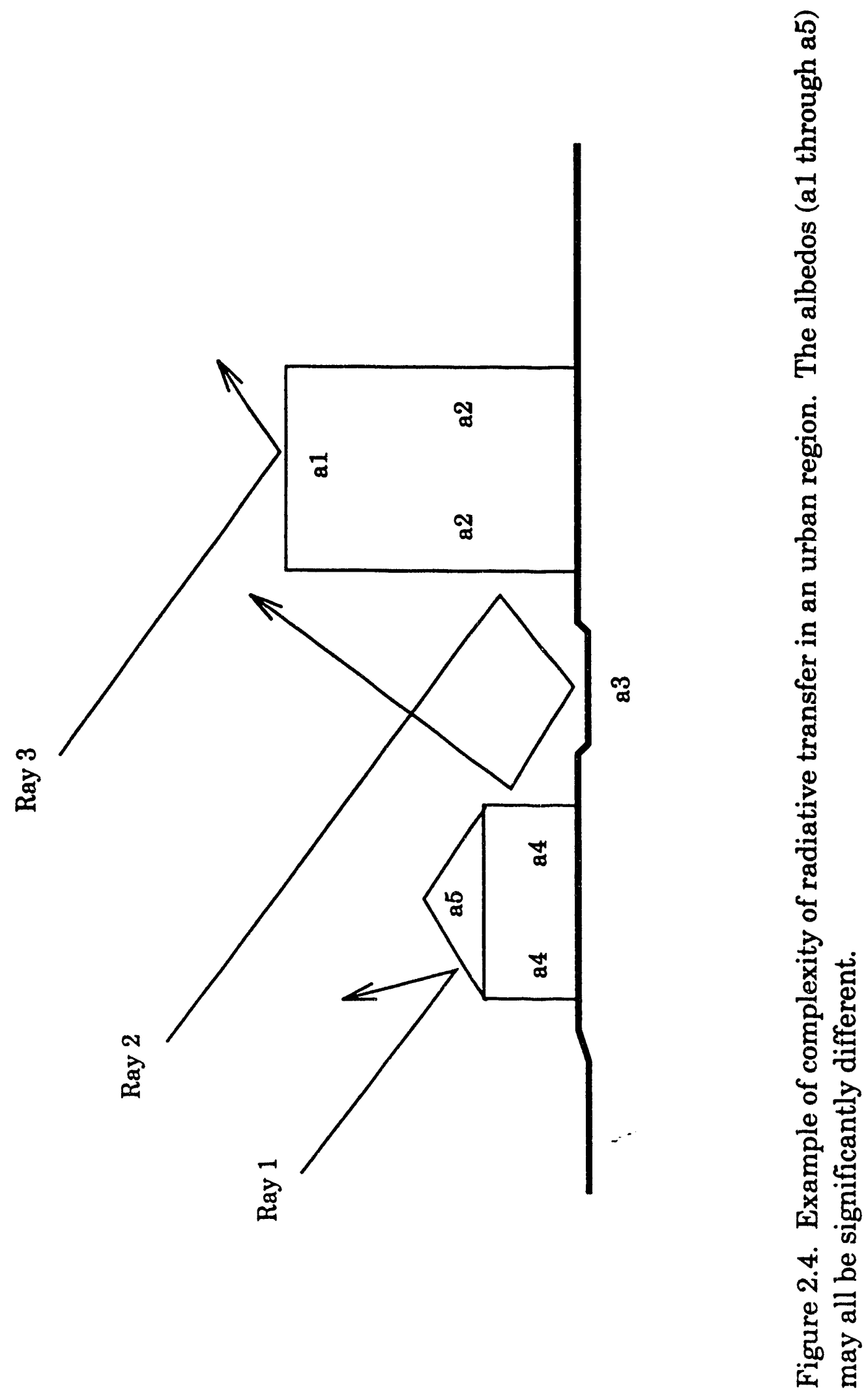


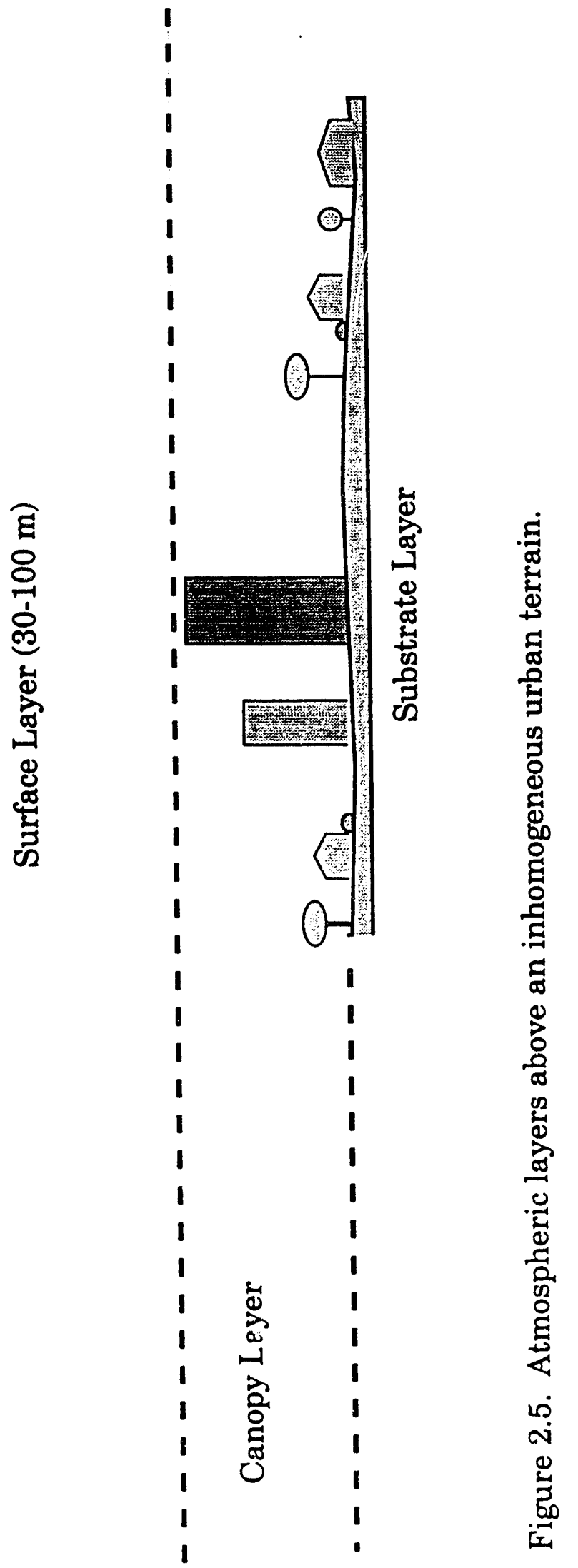




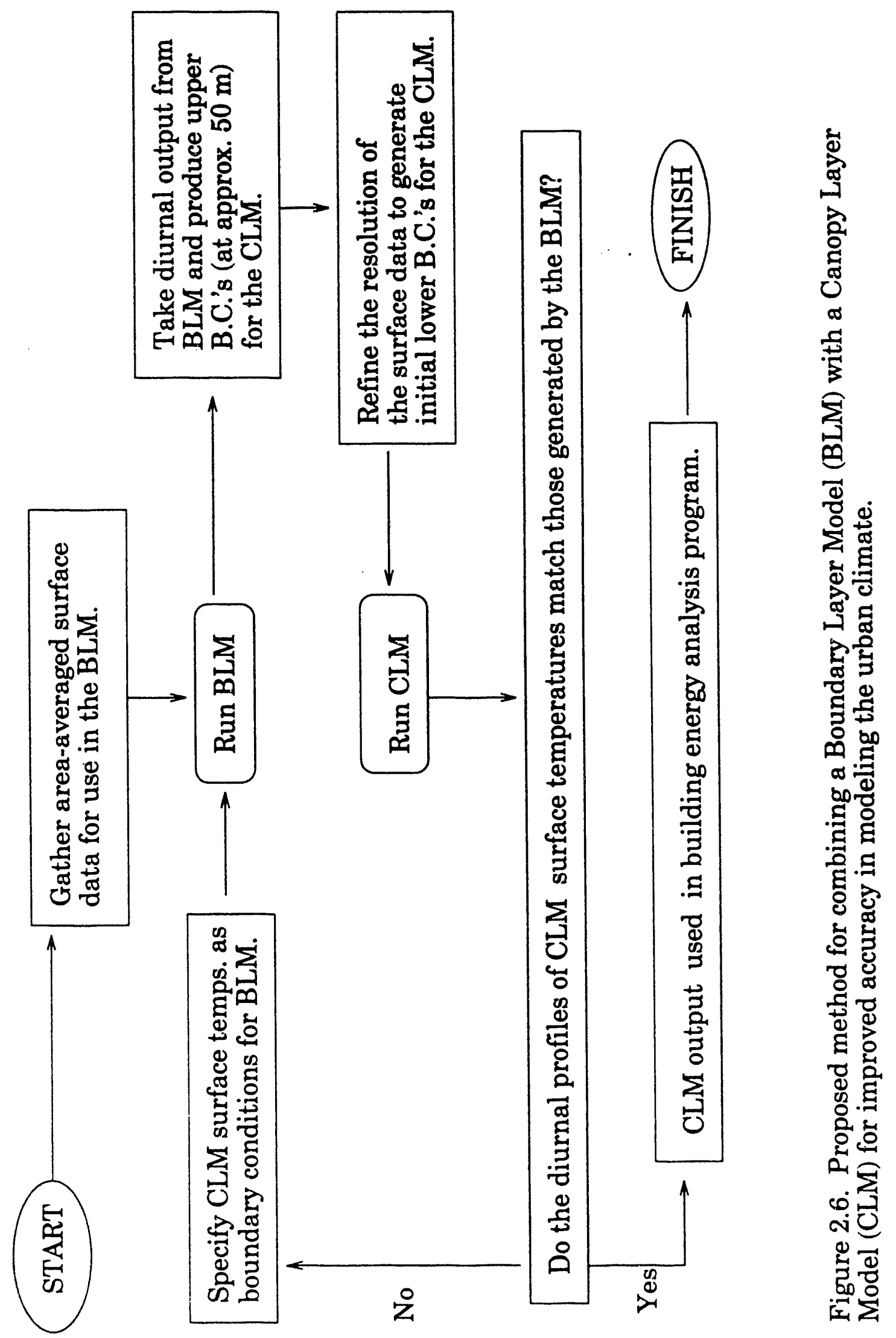




\section{CHAPTER 3 \\ Surface Characteristic Sensitivity Experiments}

\section{INTRODUCTION}

Researchers agree that albedo, surface moisture availability, substrate properties, and anthropogenic heating are among the most important factors in producing urban heat islands. In addition, cities often experience unacceptable levels of photochernical smog. This problem is exacerbated in locations characterized by large anthropogenic emissions of smog precursors and enclosed by geographical features forming a basin. Very few attempts have been made, however, to systematically evaluate the relationship between each surface characteristic and the local meteorology and air quality. This chapter and the following chapters present results from a study whose goal was to evaluate the potential of surface modifications to cool the Los Angeles Basin, save energy, and reduce photochemical smog.

Prior to conducting full three-dimensional meteorological simulations and the corresponding photochemical smog simulations, it is useful to isolate each surface characteristic and thoroughly examine its role in a developing mesometeorological coastal flow. This comprehensive series of two-dimensional simulations was used to guide the development of three-dimensional simulation cases described later. In addition, the insight gained from the analysis of this sensitivity study aids in the interpretation of meteorological results from the more complicated three-dimensional simulations. The end results of this research include: (1) an expanded understanding of the role that urbanization 
plays in affecting meteorology and air quality, and (2) the extent to which urban planners and policy makers can hope to mitigate the problems resulting from urbanization through surface modification strategies.

\section{METHODOLOGY}

A modified two-dimensional version of the Colorado State University Mesoscale Model (CSUMM) has been employed in this study. The CSUMM is a hydrostatic, incompressible, primitive-equation mesoscale meteorological model designed for simulation of airflows generated by differential surface heating and terrain irregularities. This model was originally developed by Pielke at Colorado State University (Mahrer and Pielke, 1977). Over the past two decades the CSUMM has been validated and applied in numerous situations. For a detailed description of the CSUMM see Mahrer and Pielke $(1977,1978)$ and Arritt $(1985)$. In its original form the CSUMM does not allow for grid-specific values of surface characteristics. This shortcoming was remedied by modifying the code to work with arrays of the surface characteristics and read these arrays from specified data files.

The output from the meteorological model is useful in several ways. First, it can directly indicate the impact of surface characteristics on meteorological variables such as temperature and wind speed. Secondly, the behavior of variables such as temperature, vertical wind speed, and planetary boundary-layer (PBL) height can serve as indicators of pollutant transport and diffusion. Finally, the hourly output from the meteorological model can be used as input to a photochemical model to yield quantitative predictions of the effect of surface modifications on pollutant levels. 
In this study the CSUMM was used to investigate the effects of surface modifications on the following: penetration and depth of the sea breeze; magnitude of the maximum horizontal and vertical components of wind velocity; lowlevel potential temperature; surface temperature; and effective mixing height. The potential temperature is a commonly-used meteorological variable defined as the temperature which would be attained by a parcel of dry air if it were brought adiabatically to a pressure of $100 \mathrm{kPa}$. The sea-breeze front is defined in this study as being at the location of peak positive vertical wind speed. For simulations in which an inland circulation is generated there are often several local maxima of vertical wind speed. In these cases the maxima nearest the coast is associated with the sea-breeze front.

The computational domain consisted of 64 east-west grid cells, and 22 vertical grid cells. The horizontal grid spacing was $5 \mathrm{~km}$. Vertical grid spacing was variable ranging from $25 \mathrm{~m}$ near the surface to $1000 \mathrm{~m}$ at the top of the domain which is at $9 \mathrm{~km}$ (approximately the tropopause). The time step was 60 seconds. There was a fixed boundary between an ocean covering 16 grid cells located on the western side of the domain, and land covering the remaining 48 grid cells of the domain. The computational grid is illustrated in Figure 3.1. All simulations commenced at 0300 Local Standard Time (LST) and continued until 1800 LST.

The general approach of these two-dimensional experiments was to determine the model's sensitivity to variations in several parameters at three horizontal scales of interest. These scales are domain-wide, subregional, and local. In all of the simulations the term inland is used to refer to a location well inland of the coast, and the term near ocean refers to the land surface grid points directly adjacent to the ocean. The exact locations of the various modification schemes are as follows: domain-wide (DW) modifications cover all land grid cells (cells 17-64); 
subregional near-ocean (SNO) modifications cover grid cells 17-24; subregional inland (SI) modifications cover grid cells 46-53; and localized inland (LI) modifications cover grid cells 49 and 50 .

Previous studies have used various versions of the CSUMM code to investigate urban meteorology and sea-breeze flows (Avissar and Pielke 1989; Kessler et al. 1985). In these studies, however, only very limited sensitivity studies were conducted. Kessler et al. (1985), for instance, were interested in the effects of a sandbar on sea-breeze circulations The sandbar was composed of regions of sand, clay-loam, loamy sand, dry sand, and loam . For this reason, they investigated the impact of substrate properties on the model performance.

Schultz and Warner (1982) investigated summertime circulations and pollutant ventilation in the Los Angeles Basin using a two-dimensional primitiveequation model similar to CSUMM. They present a detailed analysis of six experiments which were developed to isolate factors such as initial sounding profiles, surface characteristics, topography, and sea-breeze effects. Schultz and Warner did not attempt to disaggregate the surface characteristics in any way, however, concluding only that "correct specification of the land surface characteristics is ... important to the quality of the (meteorological) simulation(s)."

The two-dimensional simulations presented here represent the most thorough sensitivity study to date. More than twenty simulations were performed in an effort to isolate and identify the impacts of various surface characteristics on the development of a coastal meteorological flow. In particular, the sensitivity of the model to albedo, surface roughness, moisture availability, substrate properties, and anthropogenic heating was investigated. In addition to investigating domain-wide surface modifications, coastal and inland modifications ranging from 10 to $40 \mathrm{~km}$ in horizontal extent were studied. 


\section{BASE CASE}

In order to have a point of reference for the sensitivity analysis, a base case simulation was defined. Topography can significantly influence mesoscale circulations. Equally important is the impact of large-scale forcing of the geostrophic wind which can be quite variable, and in many cases can play a dominant role in the local meteorology. This geostrophic wind is the flow which results from large-scale pressure variations across the mesoscale domain. To isolate the effects of the parameters in the sensitivity tests, the large-scale geostrophic wind was set to a minimal level (constant at $10 \mathrm{~cm} \mathrm{~s}^{-1}$ ), and topography was defined as flat. The initial wind direction under such calm conditions is irrelevant and set to 0 degrees (from the North).

The initial sounding profile is given in Table 3.1. The potential temperature and specific humidity profiles correspond to a reasonable pre-dawn stable situation in the L.A. basin. The surface temperature initial condition is $292 \mathrm{~K}$.

Table 3.1: Initial pre-dawn domain-wide sounding and geostrophic wind profile

\begin{tabular}{|rcc|ccc|}
\hline $\begin{array}{c}\text { Altitude } \\
(\mathrm{m})\end{array}$ & $\begin{array}{c}\text { Potential } \\
\text { Temperature }(\mathrm{K})\end{array}$ & $\begin{array}{c}\text { Specific } \\
\text { Humidity }\end{array}$ & $\begin{array}{c}\text { Altitude } \\
(\mathrm{m})\end{array}$ & $\begin{array}{c}\text { Potential } \\
\text { Temperature }(\mathrm{K})\end{array}$ & $\begin{array}{c}\text { Specific } \\
\text { Humidity }\end{array}$ \\
\hline \hline & & & & & \\
30 & 292.0 & 0.0130 & 1200 & 313.5 & 0.0050 \\
100 & 292.1 & 0.0130 & 1500 & 315.8 & 0.0050 \\
200 & 292.2 & 0.0130 & 2000 & 316.6 & 0.0048 \\
300 & 292.6 & 0.0126 & 2600 & 316.9 & 0.0045 \\
400 & 293.8 & 0.0118 & 3200 & 318.2 & 0.0030 \\
500 & 296.0 & 0.0108 & 4000 & 320.3 & 0.0020 \\
600 & 299.3 & 0.0092 & 5000 & 323.7 & 0.0015 \\
700 & 301.3 & 0.0082 & 6000 & 327.0 & 0.0008 \\
800 & 304.8 & 0.0074 & 7000 & 330.0 & 0.0005 \\
1000 & 307.5 & 0.0060 & 8000 & 333.0 & 0.0005 \\
\end{tabular}


The substrate properties used for this base case are considered to be typical of moderately dry substrate. The thermal diffusivity, density, and specific heat capacity are $0.0028 \mathrm{~cm}^{2} \mathrm{~s}^{-1}, 1.6 \mathrm{~g} \mathrm{~cm}^{-3}$, and $0.21 \mathrm{cal} \mathrm{g}^{-1} \mathrm{~K}^{-1}$, respectively.

These simulations were intended to explore the response of the Los Angeles basin under typical summertime conditions. Therefore the Julian Day of the simulation was 221 (August 8), and the latitude was $34^{\circ}$. In addition, the default values of albedo, surface roughness, and the surface wetness parameter were $0.18,0.75 \mathrm{~m}$, and 0.10 , respectively.

\section{PARAMETRIC ANALYSIS}

The sensitivity of the model to a wide range of parametric variations was investigated. Domain-wide, subregional, and localized modifications for albedo, surface roughness, moisture availability, substrate properties, and anthropogenic heating were investigated.

\subsection{Sensitivity to Surface Characteristics}

The two-dimensional version of the CSUMM code was modified to accept input files for each of the variable surface characteristics. All of the sensitivity cases were conducted by supplying the appropriate set of input data files.

The procedures for conducting sensitivity tests of albedo, surface roughness, and moisture availability were similar. For each of these parameters six test cases were developed. The first three cases explored the impact of varying the domain-wide value of the parameter. The last three cases consisted of one large area of a single value of the parameter, and a second localized patch of a significantly different value. In this way, the domain-wide implications of localized surface characteristic modifications were investigated. 
The investigation of model sensitivity to substrate properties could have involved a complex set of experiments. The task was simplified by defining one set of substrate properties to represent an urbanized area, and another set to represent a non-urban (rural) area. For rural areas, the values of thermal diffusivity, density, and specific heat were given as $0.0026 \mathrm{~cm}^{2} \mathrm{~s}^{-1}, 1.60 \mathrm{~g} \mathrm{~cm}^{-3}$, and $0.240 \mathrm{cal} \mathrm{g}^{-1} \mathrm{~K}^{-1}$. For urban areas the corresponding values were $0.0070,1.55$, and 0.305. All of these values were based on data from Hjelmfelt (1982).

In the case of substrate properties only three test cases were defined. Two of these cases investigated subregional modifications near the ocean, and the third was designed to determine the impact of a localized inland urban region in rural surroundings.

\subsection{Sensitivity to Anthropogenic Heating}

Anthropogenic heating can be quite significant within an urban core. In particular, large cities often have summertime anthropogenic heating values on the order of $50 \mathrm{~W} \mathrm{~m}^{-2}$ (Holser and Landsberg 1977). The corresponding magnitude in rural and suburban areas is only about a tenth as large.

The magnitude of anthropogenic heating varies on a diurnal and annual basis. In the past many researchers have neglected anthropogenic heating altogether, while others, ignoring the diurnal variation, have used a single value for anthropogenic heating based on average annual energy consumption. One of the better representations of anthropogenic heating can be found in a study of the meteorology of the Tokyo bay area by Kimura and Takahashi (1991). Kimura used the diurnal variation in electric power to generate a profile for anthropogenic heat release. One drawback of the Tokyo study, however, is that it used annual values of energy use, ignoring seasonal variations. Researchers have 
shown that magnitudes often vary seasonally by $50 \%$ or more. As an example, Kalma and Newcombe (1976) estimate that the peak anthropogenic heat release for Sydney Australia on a typical winter day is about $80 \mathrm{~W} \mathrm{~m}^{-2}$, but less than 65 $\mathrm{W} \mathrm{m}^{-2}$ for a summer day.

Taha et al. (1992) developed summertime and wintertime prototypical diurnal profiles for anthropogenic heating in a hypothetical city. These profiles take into account a number of factors including energy use in the transportation sector and variations in electric power demand. In this study, a Fourier series representation $P(t)$ of their summertime profile function was used as a dimensionless scaling factor:

$$
P(t)=A_{0}+\sum_{n=1}^{3} A_{i} \operatorname{Cos}\left(\frac{2 n \pi t}{24}\right)+B_{i} \operatorname{Sin}\left(\frac{2 n \pi t}{24}\right)
$$

where,

$$
A_{0}=0.557, A_{1}=-0.227, A_{2}=-0.006, A_{3}=-0.084,
$$

and,

$$
B_{1}=-0.384, B_{2}=+0.016, B_{3}=-0.012 \text {. }
$$

In (3.1), $t$ is time in hours. This profile is shown in Figure 3.2. For each case a value of $Q$ fmax was then specified to represent the maximum value of anthropogenic heating. For a given time of day, this value is multiplied by the diurnal profile function to obtain an energy flux which is then added to the first vertical layer to augment the air temperature in this layer according to the equation:

$$
T_{1}^{+}=T_{1}+\frac{Q f \max * P}{\rho C_{p} L} * \Delta t
$$

In this equation, $\rho$ is the density of air, $C_{p}$ is the specific heat of air, $L$ is the depth of the first air layer $(25 \mathrm{~m})$, and $\Delta t$ is the time step $(60 \mathrm{~s})$. 
Domain-wide values of Qfmax from 15 to $45 \mathrm{~W} \mathrm{~m}^{-1}$ were investigated. In the localized and subregional cases, the domain-wide values of Qfmax are 0 with the exception of patches of Qfmax $=45 \mathrm{~W} \mathrm{~m}^{-1}$. In the peak, with Qfmax $=45$, (3.2) indicates that the air temperature in the lowest level will increase at a rate of $1.5^{\circ} \mathrm{C}$ per hour.

\section{RESULTS}

Although, the model can produce results at any specified time interval throughout the day, the focus of this analysis is such that conclusions can be developed from a simplified set of output data. The meteorological consequences of surface characteristics in the morning are important since the development of the sea-breeze front and the daytime growth of the boundary layer play important roles in the mixing and dispersion of precursors of photochemical smog. The afternoon meteorological impacts of surface characteristics are also of particular importance in this study since peak ozone concentrations are typically observed curing the afternoon when temperature-dependent equilibrium equation rate constants are high and incident solar radiation is still large. A study of meteorological simulations at these times also provides insight into the development of elevated summertime urban air temperatures which generate increased demand for air conditioning, resulting in elevated levels of anthropogenic heat release. In this analysis, results of the model simulations are presented for both the morning (1000 LST results) and the afternoon (1400 LST results). Due to the large anthropogenic heating in the late afternoon results from these cases at 1800 LST are also presented.

In the following discussion, reference to "low-level" air temperatures is used to indicate air temperatures which the model predicts for the first level which is 
at a nominal height of $17.5 \mathrm{~m}$.

The base case results are discussed in some detail and plots of morning and afternoon results are presented. Results of the albedo simulation cases are described in similar detail to reveal the general patterns present in the simulations. The results for the remaining cases are described in less detail.

The simulations have been separated into two categories: domain-wide cases, and localized modification cases. The base case results are summerized in detail in the section on domain-wide modifications.

\subsection{Domain-Wide Cases}

The results from the domain-wide modifications (and the base case) all have similar patterns. Since the sea breeze develops over a homogeneous land surface, each of the meteorological variables approaches an asymptotic limit far inland from the ocean interface. While air and surface temperatures and PBL heights rise from low values over the ocean to high asymptotic limits inland, horizontal and vertical velocities have low values at both ends of the computational domain, but reach peak values near the location of the sea-breeze front.

\section{BASE CASE}

The base case was developed as a reference for comparing the results of subsequent cases. This section discusses the fundamental characteristics of the morning and afternoon meteorological results for this simulation.

As the sun rises in the morning and heats the surface a sea breeze is generated due to the differential heating between the sea and land surfaces. The sea-breeze front moves steadily inland over the course of the day, bringing with it the associated mixing and advective transport of pollutants. Since the 
background geostrophic wind speed is set to a small value and a flat topographical profile is specified, all simulated large wind speeds result from differential surface heating. The location where these wind speed values start to decline is indicative of the sea-breeze front. For the purposes of this analysis the sea-breeze front is defined as being located at the point where the positive vertical wind speed is a maximum.

Figure 3.3 shows the development of the horizontal and vertical velocity profiles. The horizontal velocity profile in Figure 3.3a is a cross-section taken at an altitude of $65 \mathrm{~m}$. The vertical vclocity profile in Figure $3.3 \mathrm{~b}$ is a cross-section taken at an altitude of $350 \mathrm{~m}$. These cross-section heights were chosen somewhat arbitrarily to depict the general character of the individual velocity components. All subsequent plots of the velocity component profiles use the same crosssection heights. By 1000 LST the sea breeze has penetrated only $5 \mathrm{~km}$ inland. The peak positive wind speeds are located very near the land-water interface and are $3.98 \mathrm{~m} \mathrm{~s}^{-1}$ and $4.4 \mathrm{~cm} \mathrm{~s}^{-1}$ respectively for the horizontal (U) and vertical (W) components. The peak horizontal velocity is located at the ocean-land interface both in the morning and in the afternoon. In the afternoon, however, a second maxima, corresponding to the sea-breeze front, is evident roughly $20 \mathrm{~km}$ inland. The peak vertical velocity, however, travels with the sea-breeze front being located $35 \mathrm{~km}$ inland in the afternoon.

Both the morning and afternoon simulated planetary boundary layer heights over the ocean portion of the domain are only $30 \mathrm{~m}$. In the morning the PBL height increases to an "asymptotic" inland value of roughly $600 \mathrm{~m}$, while in the afternoon, this height has increased to $954 \mathrm{~m}$.

The development of the near-surface $(17.5 \mathrm{~m})$ air temperature for this base case is shown in Figure 3.4. The morning air temperature above the ocean 
portion of the domain is relatively constant at $18.8^{\circ} \mathrm{C}$. Starting at the land-sea interface this temperature increases asymptotically toward a value of $25.9^{\circ} \mathrm{C}$ which it reaches $25 \mathrm{~km}$ inland. The air temperature is then constant at this level throughout the remainder of the horizontal domain. The afternoon air temperature profile is similar with the same value over the ocean, but an asymptotic value which has increased by $6.3^{\circ}$ to a value of $32.2^{\circ} \mathrm{C}$ which is not reached until $90 \mathrm{~km}$ inland. The value of the air temperature over the ocean does not change from morni. $g$ to afternoon because it is strongly influenced by the ocean surface temperature which is specified as a constant boundary condition.

The surface (ground) temperature profile develops in a similar fashion. The morning ocean surface temperature has a constant boundary condition value of $18.1^{\circ} \mathrm{C}$. The first land grid point surface temperature is $26.0^{\circ} \mathrm{C}$. The surface temperature increases asymptotically toward a value of $28.8^{\circ} \mathrm{C}$ which it reaches 20 $\mathrm{km}$ inland. The surface temperature is then relatively constant at this level throughout the remainder of the horizontal domain. The afternoon surface temperature profile is similar with the same boundary condition ocean temperature of $18.1^{\circ} \mathrm{C}$. The first land grid point in the afternoon has actually cooled down slightly to $25.6^{\circ} \mathrm{C}$. This may be a result of the intensification of wind speeds in the developing sea breeze. The asymptotic value, however, has increased by $8.8^{\circ}$ to $34.4^{\circ} \mathrm{C}$. This value is not reached until roughly $65 \mathrm{~km}$ inland.

\section{DOMAIN-WIDE ALBEDO}

This section describes results for the domain-wide albedo sensitivity cases. The domain-wide albedo was increased from $10 \%$ to $30 \%$, and then to $50 \%$. This albedo increase lowered wind speeds and significantly restricted the development of the sea-breeze. Specifically, increasing albedo from $10 \%$ to $50 \%$ resulted in a decrease of roughly $30 \%$ in the horizontal wind speed peaks and of $45 \%$ in 
the vertical wind speed peaks. The corresponding effect on the sea-breeze front was a decrease in its penetration from $40 \mathrm{~km}$ inland to only $30 \mathrm{~km}$ inland. Furthermore, the growth of the PBL was significantly hampered by increasing albedo. In the afternoon, the high-albedo case resulted in a PBL height of $690 \mathrm{~m}$ which is $28 \%$ lower than the PBL height for the low-albedo case (965 m).

The low-level $(17.5 \mathrm{~m})$ air temperatures and the surface temperatures follow the typical profile, starting at a low constant value over the ocean and increasing to an asymptotic value inland. It is interesting to note that albedo modifications had only slightly more impact on the surface temperatures than on the air temperatures. Increasing albedo from $10 \%$ to $30 \%$ resulted in afternoon surface and air temperature depressions of 2.8 and $2.4^{\circ} \mathrm{C}$, respectively. Further increasing albedo from $30 \%$ to $50 \%$ resulted in additional surface and air temperature depressions of 3.3 and $2.8^{\circ} \mathrm{C}$. It is, perhaps, surprising that the surface and air temperature impacts of albedo modifications are of the same order of magnitude. Generally, one would expect the surface temperature impact to be much larger, since the albedo directly affects the energy balance of the surface, but has only a secondary impact on the air. These results imply that the modeled heat transfer between the surface and the air is quite high, resulting in only small temperature gradients. This could be a result of the relatively high surface roughness in the areas of interest. Although such an increase in domain-wide albedo would prove difficult to accomplish in practice, the results from the more reasonable range of $10 \%$ to $30 \%$ still indicate a significant impact.

\section{DOMAIN-WIDE ROUGHNESS}

The following results reveal the effect of increasing the domain-wide surface roughness from a value of $0.10 \mathrm{~m}$ to a value of $1.00 \mathrm{~m}$ and then to $1.50 \mathrm{~m}$. These simulation cases had virtually no impact on the morning circulation patterns. In 
the afternoon, however, a very slight decrease in wind speeds and PBL heights was observed to occur with increased surface roughness. This is accompanied by a slight retarding of the sea breeze penetration. If the forcing geostrophic wind were increased, the surface roughness parameter would be expected to play a more significant role.

The morning air temperature is relatively insensitive to changes in roughness whereas the afternoon air temperature decreased by $0.3^{\circ} \mathrm{C}$ when the roughness was increased from 0.10 to $1.00 \mathrm{~m}$, and by an additional $1.0^{\circ} \mathrm{C}$ when roughness was further increased to $1.50 \mathrm{~m}$. The impact of surface roughness on surface temperatures is seen to be even more significant. Increasing roughness from 0.10 to $1.00 \mathrm{~m}$ resulted in a surface temperature drop of $3.7^{\circ} \mathrm{C}$ in the morning and $4.4^{\circ} \mathrm{C}$ in the afternoon. Further increasing roughness from 1.00 to $1.50 \mathrm{~m}$ resulted in an additional temperature drop of $0.4^{\circ} \mathrm{C}$ in the morning and $0.6^{\circ} \mathrm{C}$ in the afternoon. Thus, the surface temperature was most affected by an increase in surface roughness from a very low value of $0.10 \mathrm{~m}$ to a moderate value of $1.00 \mathrm{~m}$. On the other hand, air temperature was not affected significantly by this modification range, but was quite sensitive to an increase in roughness from a moderate value of $1.00 \mathrm{~m}$ to a high value of $1.50 \mathrm{~m}$. These results suggest that in the low roughness range, sensible heat transfer from the surface to the low-level air is slightly overshadowed by vertical transport of energy out of the low-level air. In the higher roughness range, however, the additional extraction of sensible heat from the surface is significantly smaller than the additional vertical transport of energy out of the low-level air. 


\section{DOMAIN-WIDE MOISTURE AVAILABILITY}

The PBL heights are quite sensitive to domain-wide moisture availability. Increasing the moisture availability from 0.05 to 0.20 and then to 0.50 resulted in an afternoon decrease in the PBL height from 1100 to 772 to $682 \mathrm{~m}$. The corresponding decreases in peak horizontal wind speeds were $18 \%$ and $31 \%$. The vertical wind speed was even more sensitive to changes in moisture availability, decreasing from $7.8 \mathrm{~cm} \mathrm{~s}^{-1}$ by $35 \%$ and $57 \%$ for this series of modifications.

The surface temperature was found to be very sensitive to moisture availability. Increasing moisture availability from 0.05 to 0.20 resulted in a surface temperature depression of $6.2^{\circ} \mathrm{C}$ at 1400 LST. Further increasing moisture availability from 0.20 to 0.50 depressed the surface temperature by an additional 3.1 ${ }^{\circ} \mathrm{C}$. We found that air temperatures were nearly as sensitive to moisture availability. Specifically, increasing domain-wide moisture availability from 0.05 to 0.20 , and then to 0.50 lowered air temperatures by $5.0^{\circ} \mathrm{C}$ and $7.7^{\circ} \mathrm{C}$, respectively.

\section{DOMAIN-WIDE ANTHROPOGENIC HEATING}

For the following simulations the domain-wide anthropogenic heating profiles followed the form illustrated in Figure 3.2. The peak of this profile was designated as 15,30 , and $45 \mathrm{~W} \mathrm{~m}^{-2}$ for the three domain-wide anthropogenic heating simulations. The development of the sea breeze was surprisingly insensitive to the level of imposed anthropogenic heating. In fact, compared with the base case, the most significant impact on the sea breeze occurred in the morning when the specification of a $45 \mathrm{~W} \mathrm{~m}^{-2}$ peak heating profile resulted in an increase of roughly $10 \%$ in both the PBL height and the peak vertical velocity. The corresponding impact on peak horizontal velocities was virtually undetectable, a decrease of roughly $2 \%$. This could be simply within the range of computational 
error. In contrast to this result, in the afternoon and in the evening, the PBL height and wind speeds for these cases were nearly identical to those of the base case. These results are unexpected since the anthropogenic heating term follows a diurnal profile which peaks late in the day (between 1300 and 1700 LST) and is only at about $40 \%$ of its peak at 1000 LST.

Each $15 \mathrm{~W} \mathrm{~m}^{-2}$ increase in the nominal value of the anthropogenic heating profile resulted in a $0.2^{\circ} \mathrm{C}$ increase in air and surface temperatures both in the morning and in the afternoon. In the evening (6:00 p.m.), however, the air and

surface temperatures increased by 0.5 and $0.3^{\circ} \mathrm{C}$ respectively for each $15 \mathrm{~W} \mathrm{~m}^{-2}$ increase in anthropogenic heating.

Thus anthropogenic heating has minimal impact on the development of the daytime sea breeze and only a moderate impact on evening air temperatures. The slight increase in morning PBL height has positive air quality implications while the small increases in air temperatures may counteract this effect. These results suggest that anthropogenic heating does not play a dominant role in the daytime formation of photochemical smog. The increase in evening air temperatures may, however, be responsible for a significant increase in the demand for air conditioning electricity.

\subsection{Localized Modifications}

The localized modification cases produced distinctly different results relative to the domain-wide cases. In the near-ocean cases, the region over which the meteorological variables approached their asymptotic limit was extended. In the inland cases, pronounced temperature and PBL height depressions were often produced over the location of the modification. The horizontal extent of these depressions generally corresponded to the length of the modification zone. In 
addition, these cases also produced significant impacts on the magnitudes of the local winds.

\section{LOCALIZED ALBEDO}

The localized and subregional albedo cases investigated the impact of small scale (either inland or near the ocean) albedo modifications. In the subregional near-ocean (SNO) albedo modification case the land surface grid points nearest the ocean had a high albedo of $50 \%$ and the rest of the land surface had a low albedo of $10 \%$. Figure 3.5 demonstrates the development of the horizontal and vertical velocity profiles for the SNO case. While the wind speeds in this case are generally lower than those from the base case, the penetration of the sea-breeze front appears to be significantly enhanced as a result of the near-ocean albedo modification. In the afternoon the sea-breeze front is located $70 \mathrm{~km}$ inland for the SNO case, but only $40 \mathrm{~km}$ inland for the base case.

The impacts of subregional inland (SI) and localized inland (LI) regions of high albedo were also investigated. In both cases, the albedo modification succeeded in producing its own circulation pattern. These circulation patterns are clearly visible in velocity component profile plots. Figure 3.6 shows the afternoon horizontal and vertical velocity components across the simulation domain for the SI case. While not as large as the sea breeze, the circulation generated by the surface modification is quite distinct and extensive. The resulting circulation resembles a fluid jet impinging downwards upon the modification zone, with flow vertically toward and horizontally away from this zone.

Figure 3.7 contains the corresponding plots for the modification of the LI case. Even though this modification is only $10 \mathrm{~km}$ in extent, its impact on both velocity fields extends roughly $25 \mathrm{~km}$ in both directions. The generated 
circulation of the LI case has a horizontal velocity profile which is similar to that of the SI case, but with about half the magnitude. The vertical velocity profile, however, shows a very interesting result. The generated circulation of the LI case has a peak downward velocity of $5.1 \mathrm{~cm} \mathrm{~s}^{-1}$ compared with only 3.7 for the SI case, indicating that the localized albedo modification had more of an impact on vertical flow than the subregional modification. Thus, regions of highly variable albedo may hold the potential for increased vertical mixing and improved air quality due to the interactions between circulations generated by neighboring, differentially heated surfaces.

In addition to the impact on wind speeds, all three modifications resulted in substantial depressions in the PBL height. The near-ocean albedo modification produced a pronounced morning impact on the PBL height. This $50 \mathrm{~km}$ long modification resulted in a $50 \mathrm{~km}$ long depression of the PBL height from the asymptotic value of $670 \mathrm{~m}$ to a reduced value of only $490 \mathrm{~m}$. It took roughly 15 $\mathrm{km}$ on either side of the modification for the PBL height to change from the asymptotic value to the depression value. In the afternoon this depression was smoothed out somewhat, but was still very pronounced. When the same albedo modification was implemented inland (SI case) the resulting impact on the PBL height was similar. In the morning the PBL dipped $180 \mathrm{~m}$ and in the evening it was reduced by $385 \mathrm{~m}$. The LI case, having a very localized modification, resulted in a very localized impact on the PBL. In the morning the result was a dip of $110 \mathrm{~m}$ in the PBL height, whereas in the evening this depression had a magnitude of $230 \mathrm{~m}$.

Since the modification of the SNO case was located in the region where the boundary layer grows rapidly it is difficult to determine the extent of the PBL depression in this case. This problem occurs in many of the profiles for each of 
the near-ocean modification cases.

The air temperature impact of the localized albedo modifications is demonstrated in Figure 3.8. The total extent over which the surface modification had a measurable impact on air and surface temperatures is larger than the corresponding area of PBL impact. In the afternoon, for example, the inland modification of the SI case had an impact on the air temperature $25 \mathrm{~km}$ upstream and $30 \mathrm{~km}$ downstream of the modification zone.

Since the albedo directly affects the surface energy balance, and only has a secondary impact on air temperatures, it was expected that the impact of these inland albedo modifications on the surface temperatures would be stronger than the impact on air temperatures. The fact that these results are of the same magnitude is surprising. This inconsistency may point to an inadequacy in the model, or may be indicative of the complex physical interactions between the heterogeneous surface and the local meteorology.

\section{LOCALIZED ROUGHNESS}

In the subregional near-ocean (SNO) roughness case the land surface grid points nearest the ocean were given a relatively high roughness length of $1.50 \mathrm{~m}$ while the remainder of the land surface had a low roughness value of $0.10 \mathrm{~m}$. The morning and afternoon wind vector profiles for this case are not substantially different from those of the base case.

The impacts of subregional and localized inland regions of high roughness were also investigated. The results indicate that the inland roughness modifications had very little impact on wind speeds in the morning and produced moderate increases (less than 10\%) in the level of local winds in the afternoon. 
The impact of the inland modifications on air temperatures was negligible in the morning and of only moderate importance in the afternoon. The impact on surface temperatures, however, was quite large both in the morning and the afternoon. Specifically, the SI case resulted in morning and afternoon surface temperature depressions of 4.3 and $4.9^{\circ} \mathrm{C}$ respectively. The LI case produced surface temperature depressions which were almost as large: $4.2^{\circ} \mathrm{C}$ in the morning, and $4.5^{\circ} \mathrm{C}$ in the afternoon.

\section{LOCALIZED MOISTURE AVAILABILITY}

The localized moisture availability modifications resulted in strong surfacegenerated circulation patterns. The subregional inland case tested a $40 \mathrm{~km}$ long region of 0.50 moisture availability in an otherwise dry (0.05) land domain. The localized inland case was similar except that the high moisture region spanned only $10 \mathrm{~km}$. The large extent of the modification in the SI case was responsible for larger horizontal velocities than those induced by the LI case. On the other hand, the LI case generated a stronger vertical circulation. Similar to the results of the albedo cases, these simulations imply that areas of limited extent, but highly variable moisture content can produce significant vertical circulations which will result in enhanced vertical mixing.

The subregional modification resulted in larger air and surface temperature depressions than the local modification. In the SI case the morning air and surface temperature depressions were 4.0 and $5.9^{\circ} \mathrm{C}$, while the corresponding depressions for the LI case were only 2.6 and $4.8^{\circ} \mathrm{C}$. This trend continued into the afternoon when the SI case air and surface temperature depressions were 7.0 and $8.7^{\circ} \mathrm{C}$ relative to 4.0 and $6.4^{\circ} \mathrm{C}$ for the LI case. So, the impact of the moisture modifications on surface temperatures was only slightly larger than the impact on air temperatures. 


\section{LOCALIZED SUBSTRATE PROPERTIES}

As discussed previously two types of substrate were defined, urban and rural. One SNO case specified that the near-ocean grid cells were composed of urban substrate while the remaining cells were composed of rural substrate. Another SNO case reversed this scenario. The LI case specified a localized region inland which was composed of urban substrate while the remaining cells contained rural substrate.

The peak wind speeds and the development of the sea breeze are very similar for the two SNO cases in the morning. In the afternoon there is a noticeable, but small difference between these two cases.

In the first $\mathrm{SNO}$ case the PBL begins its growth over urban substrate. In the other SNO case the PBL begins its growth over rural substrate. The growth of the PBL in the morning for these two cases is nearly identical with the second case growing at a slightly faster rate and reaching an asymptotic peak of a slightly lower value. In the afternoon the PBL for the second case initially grows at a slower rate and reaches a lower asymptotic PBL height than that for the first case.

The LI case investigated the impact of a localized inland region of urban substrate properties surrounded by rural substrate properties. The impact of this region on wind speeds was only slight. A small region of very slow downward flow was produced over the urban region. In addition, this region produced a very slight dip in the PBL from an asymptotic value of $600 \mathrm{~m}$ to a value of $593 \mathrm{~m}$.

The near-ocean substrate modifications had a surprising impact on the asymptotic values of both surface and air temperatures. In the afternoon, the first SNO case (urban near the ocean) resulted in asymptotic surface and air temperatures which were roughly $0.6^{\circ} \mathrm{C}$ higher than for the second SNO case (rural 
near the ocean). Although the temperature differences between these two cases are not large they are significant considering that they occur in a region wellremoved from the modifications and in the presence of a wind field that is only slightly affected by the modifications.

The urban substrate region in the LI case is responsible for a slight dip in both the surface and air temperature profiles. The air temperature decreases by roughly $0.2^{\circ} \mathrm{C}$ in this region both in the morning and the afternoon. The surface temperature decreases by $0.4^{\circ} \mathrm{C}$ in this region in the morning and by $0.5^{\circ} \mathrm{C}$ in the afternoon.

\section{LOCALIZED ANTHROPOGENIC HEATING}

In contrast to the localized surface characteristic cases, the localized and subregional anthropogenic heating cases resulted in elevations of PBL height and temperatures rather than depressions. As was the case in the domain-wide anthropogenic heating cases, the SI and LI anthropogenic heating cases had negligible impact on the afternoon meteorological variables. In the morning, however, the PBL height increased over the modification zones. For the SI case, the PBL height grew to $673 \mathrm{~m}$ relative to $606 \mathrm{~m}$ for the base case. The corresponding PBL height for the LI case was $645 \mathrm{~m}$.

Similar to the domain-wide cases, the air and surface temperatures were only slightly affected by the subregional and local anthropogenic heating modifications. The resulting temperature elevations were on the order of 0.1 or $0.2^{\circ} \mathrm{C}$ both in the morning and the afternoon. In the evening (1800 LST) these temperature elevations were only slightly larger, in the range of 0.3 to $0.5^{\circ} \mathrm{C}$. 


\section{SUMMARY OF SENSITIVITY RESULTS}

The results of these two-dimensional simulations have indicated the scope and relative importance of the effects of various surface characteristics on local meteorology. These results can be aggregated into five distinct categories: albedo; surface roughness; substrate moisture; substrate properties; and anthropogenic heating. Domain-wide modifications of these properties resulted in domain-wide variations of temperature, PBL heights, and wind speeds. The localized modifications generally had impacts of similar magnitude relative to the domain-wide modifications, but their horizontal extents were limited by the extent of the surface modification.

The simulations show that surface albedo has a significant impact on both afternoon temperatures and local winds. Increasing domain-wide albedo from $10 \%$ to $50 \%$ resulted in red 1 as of $30 \%, 50 \%$, and $28 \%$ in horizontal peak wind speeds, vertical peak wind speeds, and PBL heights respectively. The corresponding impact on surface and air temperatures was also large, reaching 6.1 and $5.2^{\circ} \mathrm{C}$ respectively in the afternoon.

The sensitivity to roughness variations was less substantial. Increasing domain-wide surface roughness from 0.10 to $1.50 \mathrm{~m}$ had virtually no impact on peak wind speeds or PBL heights. In the morning the impact of roughness on air temperatures was insignificant, but the impact on surface temperature reached $4.2^{\circ} \mathrm{C}$. In the afternoon, however, these two roughness cases resulted in a reduction of surface and air temperatures by up to 5.0 and $1.3^{\circ} \mathrm{C}$ respectively. Thus, we conclude that the impact of surface roughness is essentially limited to surface temperatures.

The moisture availability sensitivity cases demonstrated the most significant impact on meteorology. Increasing domain-wide moisture availability from 0.05 
to 0.50 resulted in reductions of $31 \%, 57 \%$, and $38 \%$ in horizontal peak wind speeds, vertical peak wind speeds, and PBL heights, respectively. The corresponding impact on surface and air temperatures was also large. For the likely range of moisture availability values $(0.05$ to 0.20$)$ the air and surface temperature sensitivities were 3.3 and $4.1^{\circ} \mathrm{C}$ per 0.1 change in surface moisture.

The impact of substrate properties on the local meteorology appears to be insignificant. The urban substrate case resulted in peak horizontal wind speeds, peak vertical wind speeds and PBL heights which were only slightly different from the rural substrate case. Interestingly, the wind speeds were slightly higher for the rural substrate case, but the PBL heights were slightly higher for the urban substrate case. This result is unique since for each of the other sensitivity parameters, high wind speeds were paired with high PBL heights. The urban substrate case also resulted in surface and air temperatures which were no more than $0.7^{\circ} \mathrm{C}$ higher than the rural substrate case. It should be pointed out, however, that these minimal simulated meteorological impacts resulting from substrate property differences may be on the same order as the computational accuracy of the model, and may simply represent erroneous fluctuations associated with computational convergence.

The addition of moderate levels of anthropogenic heating had relatively little impact on the developing sea breeze. Anthropogenic heating also had virtually no impact on surface or air temperatures in the morning and afternoon. In the evening, however, the addition of a reasonable level of anthropogenic heating increased the low-level air temperature by $1.5^{\circ} \mathrm{C}$.

The inland modifications demonstrated the ability to generate their own circulations independent of the sea breeze. In particular, the inland albedo modification cases resulted in a relatively strong flow near the region of albedo 
contrast. In addition, a large depression in PBL height was observed over the high-albedo regions. In general, such a PBL depres ;ion might be expected to have a negative impact on air quality since it would be representative of lower levels of atmospheric mixing. These simulations also resulted in air and surface temperature depressions, hewever, which should have a positive impact on air quality by lowering the photochemical rate constants, thus slowing the production of photochemical smog. These opposing results were also observed to be significant in the moisture availability cases.

Thus the surface characteristics which were investigated in this study have been shown to affect local meteorology in a complex manner. In order to determine the net effect of these surface characteristics on air quality photochemical simulations are necessary. In addition, full three-dimensional meteorological simulations are needed to explore the significance of topographical variations. 


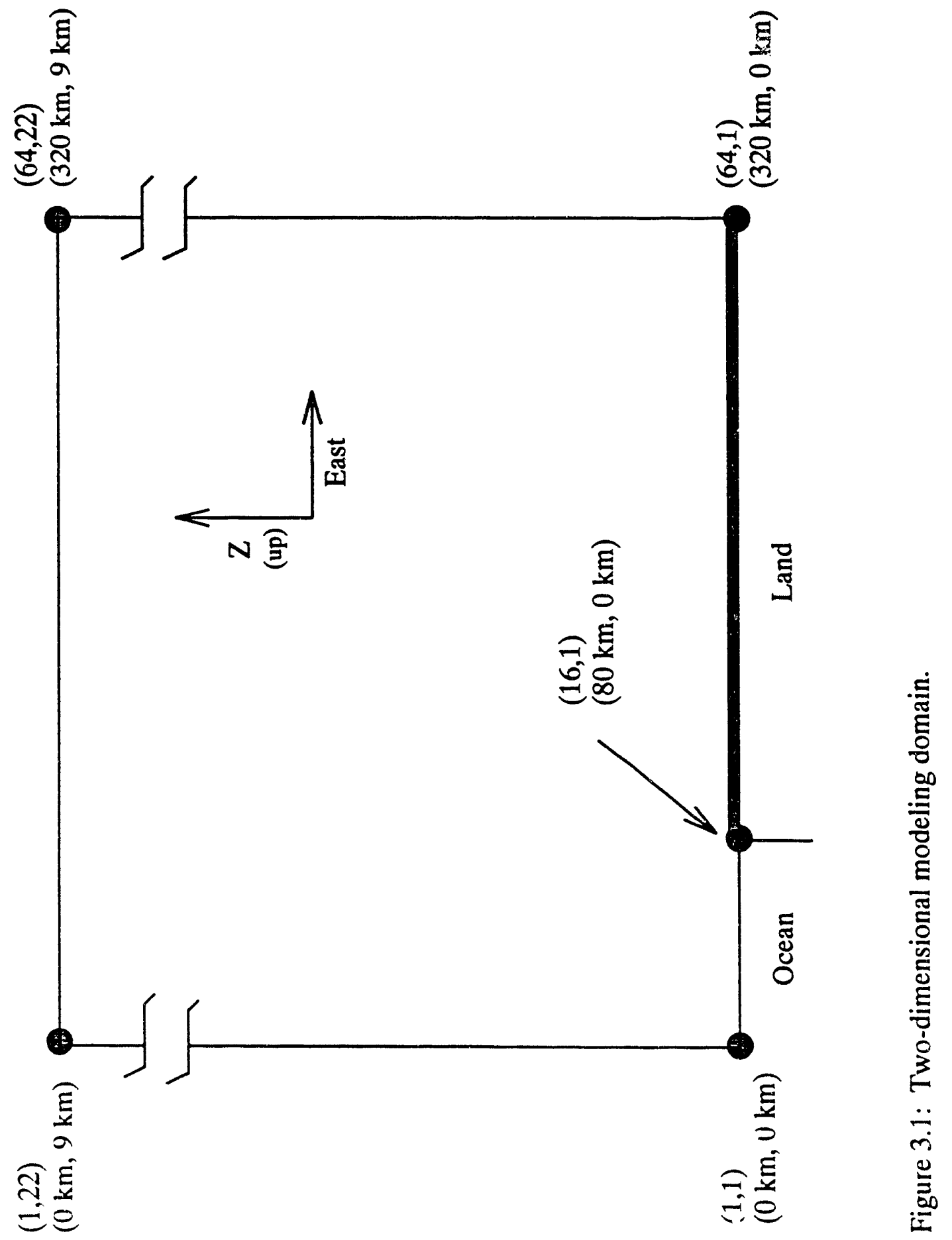



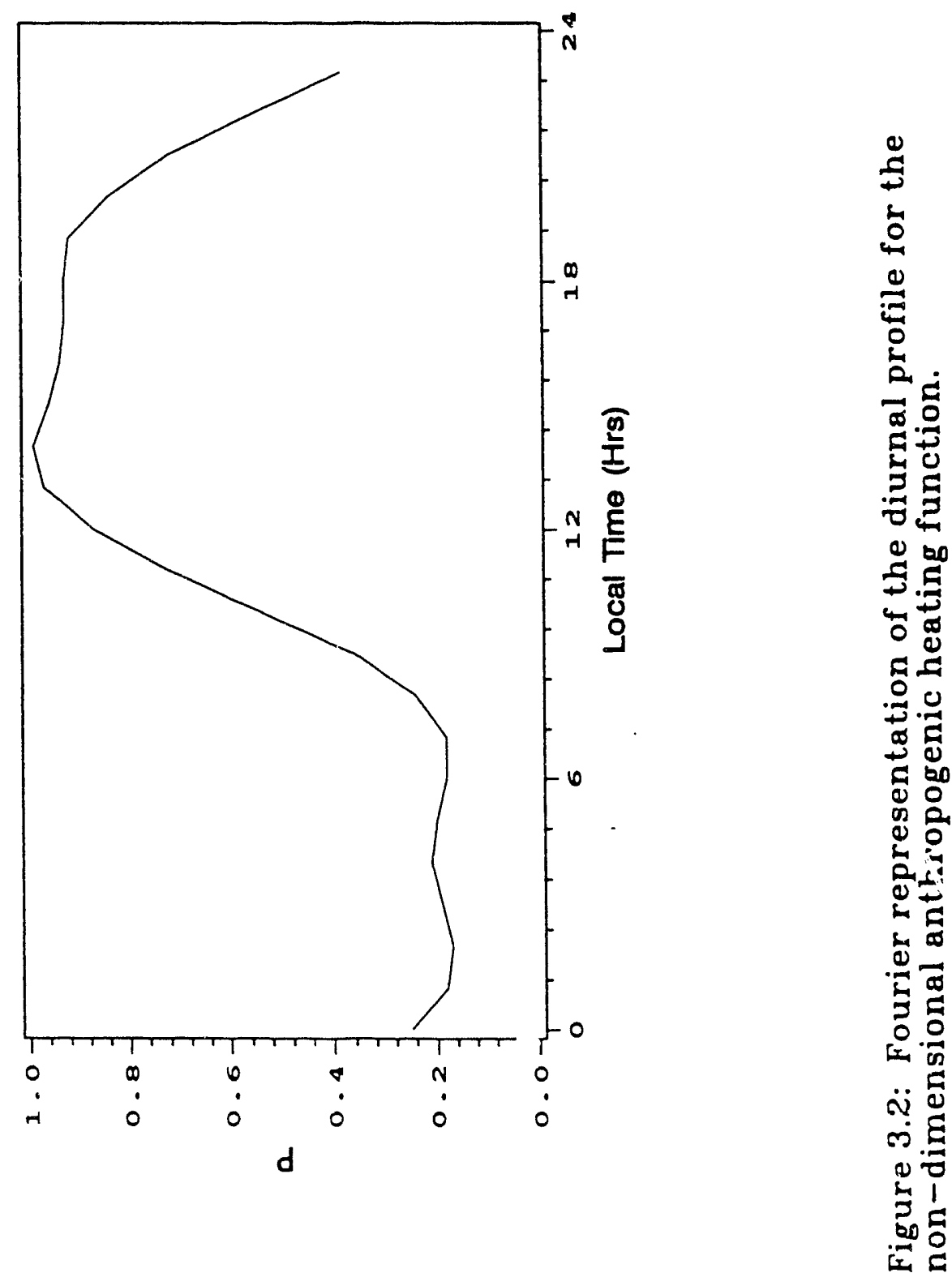


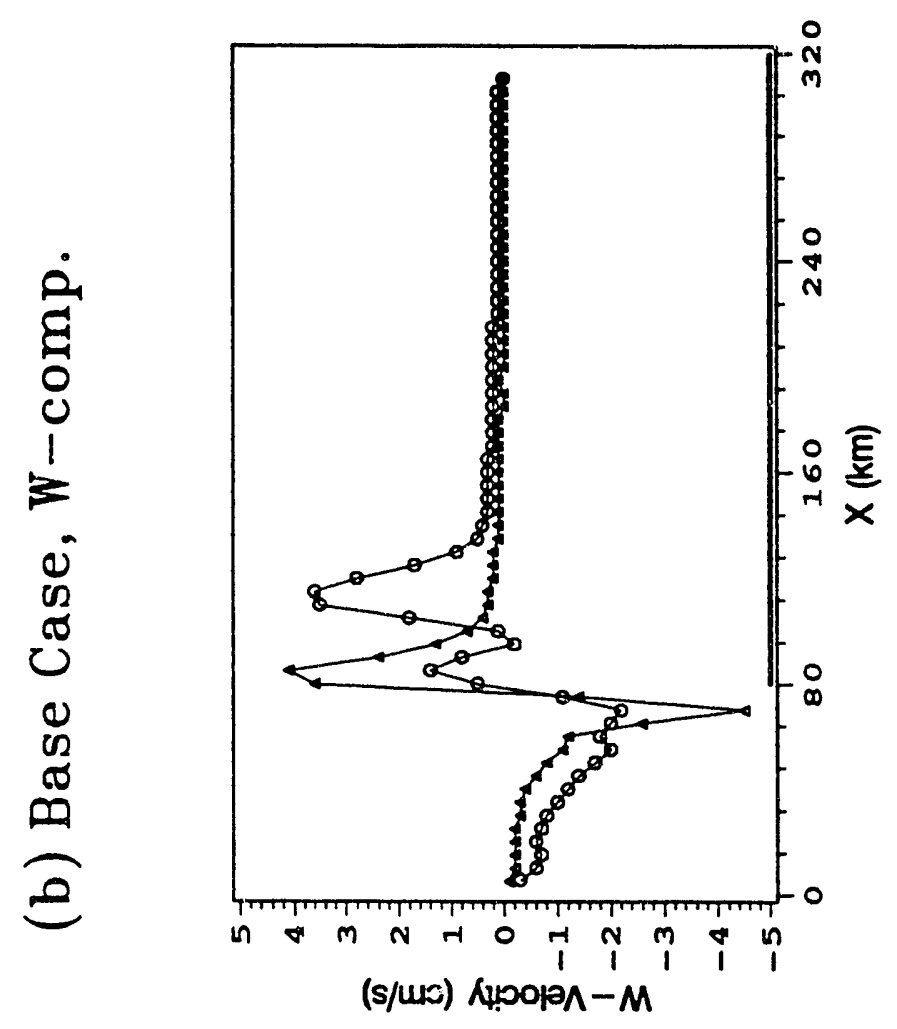

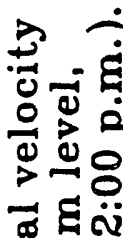
등 एँ Dे둥 0 명ำ ๘ $>$ Фేฐ요 击 소음 in

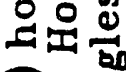
ฮ.

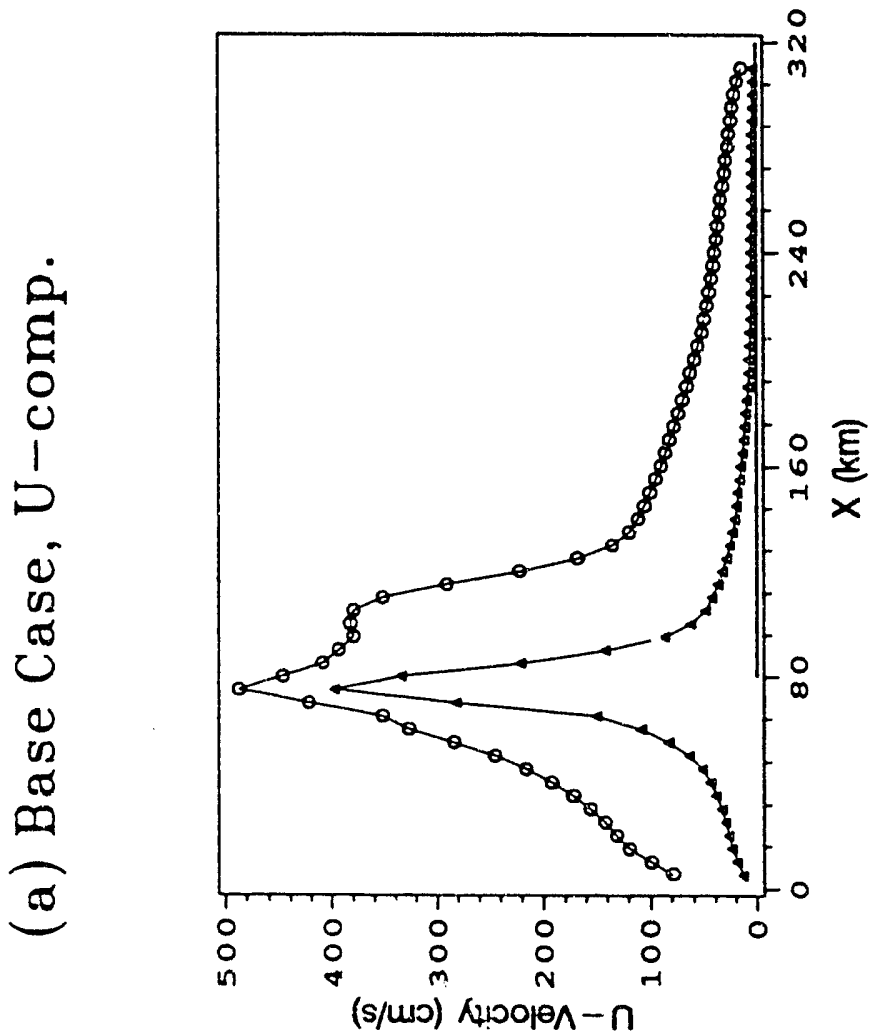
동호 $\circ \Phi$ घี่ 政 品 表号 ด0 है 四 击 के 㐘 ติ क 잉 可 일 

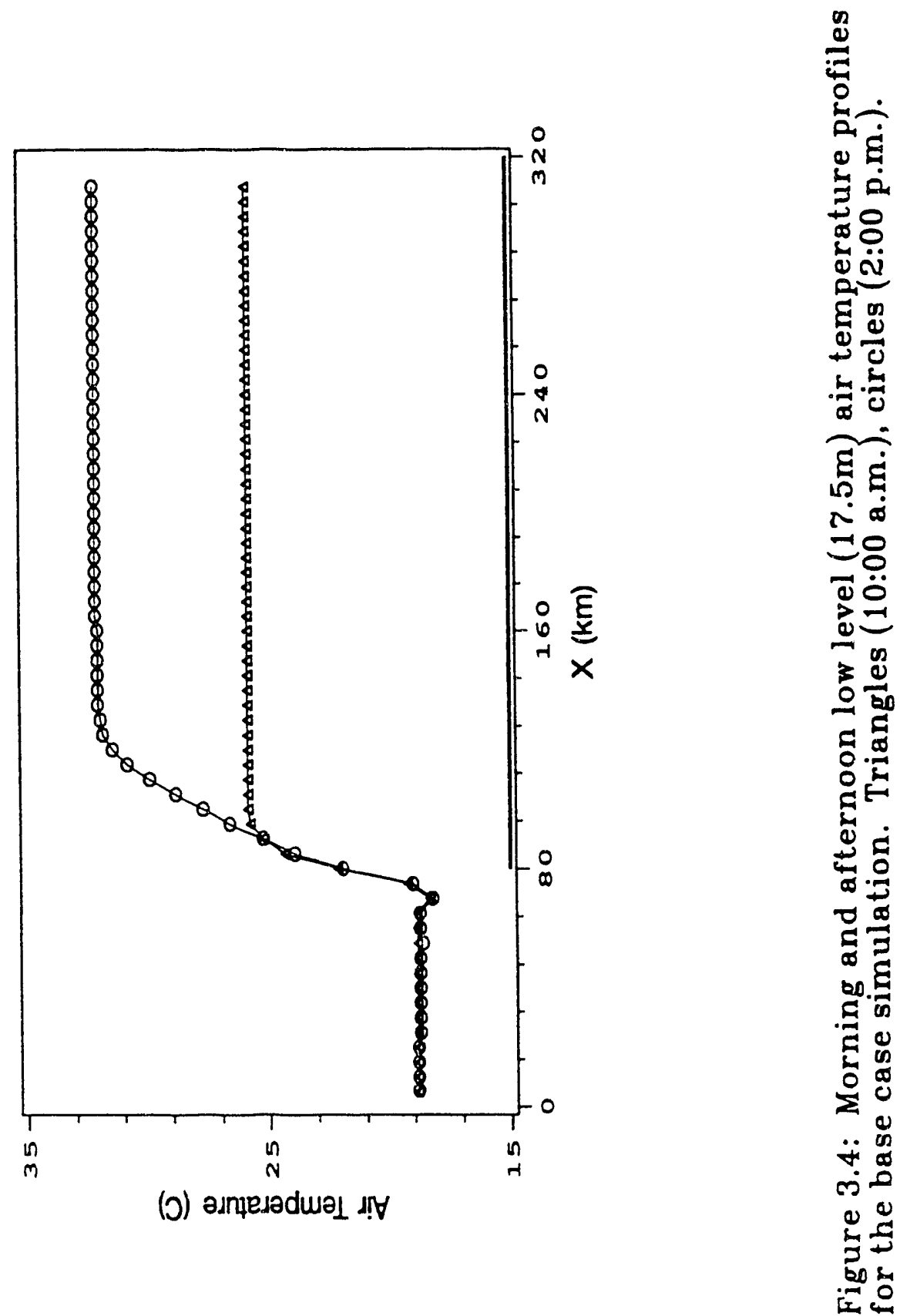

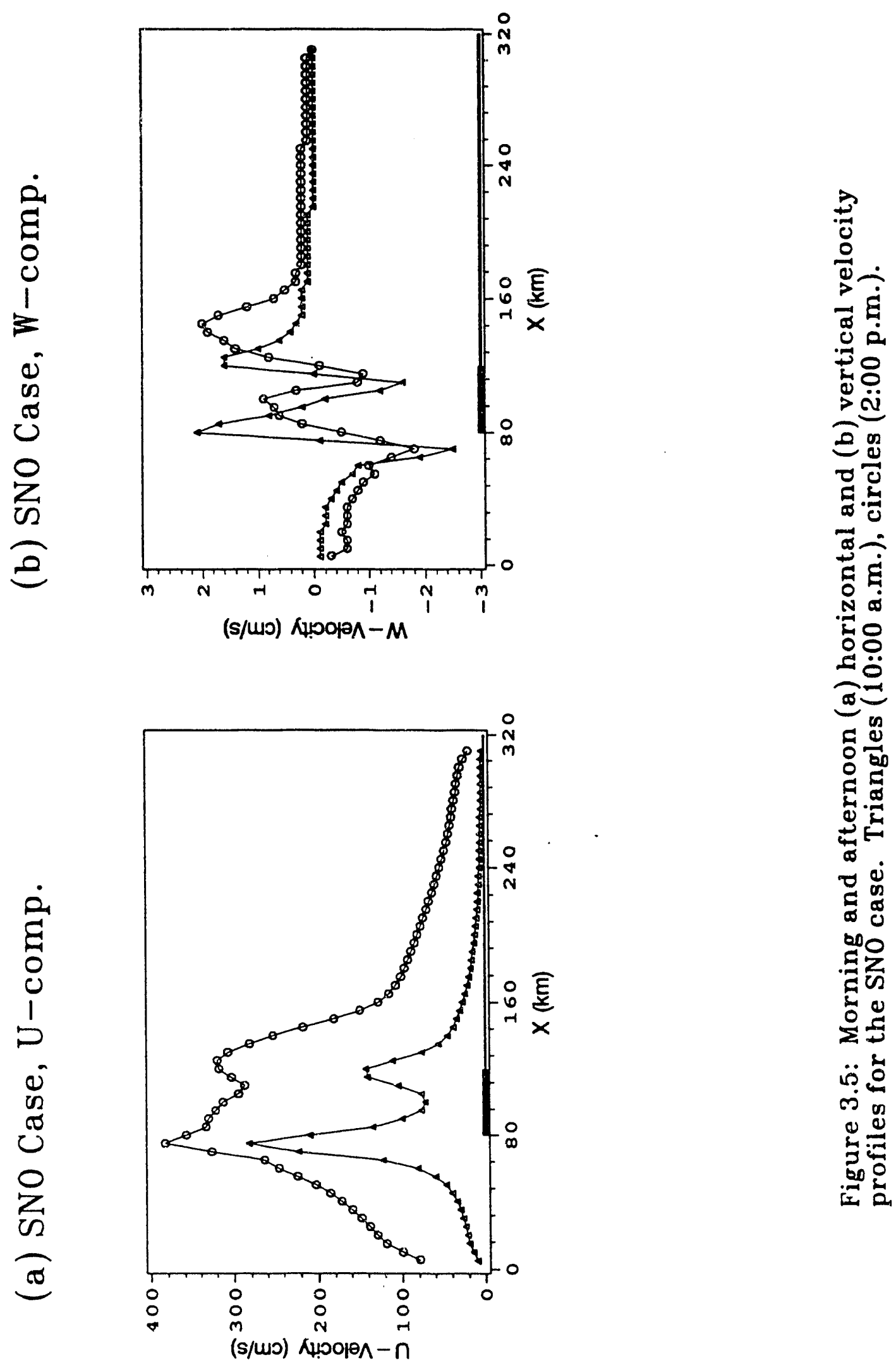


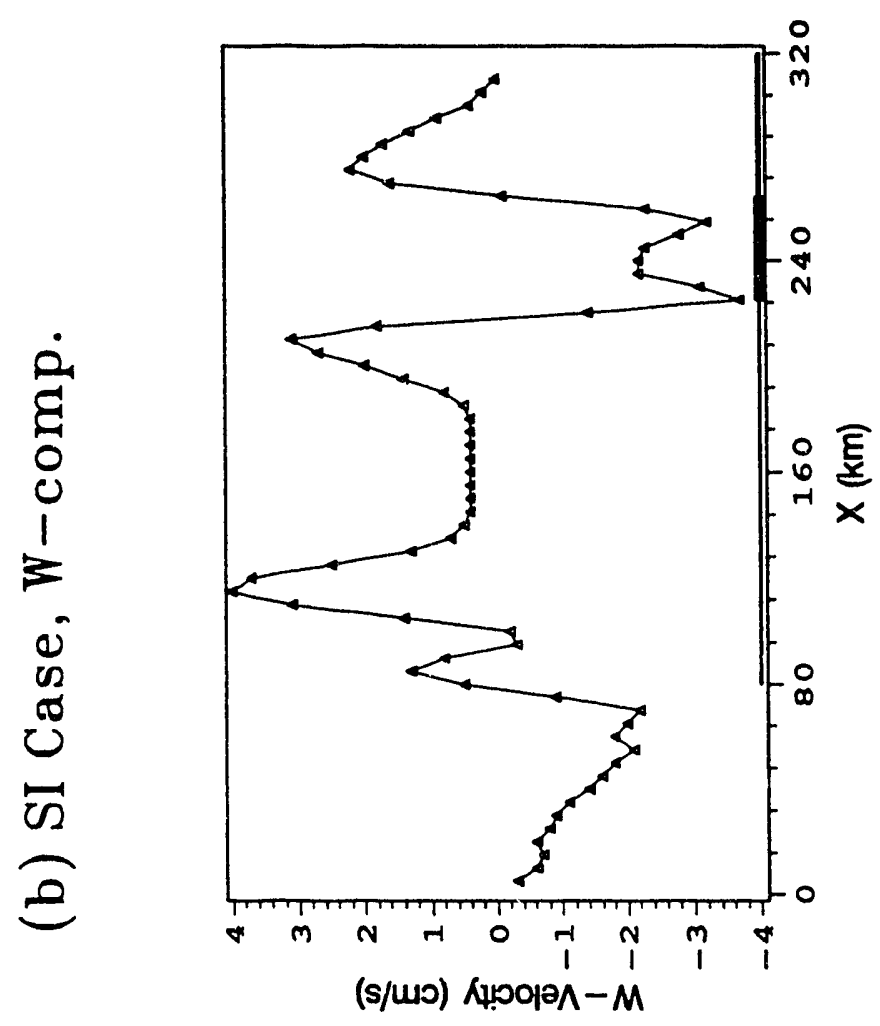

เั

$\stackrel{0}{0}$

는

30

要

웅웅

ळ

。

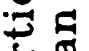

(1) 0

웅

e

옳웡

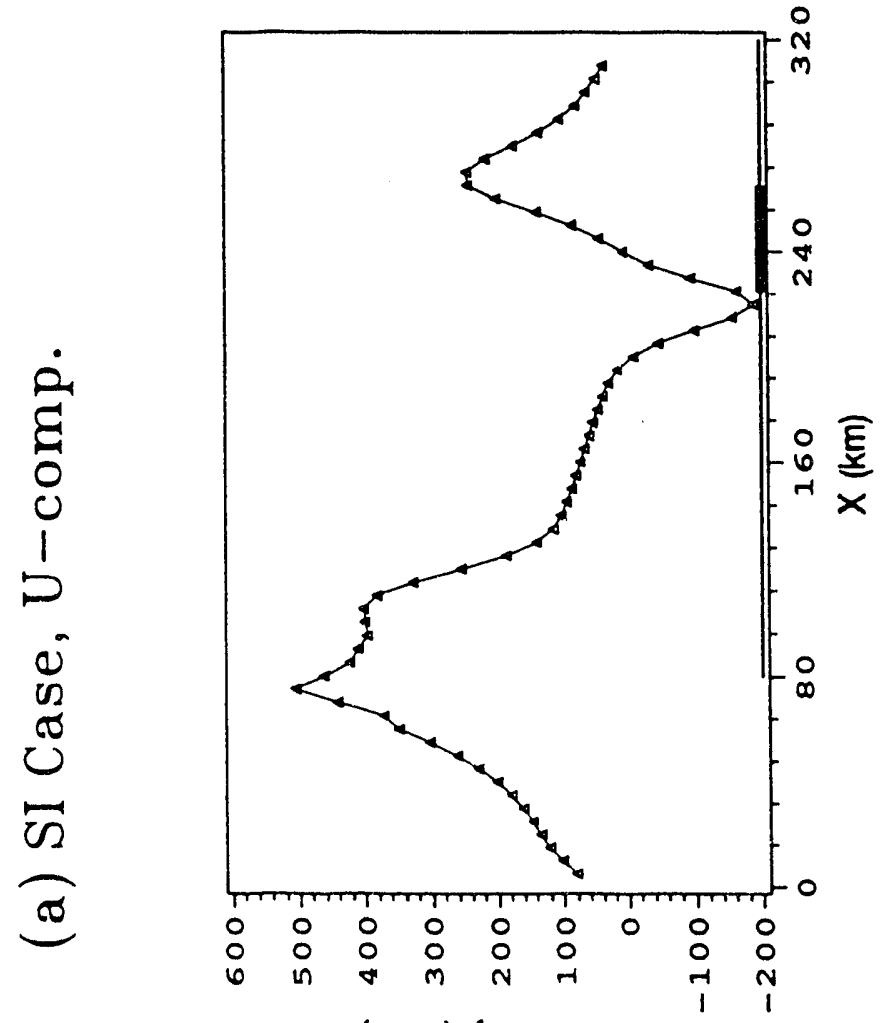

ธี 동

凷

동 क

ง.ำ

急

동

$\oplus$

๑

뎡로 몽

$\circ$ 잉

a

क

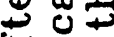

\& 0

$\ddot{0}$

ஸ름ㅁㅁㅁ

ฝัซ

bo :

理車。

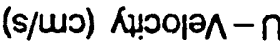



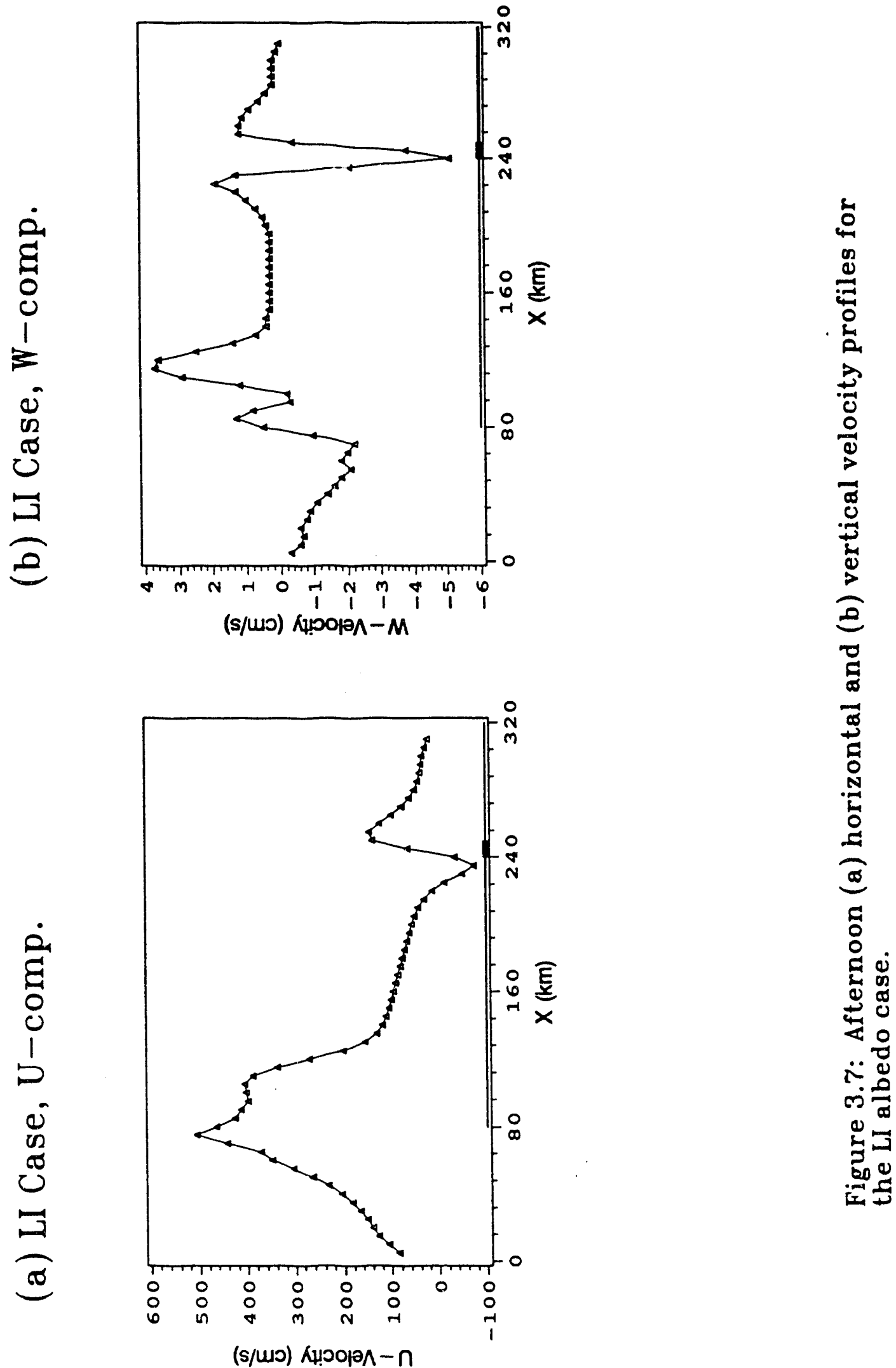

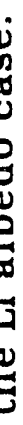




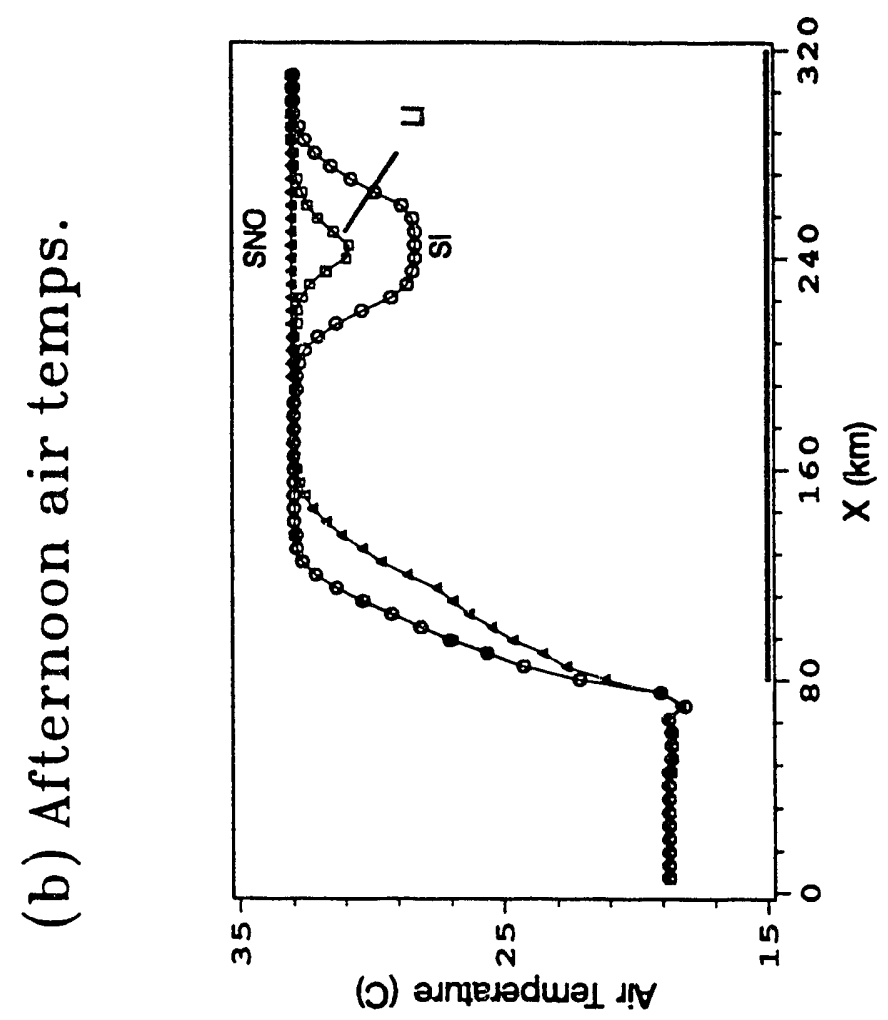

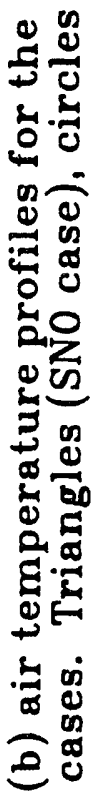

동옹

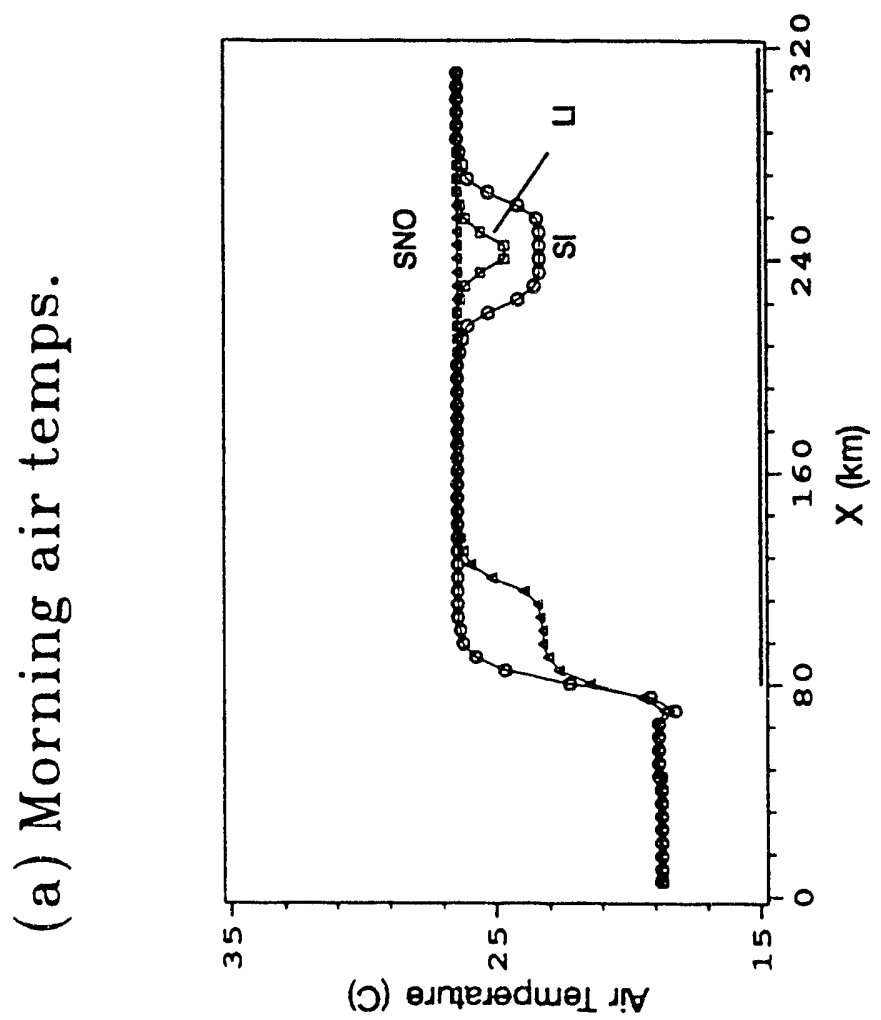

웅

엉

\%

-

두

ธ

๘

०० बै

สี \&

:

농

क ๙

ஸे

0

5.00

a du 


\section{CHAPTER 4 \\ Accurate Representation of the Urban Surface}

\section{DATA REQUIREMENTS}

In meteorological modeling studies, detailed surface characterizations are desirable since it is the heterogeneity of the surface which is responsible for many aspects of mesoscale circulations. At the very least, these models require a single domain-wide value for each of the surface characteristic parameters; albedo, roughness, moisture availability, vegetative fraction, substrate density, substrate specific heat capacity, and substrate thermal conductivity. Until recently, it has been common for researchers to specify domain-wide values of these parameters, neglecting their spatial variability. The two dimensional sensitivity study presented in the previous chapter has demonstrated the significant impact that variable surface characteristics can have on mesoscale meteorology. Other two-dimensional studies have reinforced the belief that accurate surface characterization is of utmost importance in simulating highly heterogeneous regions, eg. cities. There has been some recent work which suggests that the impact in three dimer.jions is somewhat reduced. Specifically, Ulrickson (1992) showed that use of variable surface characteristic data instead of domain-wide values resulted in only minimal impact on the local winds, even very near the surface. It is important to note, however, that Ulrickson's study did not address the impact in terms of air temperatures, which is one major focus in the present work. 
There are several issues that need to be investigated in the context of surface characterization. First of all, the method of obtaining the surface characterization can follow either of two approaches; inference from land-use data, and direct calculation from measured data. The second issue is that of scale. It is possible, for example to estimate moisture availability on any number of length scales ranging from the grid cell dimensions down to a region which is only a fraction of the size of the grid cell. Once a characteristic scale is chosen, the important problem is determination of how to include these data in a gridded representation of the domain surface. These issues are the focus of the following sections.

\section{LAND USE DATABASES}

There are several types of land use databases available to researchers. One such database is the United States Geological Survey Land Use and Development Area (LUDA) maps. These maps contain 50-m resolution for 26 categories of land coverage. Since these maps were developed to provide detailed surface data for the entire United States, the land use categories are quite broad, and a given mesoscale domain can only be divided into a small number of these categories. A major drawback of LUDA maps for use in urban climatology studies is the limited number of categories devoted to urbanized areas.

Urban climate researchers have developed several improved land coverage categorizations for use in urban settings. One notable characterization is that generated by Hjelmfelt et al. (1982). Hjelmfelt's study used 12 categories to describe the complex urban terrain of the St. Louis metropolitan area. Valley Research Corporation generated a similar database utilizing 23 categories to describe the land use in the greater Los Angeles Basin (Horie et al. 1990). 
By themselves, land-use databases are not useful for meteorological modeling purposes, since they offer information regarding only land use composition. In order to make these data useful some assumptions must be made. It is plausible to assume that regions containing similar land use patterns have similar surface characteristics. In order to generate surface characteristic data from landuse maps one can assume that there is a single value of each characteristic associated with each land use category. Aggregated values of each surface characteristic in a grid cell are derived by taking the area-weighted average of the land-use values. Table 4.1 summarizes the land-use categories from the Valley Research Corporation study and the associated surface characteristic values which have been obtained through a review of the existing literature. The range of albedo values presented in this table are from a low of 0.12 in recent industrial areas to a high value of 0.20 for various undeveloped categories. The corresponding extremes in roughness length are $0.05 \mathrm{~m}$ for desert and $1.25 \mathrm{~m}$ for city core. While some references suggest that this is a conservative estimate for the roughness of the city core, many hydrostatic models (including the CSUMM) have difficulty with large roughness elements. For moisture availability the extreme values are from a dry value of 0.01 for the desert up to a value of 0.35 for agricultural areas. This table also includes a list of peak daily values of anthropogenic heating and vegetative fraction associated with each land use. While the surface characteristics have been compiled from a large list of sources, the anthropogenic and vegetative fraction values were developed as plausible first-order estimates for the various land-use categories. The peak anthropogenic heating of 75-80 W $\mathrm{m}^{-2}$ for city cores and industrial regions is within the bounds of what other researchers have estimated for highly industrial cities (Oke 1987; Kimuia and Takahashi 1991). The urban and rural vegetative fraction ranges from 0 to $75 \%$ 
which is reasonable based on data from previous studies (Akbari et al. 1992).

Table 4.1: Land-Use Categories and Surface Characteristics ${ }^{\dagger}$

\begin{tabular}{|l|ccccc|}
\hline Land Use & Albedo & $\begin{array}{c}\text { Roughness } \\
\text { Length }(\mathrm{m})\end{array}$ & $\begin{array}{c}\text { Moisture } \\
\text { Availability }\end{array}$ & $\begin{array}{c}\text { Anthropogenic } \\
\text { Heat W } \mathrm{m}^{-2}\end{array}$ & $\begin{array}{c}\text { Vegetative } \\
\text { Fraction }\end{array}$ \\
\hline Close-set houses & 0.16 & 0.35 & 0.05 & 20.0 & 0.05 \\
Open-set houses & 0.15 & 0.30 & 0.10 & 15.0 & 0.25 \\
Close-set apartments & 0.16 & 0.40 & 0.03 & 20.0 & 0.05 \\
Open-set apartments & 0.15 & 0.35 & 0.05 & 20.0 & 0.10 \\
Admin/Cultural & 0.15 & 0.40 & 0.03 & 45.0 & 0.05 \\
City core & 0.14 & 1.25 & 0.03 & 75.0 & 0.00 \\
Recent Commercial & 0.14 & 0.50 & 0.05 & 50.0 & 0.00 \\
Old Commercial & 0.14 & 0.50 & 0.03 & 60.0 & 0.00 \\
Old Industrial & 0.14 & 0.60 & 0.03 & 75.0 & 0.00 \\
Recent Industrial & 0.12 & 0.60 & 0.05 & 80.0 & 0.00 \\
Freeway/infra. & 0.14 & 0.10 & 0.01 & 50.0 & 0.00 \\
Open areas (parks) & 0.20 & 0.15 & 0.30 & 2.0 & 0.50 \\
Wooded areas & 0.20 & 0.30 & 0.20 & 0.0 & 0.50 \\
Desert suburban & 0.20 & 0.10 & 0.03 & 5.0 & 0.00 \\
Agricultural & 0.20 & 0.10 & 0.35 & 0.0 & 0.75 \\
Grasslands & 0.20 & 0.05 & 0.20 & 0.0 & 0.00 \\
Sagebrush & 0.15 & 0.10 & 0.03 & 0.0 & 0.00 \\
Chramise Chaparral & 0.15 & 0.10 & 0.20 & 0.0 & 0.25 \\
Chaparral & 0.15 & 0.10 & 0.20 & 0.0 & 0.25 \\
Woodlands & 0.20 & 0.85 & 0.15 & 0.0 & 0.25 \\
Pinyon pine/Juniper & 0.20 & 0.85 & 0.15 & 0.0 & 0.50 \\
Forests & 0.18 & 1.00 & 0.20 & 0.0 & 0.50 \\
Desert & 0.15 & 0.05 & 0.01 & 0.0 & 0.00 \\
\hline
\end{tabular}

+ Based on data from Oke (1987), Sellers (1965), Threlkeld (1962), Kung et al. (1964), Taha et al. (1992), Wechsler and Glaser (1966), Stewart (1971), Monteith and Szeicz (1961), Hjelmfelt (1982), Seaman et al. (1989), and Monteith (1973). 
Table 4.2 lists the corresponding values of substrate properties. The ranges for each of these parameters are more confined than those for the surface characteristics. Density ranges from a minimum of 1.50 to a maximum of $2.40 \mathrm{~g} \mathrm{~cm}^{-3}$, diffusivity ranges from 0.0028 to $0.0057 \mathrm{~cm}^{2} \mathrm{~s}^{-1}$, and specific heat ranges from 0.92 to $1.84 \mathrm{~J} \mathrm{~g}^{-1} \mathrm{~K}^{-1}$. In each case, the total range does not much exceed a factor of 2.

Table 4.2: Land-Use Categories and Substrate Characteristics ${ }^{\dagger}$

\begin{tabular}{|l|ccc|}
\hline Land Use & $\begin{array}{c}\text { L ensity } \\
\mathrm{g} \mathrm{cm}^{-3}\end{array}$ & $\begin{array}{c}\text { Diffusivity } \\
\mathrm{cm}^{2} \mathrm{~s}^{-1}\end{array}$ & $\begin{array}{c}\text { Specific Heat } \\
\mathrm{J} \mathrm{g}^{-1} \mathrm{~K}^{-1}\end{array}$ \\
\hline Close-set houses & 1.50 & 0.0028 & 1.00 \\
Open-set houses & 1.60 & 0.0031 & 1.67 \\
Close-set apartments & 1.50 & 0.0030 & 0.92 \\
Open-set apartments & 1.60 & 0.0036 & 1.46 \\
Admin/Cultural & 1.60 & 0.0021 & 1.26 \\
City core & 1.60 & 0.0026 & 1.00 \\
Recent Commercial & 1.60 & 0.0042 & 1.26 \\
Old Commercial & 1.60 & 0.0042 & 1.26 \\
Old Industrial & 1.60 & 0.0052 & 1.00 \\
Recent Industrial & 1.60 & 0.0052 & 1.00 \\
Freeway/infra. & 1.60 & 0.0052 & 1.00 \\
Open areas (parks) & 2.40 & 0.0047 & 1.84 \\
Wooded areas & 2.00 & 0.0057 & 1.46 \\
Desert suburban & 2.00 & 0.0050 & 1.26 \\
Agricultural & 2.40 & 0.0038 & 1.84 \\
Grasslands & 2.00 & 0.0050 & 1.67 \\
Sagebrush & 2.00 & 0.0050 & 1.26 \\
Chamise Chaparral & 2.20 & 0.0026 & 1.46 \\
Chaparral & 2.20 & 0.0026 & 1.46 \\
Woodlands & 2.00 & 0.0057 & 1.46 \\
Pinyon pine/Juniper & 2.00 & 0.0057 & 1.46 \\
Forests & 2.00 & 0.0057 & 1.46 \\
Desert & 2.00 & 0.0050 & 1.26 \\
\hline
\end{tabular}

+ Based on data from Oke (1987), Sellers (1965), Threlkeld (1962), Kung et al. (1964), Taha et al. (1992), Wechsler and Glaser (1966), Stewart (1971), Monteith and Szeicz (1961), Hjelmfelt (1982), Seaman et al (1989), and Monteith (1973). 
Surface characterizations made using an urban terrain zone data base and an assumed relationship between land-use and surface characteristics may be faulty for the following reason. Many of the values found in the literature were obtained for areas in the mid-western United States, and may not be appropriate for use in southern California, with its distinct architectural differences. In addition, this method assumes that all areas of the same terrain zone type are identical in terms of the various surface characteristics. Clearly this is an oversimplification. For instance, the city core of Los Angeles probably has a much higher roughness value and lower moisture value than the city core of Long Beach. Similarly, each terrain zone type will have varying properties throughout the domain in question. In many cases this variation may prove difficult to evaluate. As will be discussed below, satellite remote-sensed data offer a partial solution to these problems.

\section{REMOTELY-SENSED RADIOMETRY DATA}

While land use data provide a noted improvement over the simple use of domain-wide surface characteristics, the accuracy of the land-use method is suspect for reasons outlined above. An area of research which appears to be quite promising with respect to improving surface characterization involves the use of remotely-sensed radiometric images from aircraft and satellite sensors. The general approach is that, with a knowledge of the incoming solar spectrum and the intervening atmosphere, researchers can estimate certain surface characteristics through analysis of the radiation sensed by high-altitude platforms.

Useful radiometric data are available from a number of sources. NASA flies ER-2 (U2) and C-130 aircraft which obtain Thematic Mapper Simulator (TMS) data. In addition, there are the satellite-based instruments such as the Advanced 
Very High Resolution Radiometer (AVHRR) and Thematic Mapper (TM). Each of these platforms provides measurements in a variety of wavelength bands. Various methods have been devised over the years to use these data to evaluate surface moisture availability, albedo, and vegetative cover. These methods will be outlined briefly in the following sections.

\subsection{Albedo}

Remotely sensed satellite data may be used in conjunction with a model for atmospheric attenuation to determine the albedo of a given area with a fair amount of accuracy (Brest and Goward 1987). The AVHRR satellite instrument provides radiance counts for a number of wavelength channels spanning the spectrum of solar radiation. One can obtain a very good analytic estimate of the incoming solar radiation at the outer edge of the earth's atmosphere. In addition, programs have been developed to estimate the transmission of solar radiation through the atmosphere. The satellite data allow estimation of the amount of solar radiation which is reflected from the surface of the earth and transmitted through the atmosphere. Using this information and the calculated incoming solar radiation, an accurate representation of the albedo of an area can be developed. The whole process can be calibrated and validated by analyzing large areas of well-known albedo such as the ocea.. surface or deserts. The resolution of the AVHRR data is on the order of $1 \mathrm{~km}$ which indicates that this approach may be easily applied to the scale of $5 \mathrm{~km}$, which is typical in mesoscale studies. 


\subsection{Vegetation}

Radiometer data are also useful in determination of healthy vegetation. A distinct characteristic of healthy vegetation is that it reflects very significant amounts of near-infrared radiation. It is therefore possible to develop a method whereby a single satellite image will provide an index representing the fraction of healthy vegetation coverage in each grid cell. Forster (1983), Goward et al. (1985), and Honjo and Takakura (1986) are among the growing field of researchers who have successfully obtained vegetative cover from radiometric data. Whild this method does not produce information on vegetation height, leaf area index, and a number of other important parameters, it may be sufficient for use in a simple parameterization, producing a rough estimate of the impact of vegetation on urban meteorology.

\subsection{Surface Moisture Availability}

Methods of using remote sensing to infer surface moisture availability have also been under development for several decades. Schmugge (1978) was among the first to propose and successfully carry out such analysis. These analyses generally rely on the highly negative correlation between surface temperature and surface moisture availability. Through analysis of the long-wave channels of radiometric data one can predict surface temperature and then infer moisture availability differences. In addition, the reflectivity of soil is generally a strong function of moisture content and may be used to generate an estimate of surface moisture availability, provided some information regarding soil type is available. 


\section{SURFACE CHARACTERISTIC PARAMETERIZATION}

The land-use and satellite methods for obtaining surface characteristic data are useful, but not complete. There are two remaining issues regarding the application of these data for use in meteorological models.

\subsection{The Issue of Scale}

In many modeling efforts the nominal length scales of the available surface characteristic data are much finer than the scales of the model grid cells. The problem of how to parameterize these high-resolution surface data for use in lower resolution computational grids is an important issue currently receiving a great deal of research attention. This is particularly true in the case of General Circulation Models (GCMs) with grid cell length scales on the order of $200 \mathrm{~km}$ (Dcran et al., 1992; Avissar and Pielke, 1989).

Consider a model grid cell encompassing an area $A$, which is subdivided into a finite number of subregions $A_{i}$. Suppose that values of a surface parameter $\phi_{i}$ corresponding to each one of these subregions are known. An average value $\bar{\phi}$ for the area $A$ is required which adequately represents the subgrid scale variations in $\phi$. The obvious first choice is to simply use algebraic area averaging:

$$
\bar{\phi}=\sum_{i=1}^{n}\left[\phi_{i} A_{i}\right]
$$

It can be shown that this method is appropriate for albedo and moisture availability, resulting in a conservation of latent and sensible fluxes across the grid cell. The result for surface roughness, however, is quite different (Taylor, 1987). There are several reasonable approaches for averaging of surface roughness. The surface roughness, $Z_{o}$ arises from the empirical definition of the friction velocity, $U^{*}:$ 


$$
u=\frac{U^{*}}{k_{0}} \operatorname{Ln}\left[\frac{\left(\mathrm{Z}-\mathrm{Z}_{d)}\right.}{\mathrm{Z}_{0}}\right]
$$

In this relation $U$ is the measured horizontal velocity, $U^{*}$ is the friction velocity, $k_{o}$ is the von Karman constant (0.4); $Z$ is the distance from the surface, $Z_{d}$ is the displacement height; and $Z_{0}$ is the empirically-derived roughness length. In practice, measurements of $U$ at varying values of $Z$ are used to evaluate the displacement height and friction velocity. If one wishes to define a grid cell value of velocity which represents the area average of the velocity of each subregion the result is:

$$
\bar{U}=\sum_{i=1}^{n}\left[\frac{A_{i} U^{*}}{k_{o}} \operatorname{Ln}\left[\left(Z-Z_{d}\right) / Z_{o}(i)\right]\right] .
$$

This assumes that the displacement height and the friction velocity are independent of $i$. Then, defining $\overline{Z_{o}}$ based on the following desired relationship,

$$
\bar{U}=\frac{U^{*}}{k_{o}} \operatorname{Ln}\left[\left(Z-Z_{d}\right) / \overrightarrow{Z_{o}}\right],
$$

and equating relation (4.3) to (4.4) one obtains the requirement that

$$
\overline{Z_{o}}=\prod_{i=1}^{i=n}\left[Z_{o}(i)^{\left(A_{i}\right)}\right]
$$

This method yields a grid-averaged roughness length scale which corresponds to an algebraic average of the friction velocity over the grid, and has been in fairly common use for several decades (Kung, 1963; Vihma and Savijarvi, 1991). The physical basis for this method of averaging, however, is somewhat uncertain since the empirical definition of roughness length has been developed over homogeneous terrain. 
An improved averaging technique for roughness length may be developed by considering conserved quantities. For instance, integrated energy flux is a conserved quantity. The algebraic sum of area-integrated energy flux (Watts) from each constituent subregion in a domain is precisely equal to the total flux from that domain. Starting with this approach then, the usual expression for sensible heat flux is:

$$
Q_{S}=\rho C_{p} U^{*} \Theta^{*}
$$

Here $\rho$ is the density of the substrate, $C_{p}$ is the specific heat capacity, $U^{*}$ is the friction velocity, and $\Theta^{*}$ is the "flux temperature" which is representative of the driving temperature difference between the surface and the air. The term $U^{*} \Theta^{*}$ in the above equation is generally prescribed based on empirical data. Within any model implementation, this relationship has a different functional form generally depending upon the value of the gradient Richarson number $R i$. The Richardson number represents the ratio between buoyant and shear production of turbulent kinetic energy and is given by the relationship:

$$
R i=\frac{g}{\Theta} \frac{\frac{\partial \Theta}{\partial z}}{\left[\left(\frac{\partial U}{\partial z}\right)^{2}+\left(\frac{\partial V}{\partial z}\right)^{2}\right]} .
$$

The functional form for $U^{*} \Theta^{*}$ used by Pielke in the CSUMM depends upon stability. When the Richardson number is negative (daytime), the expression is:

$$
U^{*} \Theta^{*}=1.35\left[\frac{k}{\operatorname{Ln}\left(Z / Z_{o}\right)}\right]^{2} \frac{(U+1)\left(\Theta_{p}-T_{1}\right)[-9.4 R i]}{1+5.3\left[\frac{k}{\operatorname{Ln}\left(Z / Z_{o}\right)}\right] 2\left[9.4 \sqrt{Z / Z_{o}}\right)}
$$

If the analysis is restricted to the case in which each of the variables which appear in (4.8) is constant across the domain with the exception of $Z_{o}$ one may 
write (4.8) as:

$$
u^{*} \Theta^{*}=\frac{f_{1}\left(Z_{0}\right)}{1+f_{2}\left(Z_{0}\right)}
$$

This expression may be analyzed at the extreme values of the functions. If, for instance, $f_{2} \ll 1$ then,

$$
U^{*} \Theta^{*} \propto \frac{1}{\left[\operatorname{Ln}\left[Z / Z_{0}\right]\right]^{2}}
$$

In the opposite case, when $f_{2} \gg 1$ the appropriate expression becomes,

$$
U^{*} \Theta^{*} \propto \sqrt{Z_{o} / Z} \text {. }
$$

These two limiting cases rely only on the value of $Z / Z_{0}$. The function $f_{2} r^{2} \Omega$ be estimated by polynomial expansion of the logarithmic term. The result is that $f_{2}$ varies as $Z / Z_{0}{ }^{3 / 2}$. Thus, (4.10) is the limiting case for small $Z / Z_{0}$, or relatively large roughness length. Equation (4.11) is the opposite case. The expression for $U^{*} \Theta^{*}$ used in the positive Richardson number case (evening, stable hours) is of the following form:

$$
U^{*} \Theta^{*} \propto \frac{1}{\operatorname{Ln}\left[Z / Z_{0}\right]}
$$

Each of these three expressions (4.10), (4.11), and (4.12) can now be used to develop an averaging method for $Z_{0}$. Using the concept of conserved sensible heat flux over the grid, the general relationship is:

$$
Q_{S}=\sum_{i=1}^{n} A_{i} Q_{S}(i)=\sum_{i=1}^{n} A_{i} \rho C_{p} U^{*} \Theta^{*}
$$

But since $\rho$ and $C_{p}$ are assumed constant, this relationship reduces to the requirement that $U^{*} \Theta^{*}$ evaluated using the grid-averaged roughness length is equal to 
the linear area average of $U^{*} \Theta^{*}$ in each subregion,

$$
\left[U^{*} \Theta^{*}\right]_{\left(\bar{Z}_{o}\right)}=\sum_{i=1}^{n} A_{i}\left[U^{*} \Theta^{*}\right]_{\left(Z_{o}(i)\right)} .
$$

The resulting expressions for each of the three cases above $(4.10,4.11$, and 4.12$)$ can then be solved for $\overline{Z_{0}}$. Equations 4.10 and 4.12 yield similar resillts. Application of (4.14) to (4.10) yields:

$$
\overline{Z_{0}}=\frac{Z}{\exp (\mu)}, \text { where } \mu=\left[\sum_{i=1}^{n} \frac{A_{i}}{\left(\operatorname{Ln} \frac{Z}{Z_{0}(i)}\right)^{2}}\right]^{-1 / 2} . \quad \text { (FA1, 4.15 }
$$

This result is equivalent to that used by Wieringa (1986) and van Dop (1983). Wieringa generated this relation by calculating grid-square averages of the local drag coefficients. This results in the assumption that wind speeds are constant from one homogeneous subregion to the next. Similarly, when (4.14) is applied to $(4.12)$ the result is:

$$
\overline{Z_{0}}=\frac{Z}{\exp (\mu)}, \text { where } \mu=\left[\sum_{i=1}^{n} \frac{A_{i}}{\operatorname{Ln} \frac{Z}{Z_{o}(i)}}\right]^{-1} \text {. }
$$

This result is equivalent to one developed by Andre and Blondin (1986). The result for (4.11) is much simpler expression:

$$
\overline{Z_{o}}=\left[\sum_{i=1}^{n} A_{i} \sqrt{Z_{o}(i)}\right]^{2} .
$$

Five possible expressions for averaging $Z_{o}(i)$ have been presented. Researchers have developed other expressions based on varying theoretical assumptions. In addition to analytic approaches, the use of mesoscale modeling to develop roughness parameterizations based on predicted wind profiles has shown some 
promise (Vihma and Savijarvi, 1991). Three of the expressions presented here were based on the conservative heat flux method, with differing assumptions regarding atmospheric stability and variables within the heat flux parameterization. These three schemes are given by (4.15), (4.16), and (4.17). These methods will be referred to as Flux Average 1, 2, and 3 (FA1, FA2, FA3). The simple Algebraic Average (AA) of (4.1) and the Geometric Average (GA) of (4.5) are the other two methods.

In order to test the potential differences in $\bar{Z}_{o}$ produced by each method, the following investigation was conducted. First, it was assumed that in a given region, the roughriess values for each subregion $\left(Z_{o}(i)\right)$ are uniformly distributed between a maximum, $Z_{\max }=1.5 \mathrm{~m}$ and a minimum, $Z_{\text {mir }}=0.03 \mathrm{~m}$. If a region is composed of $n$ subregions, $n$ samples were uniformly extracted from the distribution and each of the averaging techniques was applied to this concocted highresolution data set. This process was repeated 10,000 times with different random seeds to obtain an ensemble average for each method. The resulting gridaveraged roughness values are plotted in Figure 4.1. This figure illustrates the significance of choice of averaging method. Each averaging method results in a prediction of $\overline{Z_{0}}$ which asymptotically approaches a constant value as the number of subregions is increased. Based on the sampling range of 0.03 to $1.5 \mathrm{~m}$, the simple algebraic averaging scheme, AA is expected to yield an average of $0.765 \mathrm{~m}$. This is precisely the asymptotic value shown for AA in this figure $( \pm$ $0.07 \%$ ). Each of the flux averaging schemes resulted in a predicted roughness value of 0.68 to $0.71 \mathrm{~m}$, which is about $10 \%$ lower than the AA value. The roughness prediction from the GA scheme was $0.60 \mathrm{~m}$, which is $22 \%$ lower than the AA value of $0.765 \mathrm{~m}$. This large range of predicted roughness values indicates that the choice of averaging technique can yield significant variations in 
roughness values to be used in modeling a particular region. Which method is best? This can be a very difficult question to answer. Since heat and momentum transport correlations are derived for homogeneous terrain, there is no obvious averaging technique for heterogeneous terrain. The conservation-based averaging schemes have a strong physical basis, and are therefore recommended. Each of the flux average schemes was developed subject to some constraints. For this reason, FA1, FA2, and FA3 are each valid only under particular meteorological conditions (daytime, evening, low wind speed days, etc.). Since each of these schemes yields a similar result, however, it is reasonable to simply take the scheme which yialds the middle value and use it without discretion. For this reason, FA3 (4.17) is the recommended choice, especially if one is most concerned with accurate prediction of the surface heat fluxes and low-level air temperatures. FA3 also happens to be the simplest expression of the three flux averages. If, on the other hand, accurate prediction of wind fields is more important it is suggested that the geometric average of (4.5) be used.

In either case, the discrepancy between the simple algebraic average and either of the other methods is relatively small when viewed in light of the model's mild sensitivity to surface roughness. Furthermore, if one uses the averaging techniques just presented with (4.2) to back out a vertical velocity profile for each approach, it can be shown that a constant velocity oftset will arise. This offset is equal to $\frac{U^{*}}{k_{o}}\left(\operatorname{Ln}\left(\frac{Z_{o, i}}{Z_{o, j}}\right)\right)$ where method $i$ is being compared with method $j$. For the extreme comparison of the Algebraic Average and the Geometric Average, with a nominal friction ve'.ucity of $1 \mathrm{~m} \mathrm{~s}^{-1}$, this offset between the velocity profiles is equal to $0.58 \mathrm{~m} \mathrm{~s}^{-1}$. 
The assumptions made in various aspects of this analysis dictate that the results be viewed as preliminary. In going from (4.8) to (4.9) it was assumed that bulk velocity, temperature, and Richardson number are all independent of location in the domain. While this is clearly in error, it was necessary so that the effect of variable surface roughness could be isolated. In actuality, air temperature in a $5 \mathrm{~km}$ grid cell commonly varies by as much as $3^{\circ}$ to $5^{\circ} \mathrm{C}$. In the heterogeneous urban canopy the wind profiles and Richardson number may also vary wildly within a single grid cell. It is important to improve the fundamental understanding of subgrid scale aggregation of surface characteristics when developing parameterizations for heterogeneous terrain. Using existing parameterizations, however, it is unclear whether the different averaging methods will have much impact on simulated air flows. This is an area which requires further study. For the present research the simple algebraic average has been employed since the implications of the other averaging techniques are not yet fully understood.

\subsection{Parameterizing Vegetation}

Most vegetation parameterizations for use in meteorological models are complex requiring a great deal of information on vegetation characteristics in the computational domain. A typical parameterization method involves using an electrical analogy, where the latent and sensible heat fluxes from vegetated canopies are evaluated as functions of driving potentials and flux resistance terms. For each type of vegetation present, the modeler must specify terms which represent the resistance of that particular species to sensible and latent heat transfer. As an example, a typical implementation of the Taconet and VidalMadjar (1986) scheme requires specification of the inter-leaf airspace resistance 
for sensible heat flux $\left(r_{a f}\right)$, soil surface-canopy resistance for latent heat flux from the ground $\left(r_{a g}\right)$, and the leaf resistance to latent heat flux, $r_{l}$. Furthermore, parameterizations require that the model track leaf surface temperatures and evaluate the saturation vapor pressure at the leaf temperature. In addition, the density and distribution of vegetation are specified in terms of Leaf Area Index (LAI), Mean Vegetation Height (MVH), and, in cases of partial vegetative cover, the fraction of vegetated area, $f$. These parameters are used in the partitioning of latent and sensible fluxes as well as in the calculation of radiative exchange.

While some existing meteorological models explicitly incorporate a vegetation parameterization, others do not. At the present time there is a need for a simple method whereby models without a vegetation parameterization can be used to quickly assess the impact of vegetative modifications on the local meteorology in a particular application. Such a method is outlined below, but is only designed to capture first order effects and should be used only to provide preliminary assessments of vegetative impacts.

In implementing a vegetation parameterization, it is useful to first identify and understand the proposed application. In the present case, it was assumed that the current model, which has no explicit vegetation parameterization, yields a satisfactory representation of the meteorology in the computational domain. Since the potential meteorological and air-quality impacts of tree-planting programs are the primary concern, this study addresses the result of adding a given amount of urban vegetation to selected grid cells. It is reasonable to represent the tree-planting program as consisting of identical typical trees. Thus, many vegetative parameters are assumed constant for any vegetation in the domain. The only variable which must be introduced to the existing CSUMM model is the fractional vegetative cover, $f$, which ranges from 0.0 to 1.0 . 
The parameterization which is proposed here is an attempt to represent the impacts of vegetation through modification of the existing input parameters to the CSUMM. A model which includes an explicit vegetation parameterization may be thought of as an $(n+1)$-parameter model. The CSUMM, on the other hand, is an n-parameter model, lacking the vegetative fraction $f$ as an input variable. The goal here is to represent $f$ through modification in some or all of the available $\mathrm{n}$ parameters.

The first step in representing vegetation in the underparameterized model is to identify the parameters which will be modified to account for changes in $f$. The choice of parameters should rely upon some physical basis.

The addition of vegetation directly modifies the suriace albedo and roughness values. In addition, vegetation acts as a pump which extracts water from the root zone of the substrate layer and evaporates it from leaf surfaces. Thus vegetation acts similarly to surface moisture. Vegetation has a two-fold impact on sensible heat flux. First, since the addition of vegetation increases the latent heat flux, the surface is cooler, resulting in a lower driving force for sensible heat flux. On the other hand, the addition of vegetation increases the sensible heat transfer rate coefficient because of the heat transfer augmentation effects of the large area of leaf surfaces in the wind field.

The two-dimensional simulations discussed in Chapter 3 indicate that small surface roughness modifications have little impact on local meteorology. The application of any vegetation modification scheme would likely involve the planting of trees which are 2 to $4 \mathrm{~m}$ in height in neighborhoods that already have a surface roughness parameter influenced by structures which are of the same general dimensions. After years of growth, the impact of trees on local surface roughness may be important, but in the early stages of a tree planting program 
the direct impact on roughness length is expected to be small, and the resulting impact on meteorology should be negligible. Thus, roughness is not included in this parameterization.

Measurement studies have shown that the albedo of vegetation is generally around 0.20 . Thus, any addition of vegetation to a grid cell should be accompanied by an area-weighted change in the albedo of that grid cell. Since the albedo of the residential terrain types, as defined for the UTZ data base, were in the range of 0.15 to 0.18 , a vegetation modification scheme will generally result in a small increase in the albedo of the modified cells. For vegetation schemes which increase the areal vegetative coverage by 15 percentage points, the corresponding increase in albedo will be on the order of 1 percent. While this impact is small, it is easily included directly into the model input and does not need to be included in this parameterization. The appropriate modification to the albedo resulting from the addition of a given fractional cover of vegetation is given by:

$$
\rho_{S}^{\prime}(i, j)=\rho_{S}(i, j)+f *\left[0.20-\rho_{S}(i, j)\right]
$$

where $\rho$ and $\rho_{S}^{\prime}(i, j)$ are the original and modified values of albedo for grid cell $(\mathrm{i}, \mathrm{j})$.

The two-dimensional simulations revealed that substrate properties play only a minor role in the surface energy balance. They also have very little relationship to the changes in heat transfer processes resulting from vegetation. For these reasons, substrate properties are also excluded in the development of this simplified parameterization.

The latent heat flux resulting from surface moisture and from vegetation are very similar processes. They are generally both represented in terms of a 
potential for evaporation, and a resistance (or network of resistances) to this flux. In order to capture the impact of vegetation in terms of latent heat flux it is therefore necessary to include moisture availability, $\mathrm{m}$, in this parameterization.

\section{STATISTICAL PARAMETERIZATION (SP)}

The moisture availability has been identified as the single parameter to be used in representing vegetation in this new parameterization. Specifically, it is proposed that the value of moisture availability be modified in order for the model results to represent the impacts of a proposed vegetation augmentation.

As a starting point, an existing one-dimensional meteorological model which has a complete vegetation parameterization within it was obtained. The model is that of Carlson (1989) which includes a vegetation parameterization similar to Taconet and Vidal-Madjar (1986). The Carlson model allows input specification of numerous vegetative parameters. For the purposes of this simplified parameterization, however, the only vegetation variable of interest was the fractional vegetative cover $f$. The primary input parameters for the Carlson model then are the albedo $\rho_{S}$, the moisture availability $m$, surface roughness $r$, the substrate properties, and the fractional vegetation, $f$. The CSUMM model to which a vegetation capability was to be added, allows specification of the same input parameters with the exception of $f$. The goal, was to use the $(n+1)$-parameter Carlson model to develop a suitable parameterization such that an $\mathrm{n}$-parameter model captures the physics of the missing vegetation parameter through a suitable augmentation of the effective moisture value.

To quantify the error of this parameterization a generalized diagnostic variable was defined for each simulation. A value of $m^{\prime}$ was defined such that this error was minimized. Since the primary impact of vegetation is on the surface 
latent heat flux, a natural diagnostic variable would be a daily average or peak value of the latent heat flux term. Another useful diagnostic variable might be developed from the sensible heat flux or the near surface air temperature. The Carlson model generates output of surface fluxes and air temperature at any specified time interval. To develop appropriate diagnostic variables from these variables temporal averages were computed. The choice for any variable $D$, is the following daytime average:

$$
\hat{D}=\frac{1}{14} \sum_{i=10}^{16}\left[D_{1 / 2}\left(T_{i}\right)\right]
$$

The temporal average in this equation represents a discrete average of the vari- ble from 10:00 a.m. to 4:00 p.m. This term is evaluated using fourteen halfhourly data values $\left(D_{1 / 2}\right)$. There are three reasons for this choice of averaging technique. First, fluxes within this time frame have been demonstrated to be essentially linear functions of $f$, which simplifies the statistical modeling. Second, it is important to accurately represent vegetation during the hours of the day during which air temperatures and photochemical smog are greatest. Third, the Carlson model, as is the case with many models is less reliable during the evening and early morning hours.

The general approach was to conduct numerous simulations with the Carlson model. The results were then fitted with a statistical model, $\hat{D}(\mathrm{~m}, \mathrm{f})$. Due to the linearity observed in flux-parameter plots, and the desired simplicity of the parameterization, the model was chosen to be only linearly dependent upon each parameter. In the case of vegetative fraction, the relationship was allowed to be quadratic. The statistical model of the diagnostic variable was defined as follows:

$$
\hat{D}=A_{1}+B_{1} m+B_{2} f+C_{1} f^{2}
$$


When this statistical model was applied to the case with modified values of $m$, and with $f$ set equal to zero, the following expression for a model excluding explicit parameterization of vegetative fraction $f$ was obtained.

$$
\hat{D}^{*}=A_{1}+B_{1} m^{\prime}
$$

By relating $\mathrm{m}^{\prime}$ to $\mathrm{m}$, a two parameter statistical model can be collapsed into a single parameter model, which can then be used as an implicit parameterization of vegetation for the CSUMM. It is reasonable to express the relationship between the primed variable and the original variable as:

$$
m^{\prime}=m+\delta m(f) \text {. }
$$

Now, relating meteorological predictions for the underparameterized model to the predictions of the fully parameterized version, the error in terms of $\hat{D}$ is given by:

$$
\varepsilon=\left(\hat{D}-\hat{D}^{*}\right)=-B_{1} \delta m+B_{2} f+C_{1} f^{2} .
$$

To minimize the error in this parameterization, $\varepsilon$ is set to zero resulting in an equation for the functional form of $\delta m$ :

$$
\delta m=\frac{B_{2}}{B_{1}} f+\frac{C_{1}}{B_{1}} f^{2} .
$$

This expression represents the "effective" augmentation to be applied to the moisture availability to represent the effect of increasing vegetative cover.

In the original attempt to develop this parameterization, the statistical model was allowed to be a higher order polynomial function of $m$ and $f$. When the error expression was set to zero the result was a polynomial function of $\delta \mathrm{m}$. If the model was more than a quadratic function of $m$, then $\delta m$ would need to be solved for through a numerical minimization algorithm for each choice of $(m, f)$. 
This defeats the original goal of simplicity. In the case where the model was a quadratic function of $m$ the result was a quadratic expression for $\delta m$. While this expression can be explicitly evaluated, the roots may be complex under some circumstances, and the same numerical minimization algorithm is required, since a real root may not exist. For these reasons, the statistical model could be no more than a linear function of $m$.

Since the statistical model requires evaluation of 4 constan $s$, a sufficient number of simulations was required so that each constant could be evaluated. Reasonable ranges of $m$ and $f$ were defined: $0.01<\mathrm{m}<0.30$; and $0.00<\mathrm{f}<0.80$. Each range was then divided into 25 equal segments and numerical simulations were conducted for each of the resulting 625 pairs of $(m, f)$. Applying the above procedure to each of 4 diagnostic variables, 625 datum points were obtained, each of which had an associated value of the parameters $(m, f)$ and each diagnostic variable $(\hat{H}, \hat{E}, \hat{Q}, \hat{T})$. These diagnostic variables correspond to the temporal averages of the sensible heat flux, latent heat flux, sum of sensible and latent heat fluxes, and the near surface air temperature, respectively. Figure 4.2a demonstrates the positive correlation between $\hat{H}$ and $f$. The slope of the best linear fit through these computational datum points is $\mathbf{- 8 0 . 9}$. Figure $\mathbf{4 . 2 b}$ shows that the corresponding slope for $\hat{H}$ vs. $m$ is -252.1 . By dividing these two slopes the first (primary) term in (4.25) is found to be $\delta m \approx 0.32 f$. In other words, an increase of vegetation by 10 percentage points can be modeled by an augmentation of the moisture availability by 0.032 . This result is dependent, however, upon the choice of diagnostic variable for the analysis. Figure 4.3 shows the corresponding relationship for the latent heat flux diagnostic variable. Table 4.3 lists the results of applying the statistical model to each of the four diagnostic variables previously defined. 
Table 4.3: Statistical Model Parameterization Results

\begin{tabular}{|l|rrrr|c|}
\hline $\begin{array}{l}\text { Diagnostic } \\
\text { Variable }\end{array}$ & $A_{1}$ & $B_{1}$ & $B_{2}$ & $C_{1}$ & $\delta m=$ \\
\hline$\hat{H}$ & 277.63 & -252.14 & -80.87 & -5.00 & $0.32^{*} f+0.020^{*} f^{2}$ \\
$\hat{E}$ & 134.95 & 507.50 & 171.91 & 6.86 & $0.34^{*} f+0.014^{*} f^{2}$ \\
$\hat{Q}$ & 412.58 & 255.36 & 91.04 & 1.86 & $0.36^{*} f+0.007^{*} f^{2}$ \\
$\hat{T}$ & 28.96 & -4.97 & -1.02 & -0.45 & $0.21^{*} f+0.091^{*} f^{2}$ \\
\hline
\end{tabular}

Note that in each case, the quadratic term is very small and may be neglected. Based on these results it appears that a good simple parameterization for vegetation is to represent each $10 \%$ increase in vegetation by an increase of 0.034 in moisture availability. At the same time, however, the albedo should also be modified according to (4.18). These two modifications represent perhaps the simplest method by which the first order implications of vegetation modifications may be included in a meteorological model. It is not suggested here that this procedure can replace a full vegetation parameterization. Instead, it may be used as a diagnostic tool in determining the general implications of vegetation modifications in situations where an implicit vegetation parameterization is either not available, or difficult to implement effectively. It should also be emphasized that this parameterization was developed using simulations of a typical summer day at a specific latitude of $34^{\circ} \mathrm{N}$, and may be of only marginal applicability in significantly different situations.

\section{BULK LAYER PARAMETERIZATION}

To evaluate the performance of the above simple statistical parameterization, a slightly modified version of the bulk-layer parameterization (BLP) discussed by Avissar and Pielke (1989) was implemented. The parameterization was originally developed by Avissar and Mahrer (1988), and validated against measured data. In the BLP, the surface is assumed to consist of two layers, a 
vegetation layer and a soil layer. The energy balance for the vegetation layer is written as

$$
R_{N o}+E_{0}+H_{0}=0,
$$

where $R_{N v}$ is the net radiant flux on the canopy, $E_{v}$ is the latent flux from the vegetation, and $H_{0}$ is the sensible flux from the canopy. The energy balance for the soil layer contains one additional term:

$$
R_{N G}+E_{G}+H_{G}+S_{G}=0
$$

Here, $S_{G}$ is the ground heat flux term. In the BLP, Avissar defined the "flux temperature", which was a weighted average between the vegetation temperature and the ground surface temperature. In a similar fashion a weighted value for surface specific humidity was defined. The global values of latent and sensible heat flux were then evaluated based on these weighted temperature and humidity values. The same weighting function was then used to partition this global flux between the vegetation and the ground. The weighting function was defined as follows:

$$
\sigma_{f}^{\prime}=\frac{2 L A I \sigma_{f}}{1+2 L A I \sigma_{f}}
$$

where $\sigma_{f}$ is the fraction of surface covered by vegetation (also referred to as $f$ ) and LAI is the leaf area index (single surface area of leaves divided by the area covered by vegetation).

The radiative terms were evaluated separately, based on the equations for reradiation within the canopy. These equations rely upon $\sigma_{f}$ rather than $\sigma_{f}$ to partition radiative exchange. In the present study it was found that for values of $\sigma_{f}$ less than 0.10 the predicted vegetative temperature takes on unrealistically low values (below $280 \mathrm{~K}$ ). Since the predictions of the ground temperatures 
remain physical, the resulting predictic: of the weighted surface temperature was within reason. The explanation for this result is that, the partitioning of latent and sensible heat fluxes between the ground and vegetation is based on $\sigma_{f}$ while the radiative partitioning is based on $\sigma_{f}$. As an example, when LAI $=3$ and $\sigma_{f}=0.10$, the value of $\sigma_{f}$ is 0.375 . This results in $38 \%$ of the latent and sensible flux coming from the vegetation, but only (on the order of) $10 \%$ of the radiative flux coming into the vegetation canopy. This imbalance in energy flux results in a substantial cooling of the vegetation. Since the flux temperature and humidity are also weighted by $\sigma_{f}^{\prime}$, the sensible and latent fluxes respond slowly to the lowered vegetation temperature. Avissar and Pielke (1989) only presented vegetation temperature profiles for values of $\sigma_{f}$ ranging from 0.25 to 1.00 . Thus, it is unclear whether they would have encountered the same difficulty. In order to subtend this problem, $\sigma_{f}$ in Avissar's scheme has been replaced with $\sigma_{f}$.

The present scheme contains one more significant mod:fication to the Avissar BLP method. Avissar defined the dimensionless relative stomatal resistance

$$
d_{s r}=\frac{\left[d_{s m}+\left(d_{s M}-d_{s m}\right) * f_{R} f_{T} f_{V} f_{C} f_{\psi}\right]}{d_{s M}},
$$

where $d_{s m}$ and $d_{s M}$ are the minimum and maximum stomatal conductance. Each of the $f_{i}$ functions represent the influence of some environmental factor. The functionality of $f_{R}$ represents the impact of solar radiation and was the only factor directly incorporated in this model. Each of the other factors affecting stomatal conductance was assumed to be constant, amounting to a multiplicative factor of 0.70 . This value was chosen such that the model was capable of approximating test results found in Avissar and Pielke (1989). 


\section{COMPARISON OF VEGETATION PARAMETERIZATIONS}

Avissar and Pielke (1989) conducted a number of benchmark simulations with their bulk layer parameterization (BLP) and what they considered an improved Subgrid-scale Surface Forcing Parameterization (SSFP). The SSFP divided heterogeneous grid cells into $\mathrm{n}$ subregions of homogeneous content. Within each subregion, the BLP was used to predict surface fluxes. The grid cell fluxes were then determined by an area-weighted averaging of each of the $n$ subregions.

The benchmark simulations developed by Avissar are used here to compare the simple statistical parameterization and the current implementation of the BLP with the parameterization of Avissar. These simulations were conducted for a dry clay-loam soil at a latitude of $37^{\circ} \mathrm{N}$ on July 15 . Each simulation is initialized at 0600 LST and runs for at least 12 hours. The initial temperature of the surface is $300 \mathrm{~K}$ and the low level humidity is uniform (up to $1 \mathrm{~km}$ height) with a value of $10.0 \mathrm{~g} \mathrm{~kg}^{-1}$. For complete details of the initial conditions of the simulations, see Avissar and Pielke (1989).

The peak values of sensible and latent heat flux are given in Figure 4.4. In terms of sensible heat flux predictions the current implementation of the BLP is more accurate than the statistical parameterization (SP). It is interesting to note, however, that for latent heat flux predictions the SP is superior, especially for low values of modeled vegetative cover. The BLP, having a basis in plant physiology, and having been previously validated in monitoring studies is considered to be superior in general, and was permanently incorporated in the CSUMM for use in future simulations. 


\section{ANTHROPOGENIC HEATING}

In an attempt to include anthropogenic heating in models of the urban climate some researchers (Carlson 1990) suggest adding $Q_{f}$ to the surface energy balance. In reality, however, only a very small fraction of $Q_{f}$ goes directly into the substrate. The majority of $Q_{f}$ enters into the first several meters of air (eg. exhaust cars and machinery; heat exchange by air conditioners and heat pumps; and waste heat from other uses of electricity).

A simple 4-air layer energy balance model (SIMPLE) was developed to investigate the importance of accurate parameterization of $Q_{f}$. SIMPLE assumes perfect mixing within each of the four air layers. The air layers are 5, 25, 50, and 500 meters high. Heat transfer coefficients between each of the air layers and between the surface and the first air layer are specified. Transmittances for each air layer and additional parameters are specified as necessary. At each time step, the surface energy balance is evaluated followed by evaluation of the energy transfer to each of the air levels. The plausibility of results from SIMPLE were validated using data from a monitoring experiment in Sacramento California (Sailor et al. 1992).

In order to investigate the impact of the choice of partitioning of $Q_{f}$ on temperature predictions SIMPLE was run in two contrasting modes. In the first mode all $Q_{f}$ enters the substrate directly. In the second mode all $Q_{f}$ enters the 5 meter high air level directly. The results of these two cases are presented in Figure 4.5. It is evident that while the partitioning of a moderate level of $Q_{f}$ has a negligible impact on the surface temperature, it may be responsible for as much as a half degree Celsius error in near-surface air temperature predictions. As this figure illustrates, the partitioning of $Q_{f}$ into the air instead of the soil results in a half degree Celsius increase in air temperature and only a very minimal decrease 
in soil surface temperature. A better method, is to estimate the fraction of anthropogenic heat that enters the substrate directly, add this fraction to the radiative balance at the surface, and add the remaining anthropogenic heat directly to the air. Because the fraction of $Q_{f}$ which enters the substrate directly is very small, a reasonable approach is to simply model $Q_{f}$ as a heat source term in the first air layer of a meteorological model. This is precisely the assumption made in both the sensitivity study of Chapter 3 , and the full three-dimensional simulations of the following chapter. 

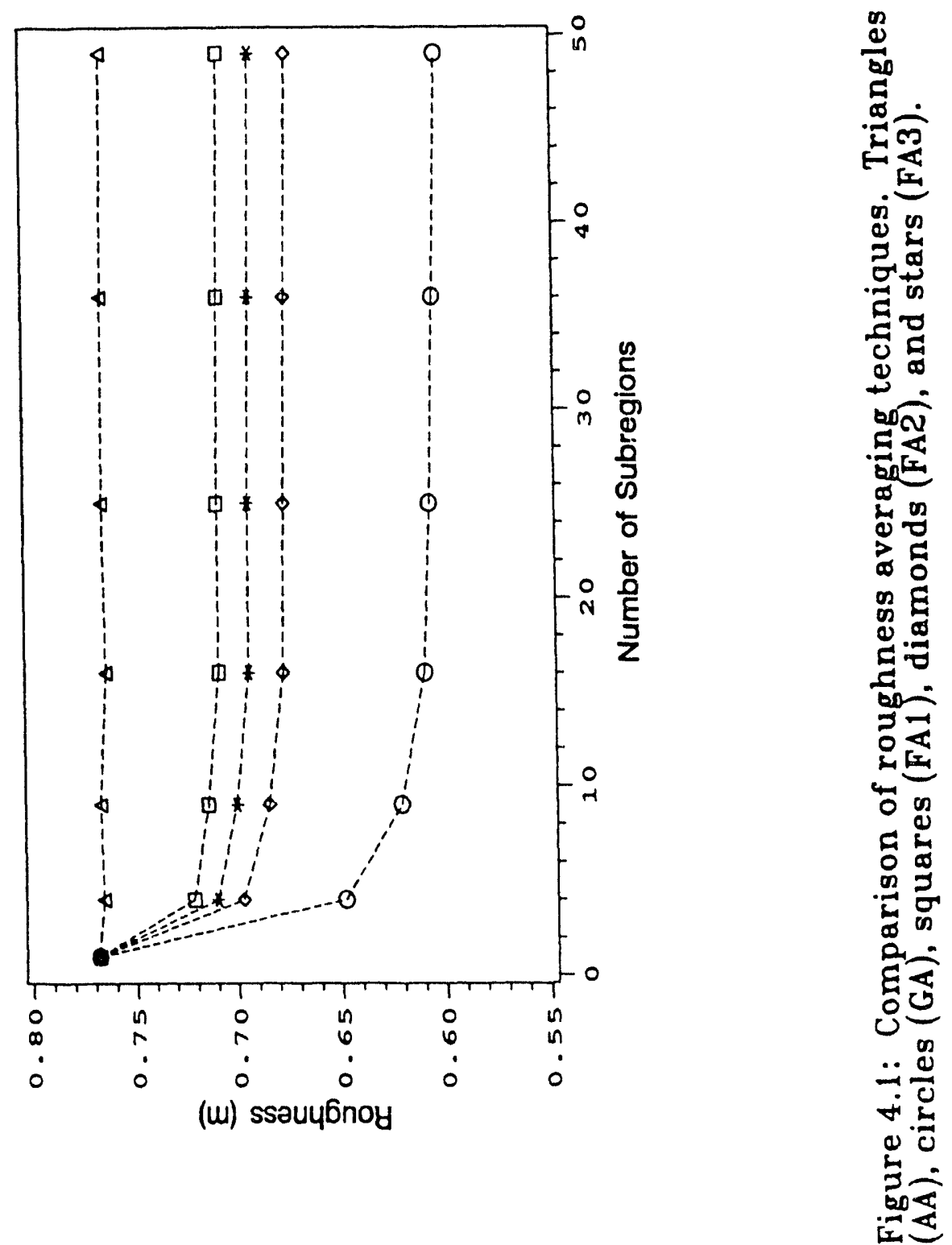


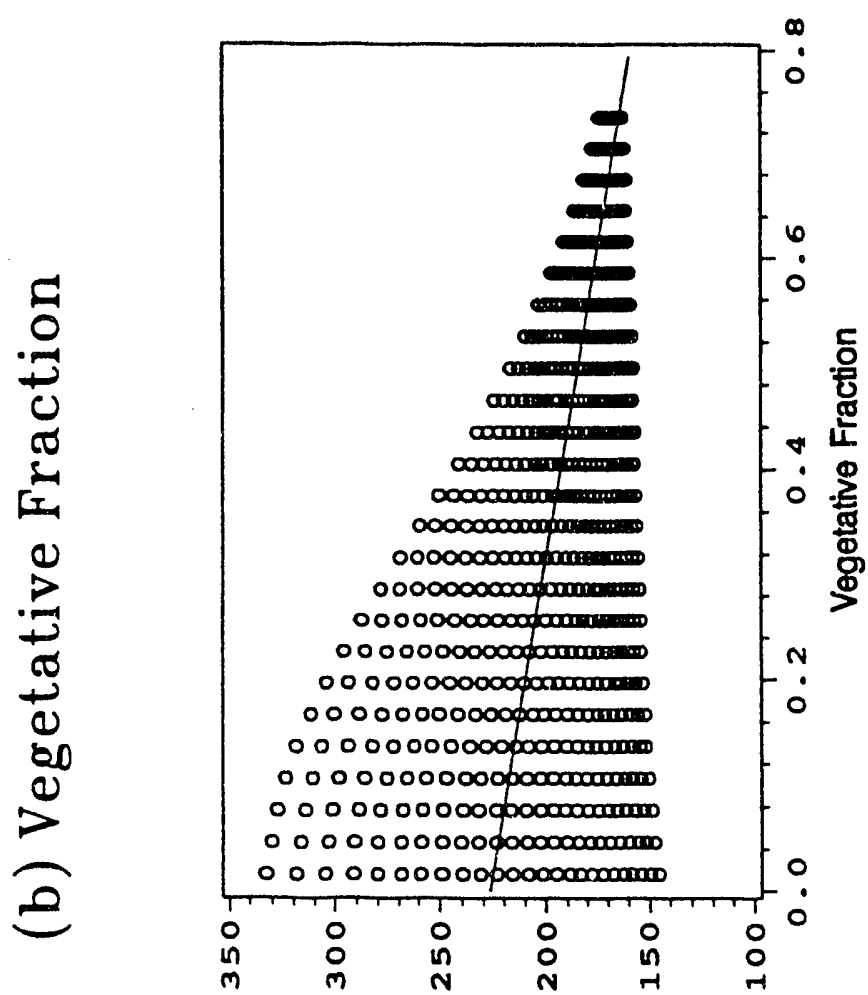

(u bs/smpa) xny ө|q!suas

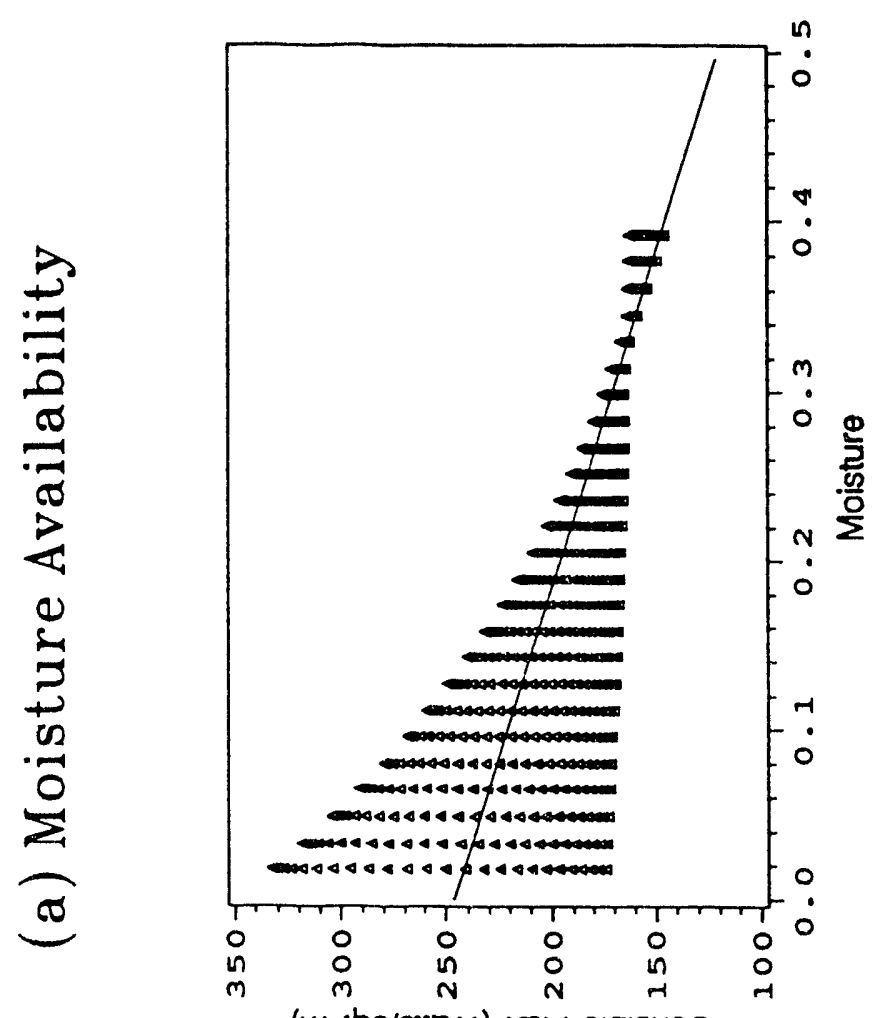

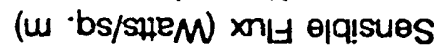




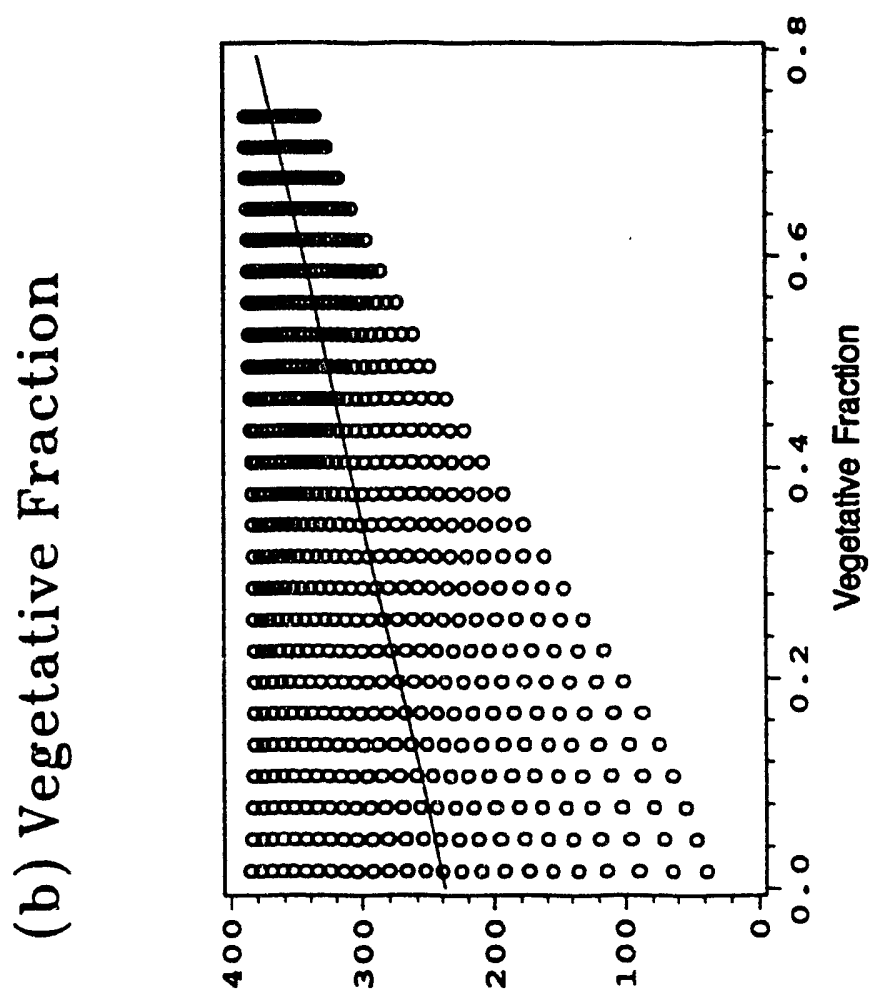

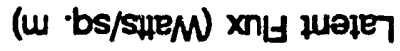

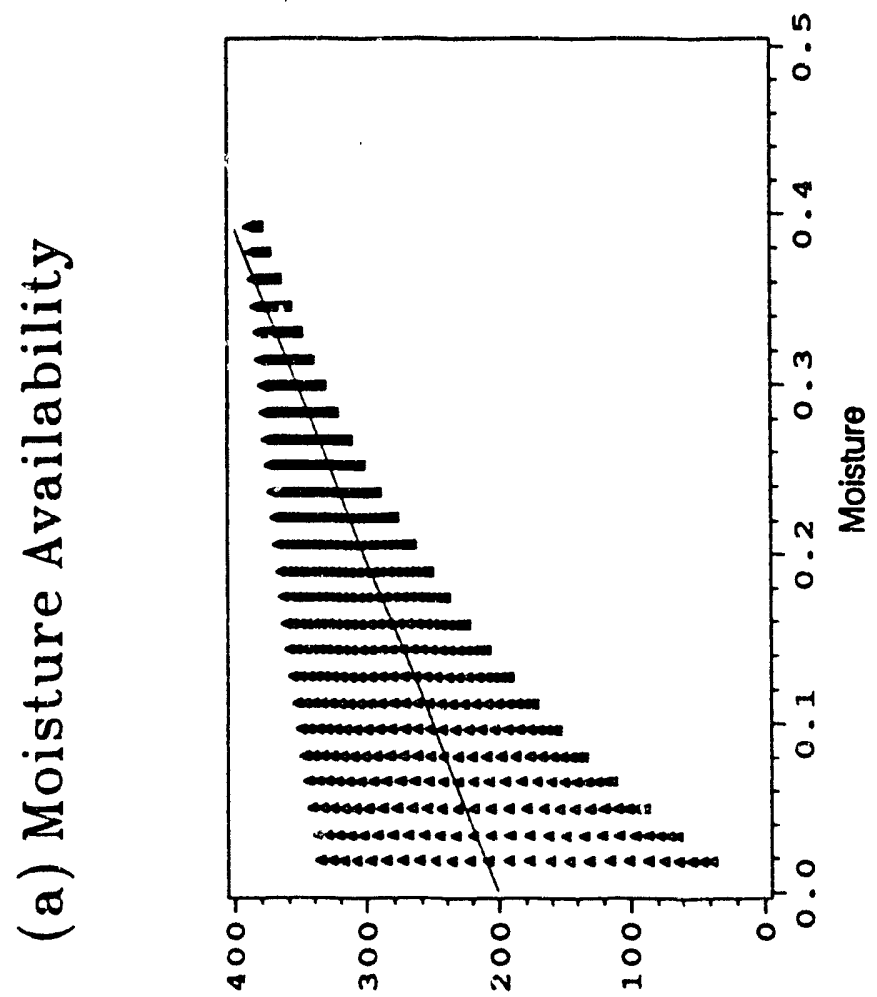

(w bs/\$HEW xn! wele) 

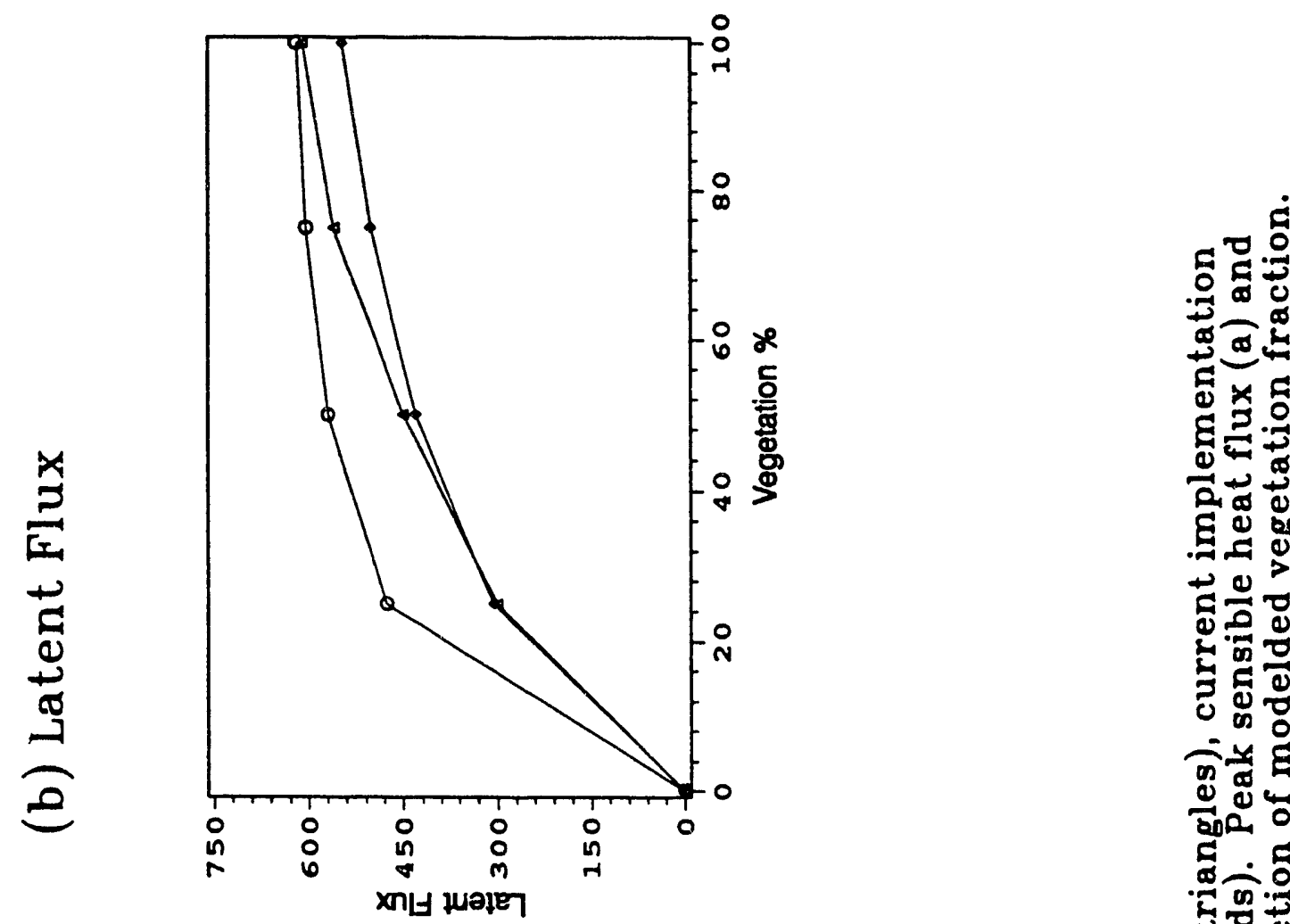

(1) . 움 임 음 0 界

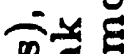
过 ‥ 도 ๘ं 동 $\pm 4$ ㅇ. 两 政 미 ๘ or 的 《U 능 c둥 昌占 की 용 द्व 0.7 记

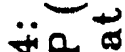

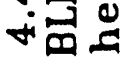
임 5 . 舟 


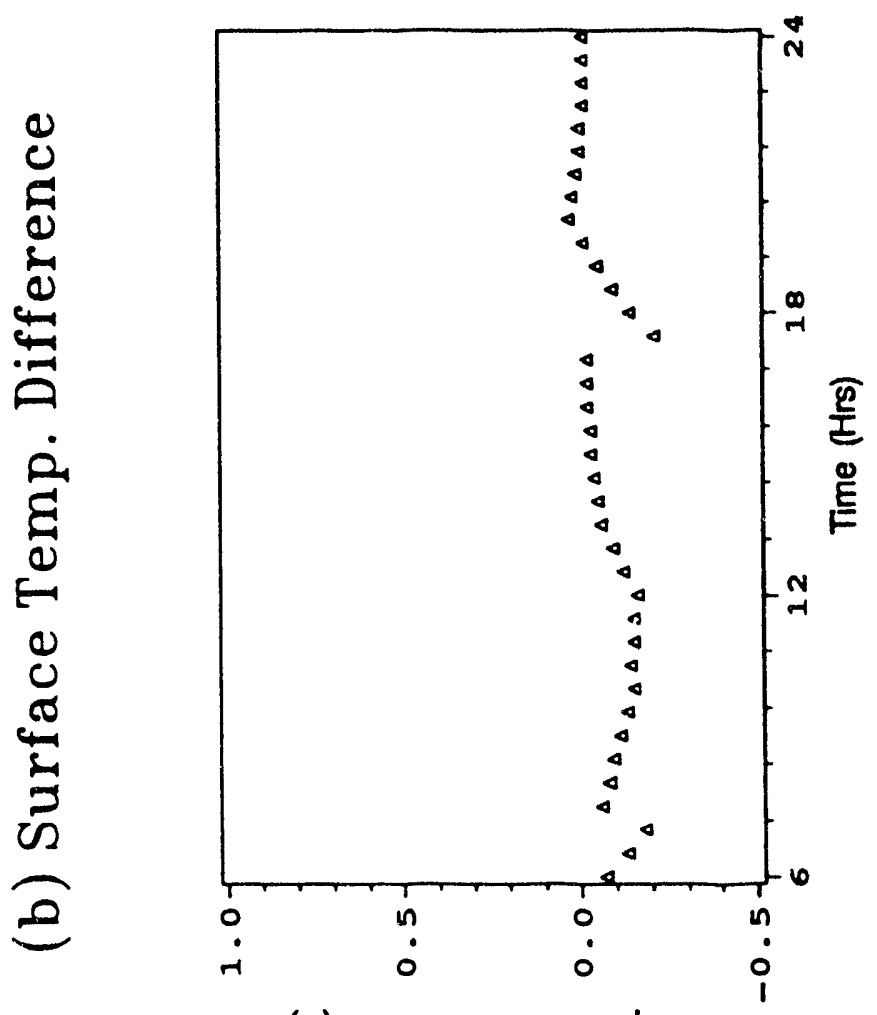

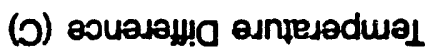
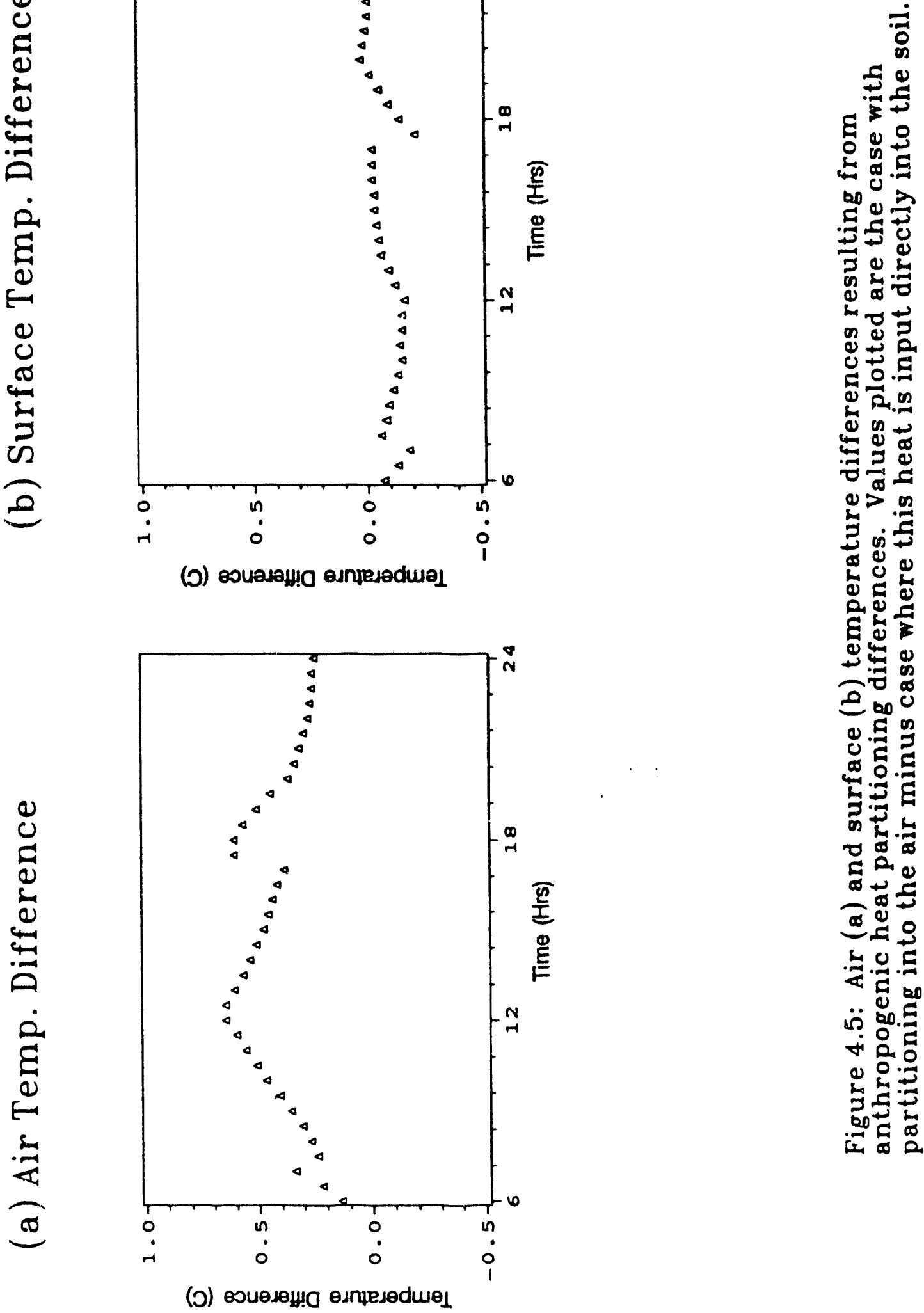


\section{CHAPTER 5 \\ Meteorological Modeling of the Los Angeles Basin}

\section{INTRODUCTION}

These three-dimensional meteorological simulations of the South Coast Air Basin (SoCAB) were developed to address several key issues in urban meteorology. One important aspect is the investigation of the importance of using detailed and realistic representations of the surface characteristics in the basin. Air quality scientists, who use mesoscale models to provide the meteorological inputs for photochemical models generally neglect the variability of surface characteristics within their modeling domains. The only previous study of the Los Angeles Basin to include a detailed surface characterization was that of Ulrickson (1992), which concluded that the impact of detailed surface characteristics on local wind fields and mixing heights was minimal. Ulrickson, neglected, however, to include a detailed analysis of the air temperature impacts which could be of great significance to air quality simulations and predictions of electric utility demand implications. Furthermore, accuracy of the surface characterizations used in that study was limited by the use of Land Use and Development Area (LUDA) data, which are heavily biased toward non-urban land-use categories.

Other notable numerical studies of the interaction between the urban environment and a sea-breeze climate include the works of Yoshikado (1990, 1992), and Yoshikado and Kondo (1989) which focused on the Tokyo bay. This present study expands upon Ulrickson's work by using an improved 
specification of surface land use. In addition, the necessary temperature analysis is conducted, resulting in a useful discussion of air quality and energy use implications associated with a heterogeneous surface.

The sensitivity study described in Chapter 3 was useful as a scoping tool, since it investigated the general impact of various surface characteristic modifications on local meteorological flow. Two-dimensional simulations are so computationally inexpensive that investigation of over twenty different scenarios was reasonable. Although these simulations produced useful and interesting results, one must question the applicability of such idealized simulations to the study of the actual meteorology in a three-dimensional domain. The twodimensional simulations are likely to over-estimate the impacts of surface variability since in three dimensions there is an increase in terrain-induced mixing, and an addition of a third direction for diffusion. Also, the two-dimensional study was idealized in that it specified minimal geostrophic wind speeds. In the presence of a realistic synoptic flow, the signature of surface variability will be diffused further. For these reasons, full three-dimensional simulations are necessary to develop a realistic understanding of surface influences.

The three-dimensional simulations were conducted in several parts. First, a homogeneous surface base case was developed to approximate the present state of meteorological modeling used by air quality scientists. This case makes use of the homogeneous surface assumption and domain-wide constant values of each surface characteristic. Improvements were then made to this case and a new heterogeneous case was developed using an improved land-use-based characterization of the urban surface. Anthropogenic heat release was then added to this case resulting in a final base case which represents a best estimate of the current state of the Los Angeles Basin. The next phase of meteorological simulation 
involved development and implementation of surface modification cases. The two types of modifications investigated are albedo and vegetation. These modifications are applied separately and also in combination, using two different modification levels; moderate and high. The results of these simulations are discussed in terms of their ability to cool the urban environment and reduce the production of photochemical smog. A discussion of the modified wind fields is also included to explain the impact on advection and diffusion of pollutants.

The computational domain for these simulations is illustrated in Figure 5.1a. The domain of interest for air quality modelers is the Los Angeles Basin and the San Fernando Valley. The Los Angeles basin is typically divided into a western and eastern portion, separated by the Puente hills and Santa Ana mountains. The basin is enclosed by the San Gabriel mountains to the north, the San Bernardino mountains to the north-east, the San Jacinto mountains to the east, and the ocean to the west. There are several major routes through which the sea breeze and synoptic-scale flow exits the basin; the Banning Pass in the east, and the Cajon and Newhall passes in the north.

The modeling domain spans $325 \mathrm{~km}$ in the east-west direction and $200 \mathrm{~km}$ in the north-south direction $(65,000 \mathrm{sq} . \mathrm{km})$. This domain is located in zone 11 of the standard Universal Transverse Mercator (UTM) geographical positioning system. The computational grid uses 22 variably spaced vertical levels extending from the surface to $9000 \mathrm{~m}$ ( the tropopause). While the horizontal domain extends well beyond the extent of the basin, it should be emphasized that results near the non-ocean boundaries are particularly unreliable due to boundary effects. Simulations have demonstrated that the southwestern boundary, composed of ocean-filled grid cells, is much less subject to edge effects. This is probably a result of the homogeneous, flat nature of these areas, and the fact that they 
contain ocean with a specified constant surface temperature boundary condition.

The geographic focus for analysis purposes is limited to the Los Angeles Basin and San Fernando Valley. For this reason, an analysis subdomain has been defined for presentation of simulation results. This subdomain, presented in Figure 5.1b, covers 15,000 sq. $\mathrm{km}$, extending from UTM coordinates $(350,3700)$ to $(500,3800)$ encompassing $23 \%$ of the entire computational domain. This region consists of the western basin containing Los Angeles and Anaheim; the eastern basin with Riverside and San Bernardino; and the San Fernando Valley containing Van Nuys and Burbank. The model results in this reduced-size domain are believed to be more robust as they are free from domain edge effects.

The geostrophic winds were taken from sounding data obtained from the Los Angeles airport at 4:00 a.m. on August 28, 1987. This particular sounding profile represents a fairly calm pre-dawn situation. It should be emphasized that synoptic flow patterns can greatly influence the impact of surface characteristics on mesoscale meteorology, with the surface signature being most prevalent under synoptically calm conditions. These are also the conditions which result in the most significant air quality problems and the highest energy demand. It is therefore appropriate to conduct these simulations subject to this mild, but realistic level of geostrophic forcing. The necessary initialization profiles of temperature and humidity were also taken from the August 28 data set. These initialization profiles, presented in Table 5.1, are representative of a typical pre-dawn stable situation in the L.A. basin with a thermal inversion at roughly $800 \mathrm{~m}$. The model requires specification of additional parameters including latitude and Julian Day (day of the year, 1-365). The latitude of the basin, used to calculate solar angles, is $34^{\circ}$. The day chosen for all of the simulations in this research was August 28, Julian Day 240. 
Table 5.1: Initial domain-wide sounding profiles

\begin{tabular}{|rcccc|}
\hline $\begin{array}{c}\text { Altitude } \\
(\mathrm{m})\end{array}$ & $\begin{array}{c}\text { Air } \\
\text { Temperature }(\mathrm{K})\end{array}$ & $\begin{array}{c}\text { Specific } \\
\text { Humidity }\end{array}$ & $\begin{array}{c}\text { Wind } \\
\text { Direction }\end{array}$ & $\begin{array}{c}\text { Wind } \\
\text { Speed }(\mathrm{m} / \mathrm{s})\end{array}$ \\
\hline 5. & 289.4 & .0130 & 352.0 & 2.00 \\
30. & 289.5 & .0130 & 352.0 & 2.12 \\
100. & 289.2 & .0130 & 337.0 & 1.30 \\
200. & 289.3 & .0126 & 0.0 & 0.50 \\
300. & 290.0 & .0118 & 45.0 & 0.57 \\
400. & 292.3 & .0108 & 128.0 & 1.14 \\
500. & 294.6 & .0092 & 90.0 & 2.60 \\
600. & 295.8 & .0082 & 75.0 & 3.42 \\
700. & 298.4 & .0074 & 49.0 & 3.47 \\
800. & 299.6 & .0060 & 63.0 & 3.94 \\
1000. & 299.2 & .0050 & 146.0 & 0.72 \\
1200. & 297.0 & .0050 & 58.0 & 0.94 \\
1500. & 293.3 & .0050 & 22.0 & 2.15 \\
2000. & 288.6 & .0048 & 85.0 & 2.51 \\
2600. & 283.1 & .0045 & 110.0 & 5.31 \\
3200. & 278.1 & .0030 & 92.0 & 7.00 \\
4000. & 273.1 & .0020 & 96.0 & 10.06 \\
5000. & 266.8 & .0015 & 111.0 & 4.40 \\
6000. & 260.6 & .0008 & 198.0 & 0.63 \\
7000. & 254.3 & .0005 & 241.0 & 3.54 \\
8000. & 248.1 & .0005 & 241.0 & 3.00 \\
9000. & 241.8 & .0005 & 241.0 & 3.00 \\
\hline
\end{tabular}

The analysis of the simulation results includes a number of data presentation techniques. The results for several important cases are analyzed in detail at specific simulation times: 1000, and 1500 Local Standard Time (LST). The results at 1000 LST are useful for understanding the formation of photochemical smog whereas the afternoon results are useful in terms of understanding the potential impacts of surface characteristics on peak energy demand. In addition, diurnal profiles of meteorological variables are analyzed at the grid cells corresponding to representative sites in the western basin, eastern basin, and San Fernando Valley. These three sites are Los Angeles (City Hall), Riverside (Parkview Hospital), 
and Burbank (airport). The exact location being considered is specified since each city actually overlaps several surface grid cells.

The city of Los Angeles is the major focus of this study. City Hall is located at UTM coordinates $(385.5,3769)$ near the intersection of Interstate 101 and the Harbor Freeway (11), and is roughly $20 \mathrm{~km}$ inland from the coast. Profiles of prognostic variables for Los Angeles are of particular interest since this is a location with a large population density, resulting in high energy demand and a significant air pollution problem. In addition, Los Angeles is relatively close to the coast, such that modifications in the sea breeze may result in significant meteorological impacts.

The city of Riverside, UTM coordinates $(459.5,3753)$, is located roughly 75 $\mathrm{km}$ to the east and $15 \mathrm{~km}$ south of Los Angeles, and over $50 \mathrm{~km}$ from the coast. Riverside is an important location, since it has historically been a location of very poor air quality. Unlike Los Angeles, Riverside is sufficiently far inland that the penetration of the sea breeze has only minimal impact on its local meteorology late in the day.

Burbank, located at UTM coordinates $(362.5,3786)$ is in the San Fernando Valley, approximately $25 \mathrm{~km}$ northwest of Los Angeles. This was chosen as the third analysis location since it is a heavily populated area located in the northwestern extreme of the SoCAB.

Diurnal profiles of the prognostic and diagnostic variables at these sites are useful in two ways. First, they demonstrate the development of the mesometeorology over the course of the day, identifying the times of day most affected by the various surface modifications. In addition, comparison of the diurnal profiles at different sites clarifies the role of geographical location (distance from the coast and position relative to mountains) in determination of the 
meteorological impacts of surface modifications. Finally, it is important to identify meteorological impacts in representative sites in the western basin, eastern basin, and San Fernando Valley, so that a realistic understanding of the relationship between urban surface characteristics and urban climate may be developed for highly populated regions.

\section{HOMOGENEOUS SURFACE CASE}

The homogeneous surface case was developed to represent the level at which air quality scientists currently model the meteorology in the SoCAB. This case is virtually the simplest level at which one can conduct a three-dimensional simulation. The only detailed surface data provided to the model are the landwater interface (coastline), and the topographical height of each grid cell. Throughout the land portion of the computational domain, all surface characteristics (albedo, roughness, moisture, and substrate properties) remain constant at representative default values. These values were based on data found in various sources (Hjelmfelt, 1982; and Oke, 1987), and are summarized in Table 5.2.

Table 5.2: Surface characteristics for the homogeneous surface case

\begin{tabular}{|ll||lc|}
\hline \multicolumn{2}{|c||}{ Surface Properties } & \multicolumn{2}{c|}{ Substrate Properties: } \\
\hline albedo & 0.16 & specific heat capacity $\left(\mathrm{g} \mathrm{g}^{-1} \mathrm{~K}^{-1}\right)$ & 1.34 \\
roughness length $(\mathrm{m})$ & 0.19 & density $\left(\mathrm{g} \mathrm{cm}^{-3}\right)$ & 1.94 \\
moisture availability & 0.11 & thermal diffusivity $\left(\mathrm{cm}^{2} \mathrm{~s}^{-1}\right)$ & 0.0036 \\
\hline
\end{tabular}




\subsection{Wind Speed Vectors}

Because of the relatively mild specified geostrophic forcing, the wind profiles observed in the simulation results are due primarily to influences from surface topography, surface characteristics, and the generation of a coastal seabreeze. In this particular case the homogeneous surface assumption is associated with domain-wide constant values for each surface characteristic. Thus, the generated circulations are entirely due to sea breeze and topographical effects.

The wind profile in the basin subdomain at 1000 LST is beginning to develop, as presented in the vector plot of Figure 5.2a. There is a peak velocity of $6.4 \mathrm{~m} \mathrm{~s}^{-1}$ blowing south over the San Gabriel mountains. It is suspected that the hydrostatic assumption, which is an integral part of the CSUMM model formulation, exaggerates mountain-valley flow patterns. Off the coast a moderately strong south-westerly flow is essentially perpendicular to the coastline. So, while the strongest circulations are associated with topographical effects, a sea breeze is clearly developing. Also of note is a mild recirculation zone in the wake of Santa Catalina island in the south-western corner of the analysis domain.

The coastal flow develops over the course of the day, resulting in a prominent sea breeze and well-developed mesoscale circulation, Figure 5.2b. In the late afternoon, the peak velocity in this domain has increased to $6.8 \mathrm{~m} \mathrm{~s}^{-1}$, and the strength of the flow throughout the basin has increased substantially. In contrast to results earlier in the day, the eastern basin is starting to experience much stronger surface-generated westerly winds. These winds are strongest in the eastern basin in the vicinity of Riverside and San Bernardino. The timing and extent of this sea-breeze circulation are important since the sea breeze plays an important role in the mixing and transport of pollutants. The sea breeze is also very important as it provides a source of cool ocean air for replenishing the air in 
the polluted basin.

\subsection{Air Temperature Contours}

Temperature contours for this homogeneous case are relatively parallel to the coastline and topographic features, with deviation from this shape increasing with distance inland. At 1000 LST the temperature contours, Figure 5.3a, indicate that the entire basin is relatively cool, below $25^{\circ} \mathrm{C}$. North and east of the basin the inland air is warmer with a temperature range of 25 to $30^{\circ} \mathrm{C}$. Later in the day, as illustrated in Figure 5.3b, temperatures over the entire basin are in the 25 to $30^{\circ} \mathrm{C}$ range. While Burbank, Los Angeles, and Anaheim are at roughly $26^{\circ} \mathrm{C}$, Claremont and Riverside, being further removed from the sea breeze are several degrees warmer. These figures are instructive as they identify the large temperature swings in the basin which result solely from sea breeze and topographical effects.

\subsection{Diurnal Temperature and PBL Height Profiles}

Diurnal temperature profiles at 3 locations in the basin are presented in Figure 5.4a. Los Angeles and Burbank are cooler than Riverside by roughly $2^{\circ} \mathrm{C}$ throughout the morning hours, and in the afternoon Los Angeles is cooler than Riverside and Burbank by almost 3 and $1.5^{\circ} \mathrm{C}$ respectively. The temperature of Los Angeles peaks earliest at about 1400 LST, and Riverside and Burbank both peak around 1500 or 1600 LST. Los Angeles is the coolest of the cities since it is nearest the coast and most affected by the sea breeze. Results presented later in this chapter indicate that surface characteristic variability produces temperature swings large enough to compete with the sea-breeze effect. 
Figure 5.4b presents the diurnal diagnosed planetary boundary layer (PBL) height for these cities. Since the PBL height is diagnosed based on levels of turbulent kinetic energy the result is a non-smooth function of time. For each of the three analysis cities the PBL height peaks in the mid-afternoon. The PBL height above Los Angeles is consistently 100 to $200 \mathrm{~m}$ higher than for Burbank or Riverside, probably due to the strong sea breeze over Los Angeles.

\subsection{Vertical Structure of Winds and Temperatures}

The vertical structure of the sea breeze flow through Los Angeles is illustrated in Figure 5.5. This figure is a side view of the basin with the ocean to the west (left) and downtown Los Angeles located at $x=385.5$. The lowest contour represents the surface topography which is greatly exaggerated due to the differing horizontal and vertical scales. The vertical velocity component is multiplied by a factor of 10 in this and all subsequent vertical cross-section plots for presentation purposes. In the morning the peak wind speeds of $4.0 \mathrm{~m} \mathrm{~s}^{-1}$ occur at elevations between 400 and $800 \mathrm{~m}$. In the afternoon, however, the peak winds are adjacent to the surface. This shift in the vertical location of the peak winds is likely due to the increased influence of the surface characteristics in the afternoon, as the surface heats up. The magnitude of the sea breeze has increased substantially in the afternoon to a peak value of $7.4 \mathrm{~m} \mathrm{~s}^{-1}$. A nominal transport speed of $5 \mathrm{~m} \mathrm{~s}^{-1}$ corresponds to $18 \mathrm{~km} \mathrm{~h}^{-1}$. Since the sea breeze is relatively small until about 1000 LST, Los Angeles should feel the effects of this flow by 1100 LST, compared with Riverside which is not exposed to the sea breeze until about three hours later. 


\section{UTZ HETEROGENEOUS SURFACE CASE}

The next level of sophistication in modeling the SoCAB is to use an approximation of the actual distribution of the various surface characteristics throughout the computational domain. This approximation has been made using an Urban Terrain Zone (UTZ) data base which specifies the distribution of land-use in each of the $5 \mathrm{~km}$ grid cells. As explained in Chapter 4, representative values of surface characteristics were assigned to each terrain type. In order to calculate an aggregated value of a given surface characteristic in a grid cell, the areaweighted average of the terrain-type values was used. Table 5.3 summarizes the ranges and averages of the characteristic values used in this heterogeneous surface case.

Table 5.3: Surface characteristics for the heterogeneous surface case

\begin{tabular}{|c|c|c|c|}
\hline Parameter & Average & High & Low \\
\hline \multicolumn{4}{|c|}{ Surface Properties } \\
\hline albedo & 0.16 & 0.20 & 0.14 \\
\hline roughness & 0.17 & 1.00 & 0.05 \\
\hline moisture & 0.10 & 0.24 & 0.01 \\
\hline \multicolumn{4}{|c|}{ Substrate Properties: } \\
\hline specific heat capacity $\left(\mathrm{Jg}^{-1} \mathrm{~K}^{-1}\right)$ & 1.34 & 1.76 & 1.00 \\
\hline density $\left(\mathrm{g} \mathrm{cm}^{-3}\right)$ & 1.90 & 2.29 & 1.52 \\
\hline thermal diffusivity $\left(\mathrm{cm}^{2} \mathrm{~s}^{-1}\right)$ & 0.0040 & 0.0057 & 0.0026 \\
\hline
\end{tabular}

During initial simulations, the default values used in the homogeneous surface case were tuned so that they closely matched the average values of this heterogeneous case. Thus, the two cases can be compared, revealing the meteorological impacts of the homogeneous surface assumption. 
A similar listing of surface and substrate properties is given in Table 5.4 for the values at the specific grid cells corresponding to the analysis cities of Riverside, Burbarik, and Los Angeles.

Table 5.4: Surface characteristics for specific cities

\begin{tabular}{|l|cccc|}
\hline Parameter & Domain Avg. & Riverside & Burbank & Los Angeles \\
\hline \hline \multicolumn{5}{c|}{ Surface Properties } \\
\hline albedo & 0.160 & 0.168 & 0.152 & 0.153 \\
roughness & 0.170 & 0.304 & 0.339 & 0.446 \\
moistus & 0.100 & 0.102 & 0.138 & 0.053 \\
\hline \hline \multicolumn{5}{|c|}{ Substrate Properties: } \\
\hline specific heat capacity $\left(\mathrm{g} \mathrm{g}^{-1} \mathrm{~K}^{-1}\right)$ & 1.34 & 1.30 & 1.34 & 1.13 \\
density $\left(\mathrm{g} \mathrm{cm}^{-3}\right)$ & 1.90 & 1.73 & 1.99 & 1.63 \\
thermal diffusivity $\left(\mathrm{cm}^{2} \mathrm{~s}^{-1}\right)$ & 0.0040 & 0.0038 & 0.0031 & 0.0039 \\
\hline
\end{tabular}

The albedo for each of these cities is very close to the domain-wide average value of 0.16 . As would be expected, the roughness of Los Angeles is the highest of these cities and all three urban locations have roughness values substantially above the domain average. Also of no great surprise is the fact that Burbank and Riverside have higher moisture availability than Los Angeles. The substrate properties, however, do not vary significantly enough among these locations to be of much consequence.

\subsection{Wind Speed and Temperature Differences}

Figure 5.6a illustrates the subdomain horizontal flow field for the base case simulation at 1500 LST. This figure is a vector difference between the heterogeneous and homogeneous case winds. The peak velocity difference is $2.9 \mathrm{~m} \mathrm{~s}^{-1}$. The general character of the flow fields are similar, with several notable differences: the coastal breeze appears to be enhanced slightly in this heterogeneous 
case; flow in the western basin is shifted north; and flow in the southern portion of the eastern basin is deflected south by a significant amount.

Evidence of an urban "heat island" would be difficult to isolate because of the sprawling nature of Los Angeles. Figure 5.6b, however, provides some evidence of this effect. This plot is the 1500 LST temperature difference between the heterogeneous and homogeneous cases. Each solid contour line represents a region where the heterogeneous case is $0.5^{\circ} \mathrm{C}$ warmer than the homogeneous case. The dashed lines represent regions where the heterogeneous case is cooler. The homogeneous surface assumption thus results in an underestimation of urban air temperatures in excess of $1^{\circ} \mathrm{C}$ throughout the western basin. This homogeneous assumption therefore misses the heat island effect which results from surface characteristic variability.

\subsection{Diurnal Temperatures and PBL Heights}

The diurnal profiles of air temperature and PBL height are shown in Figure 5.7 for the three analysis cities. In the morning, Los Angeles is consistently warmer by roughly $1^{\circ} \mathrm{C}$, but by 1400 LST both Burbank and Riverside are warmer than Los Angeles. This result is in contrast to Figure 5.4a which indicated that Riverside was warmest throughout most of the day for the homogeneous case. The change in temperature profiles can be traced to changes in surface characteristics in the heterogeneous case. In this case the albedo and moisture availability of Los Angeles are significantly lower than for Riverside (see Table 5.4). The surface characteristic impact on PBL height is illustrated in Figure 5.7b. While the PBL height for Riverside and Burbank have remained relatively unchanged from the homogeneous case, the PBL height over Los Angeles has increased by about $100 \mathrm{~m}$. This has important consequences in predictions of 
photochemical smog concentrations. Because the PBL height is directly related to pollutant concentrations, a higher PBL will result in lower concentrations of photochemical precursors and a concomitant lowering predicted smog levels. To the first order the error in L.A. smog predictions resulting from the homogeneous surface assumption would be $(100 \mathrm{~m}) /(600 \mathrm{~m})$ or about $15 \%$. This estimate uses the simplifying assumptions that precursor concentrations are proportional to the volume of the mixed layer, and that photochemical smog levels are proportional to precursor concentrations.

\section{ANTHROPOGENIC HEATING CASE}

The previous cases ignore the presence of anthropogenic heating in the highly urbanized Los Angeles Basin. Since two-dimensional simulations have indicated that anthropogenic heating may result in noticeably elevated air temperatures, this simulation case was developed to investigate the implications of anthropogenic heat release in a three-dimensional domain. This case is identical to the heterogeneous base case, except that an anthropogenic heat source term has been added to the energy balance of the near-surface air. The profile shape and magnitude are given in Chapter 4. This case is the most accurate representation of the current physical characteristics of Los Angeles, and is used as the departure point for the mitigation scenarios of the following section. All subsequent comparisons will refer back to this case as the base case.

Comparing wind profiles and mixing heights between this base case and the previous heterogeneous case, no significant differences were found, and so a detailed discussion of these profiles is not warranted. Figure 5.8a shows the wind vector difference at 1500 LST between the heterogeneous case with anthropogenic heating and without anthropogenic heating. The impact on wind speeds 
is fairly small, not exceeding $0.8 \mathrm{~m} \mathrm{~s}^{-1}$. The air temperature differences associated with this base case were also quite small. Figure $5.8 b$ is the corresponding temperature difference plot. The most significant temperature impacts of $0.25^{\circ} \mathrm{C}$ occur east of Los Angeles and in the San Fernando valley. The sea breeze is responsible for advecting this air temperature impact inland. Thus, earlier in the day, the peak effect was found to be centered further west over the city of Los Angeles. While inclusion of anthropogenic heating has a minimal effect on the meteorology, it is worth including since it improves our ability to model this domain and identify the heat island. In addition, a $0.25^{\circ} \mathrm{C}$ change in air temperature is significant when compared with the other temperature impacts in this study. Furthermore, future extensions of this work may include wintertime simulations with elevated levels of anthropogenic heating. Under such conditions, the inclusion of the anthropogenic heating term may become quite important.

\section{MITIGATION SCENARIO DEVELOPMENT}

An important driving force for this research was the interest in determining the potential for active mitigation of urban pollution and heat island problems through implementation of programs and policies which seek to modify the existing urban surface in a positive way. Two examples of mitigation schemes are urban tree planting and urban lightening (high albedo) programs. The following sections describe the development of some prototypical mitigation schemes, and the resulting meteorological modifications and air quality implications based on three-dimensional simulations. Evaluation of these mitigation schemes will completely bypass the issue of implementation, which is left for other researchers to investigate. 
Developing mitigation scenarios for these simulations consisted of three steps. First was the identification of grid cells in the computational domain which could be realistically modified. It was then determined which of these cells had the need or potential for modification. Finally, the extent to which each cell could be modified was determined.

The domain consists of 65 grids in the east-west direction and 40 grids in the north-south direction, corresponding to 2600 surface grid cells. It would be inappropriate to assume that a mitigation scheme would be able to alter all of these grid cells, many of which are covered with ocean, desert, or forest. Therefore, the following method was used for filtering these 2600 grid cells to obtain two smaller sets of grid cells. One set was designated for vegetative modification, and the other was subject to albedo modifications. It is important to note that these two sets could be identical or may simply overlap, depending upon the rules applied to the filtering of grid cells for each mitigation strategy. The potential for albedo modification is highest in the urban core and high-density residential areas where artificial surfaces may be readily painted or resurfaced with lighter colors. The potential for vegetation modification, on the other hand is largest in sparsely built residential and commercial areas where large regions of bare soil may be used for tree planting programs. In both cases, natural areas are unlikely candidates for modification.

Natural areas were defined as grid cells which contain less than $20 \%$ urbanized land use. This was accomplished using the information in the gridded urban terrain zone data base as discussed previously. These grid cells were then eliminated from consideration. Thus, desert, ocean, forest, and other natural areas were not considered for either albedo or vegetation modification. The result was 394 grid cells which were left to be considered for albedo and/or 
vegetation modifications.

It was then determined which of the remaining grid cells would benefit from albedo and tree planting schemes. The current albedo of each grid cell was calculated using the urban terrain zone data base and existing data relating each terrain zone to a typical albedo value. A threshold albedo was determined above which, a grid cell would not be modified. Specifically, if a grid cell had a preexisting albedo above 0.20 it was not considered for albedo modification. This restriction was imposed because it is unlikely that such a program (with its associated costs) would be implemented in a region where the potential improvement is not large. Since no land-use is assigned an albedo above 0.20 , however, there were no cells which were excluded based on this criterion.

The approach used in developing vegetation mitigation cases was slightly different. Rather than using the rough estimate of the existing vegetative cover of each grid cell, it was assumed that cells which contained residential or commercially-developed land would be candidates for varying levels of tree planting. Thus, each of the 394 cells would be assigned a given value of vegetation augmentation which would depend upon the makeup of each cell.

The final step in developing mitigation schemes was to determine the extent to which the remaining grid cells could be modified. For albedo, this issue was addressed by Martien et al. (1989) and Bretz et al. (1992) in an attempt to evaluate the potential for increasing the albedo of Sacramento, California. Data presented by Bretz show that the urban fabric of Sacramento consists of $28 \%$ rooftop, $16 \%$ street, $14 \%$ other impervious surfaces (including parking lots), and $42 \%$ vegetated and bare soil areas. With this makeup and existing high-albedo roof coatings and paving materials Martien postulates that the albedo of Sacramento could be increased by 0.15 . While Los Angeles and Sacramento have distinct 
differences, it is likely that L.A. consists of a higher percentage of impervious paved surfaces, and a higher density of housing, suggesting that the potential albedo modification for L.A. may actually be much higher than 0.15 . The first albedo modification scheme which was implemented in simulations used a conservative approach. It was assumed that each grid cell could have its albedo increased by up to 0.15 multiplied by the fraction of that cell which was composed of developed land. The resulting geographical distribution of albedo modification is concentrated in and around downtown Los Angeles as depicted in Figure 5.9a. Note that of the 2600 grid cells in the domain, only 394 have been marked for albedo modification. Since these cells are concentrated in downtown Los Angeles and along the coast, their impact on the basin's meteorology should be significant. Although the mean of the albedo modification is 0.082 , the majority of the modified cells had their albedo modified by less than 0.05 or more than 0.10 . The average modification, considering all 2600 surface cells of the domain is only 0.012 .

In order to assign appropriate levels of vegetative cover augmentation to each grid cell a baseline level representing a reasonable tree-planting program was needed. While it would be incorrect to assume that there is the same distribution of surfaces in Los Angeles and Sacramento, this is a good point of departure. While Sacramento contains $42 \%$ permeable surfaces one might expect Los Angeles to have less; assume $30 \%$. Furthermore, if one assumes that roughly half of this urban land is already covered with vegetation, or not conducive to vegetation, $15 \%$ of the developed Los Angeles Basin should be amenable to tree planting. Clearly, however, certain land-use types are more accommodating to tree planting than others. This baseline value is therefore attenuated by $20 \%$ for close-packed housing, the city core, and administrative land uses. Furthermore, 
this number is attenuated by $50 \%$ for the desert suburban land use due to the difficulty in caring for vegetation in such a dry region. So in general, developed land in the basin can be modified by increasing the vegetative fraction by a nominal 0.15 . Using this strategy, the average vegetation fraction increase for the 394 modified cells is 0.07 . The average modification, considering all 2600 cells of the domain, however, is only 0.01 . The distribution of the vegetation modification is shown in Figure 5.9b. Note that the distributions of albedo and vegetation modification are very similar, resulting from their similar land-use-based development.

The above approach was used to develop a series of albedo and vegetation mitigation schemes for simulation. Specifically, "moderate", "extreme", "combined moderate", and "combined extreme" scenarios were developed. In the "moderate" level of modification albedo or vegetation fraction are both increased by up to 0.15 within a single grid cell. In the "extreme" level, the modification is doubled to 0.30 . Results for the moderate, extreme, and combined scenarios are presented in the following section. Table 5.5 summarizes the albedo and vegetation modifications made in these scenarios. 
Table 5.5: Albedo and vegetation values before and after modification

\begin{tabular}{|l|cccc|}
\hline Case & Domain Avg. & Riverside & Burbank & Los Angeles \\
\hline \hline \multicolumn{5}{|c|}{ ALBEDO } \\
\hline Heterogeneous Base Case & 0.160 & 0.168 & 0.152 & 0.153 \\
Moderate Modification & 0.176 & 0.269 & 0.196 & 0.291 \\
Extreme Modification & 0.192 & 0.370 & 0.240 & 0.429 \\
\multicolumn{5}{|c|}{} \\
\hline \hline \multicolumn{7}{|c|}{ VEGETATION } \\
\hline Heterogeneous Base Case & 0.158 & 0.192 & 0.170 & 0.065 \\
Moderate Modification & 0.172 & 0.279 & 0.208 & 0.189 \\
Extreme Modification & 0.186 & 0.366 & 0.246 & 0.313 \\
\hline
\end{tabular}

\section{MODERATE LEVEL MODIFICATION RESULTS}

\subsection{Wind Profile Differences}

Application of either the moderate albedo or vegetation scenarios resulted in notable wind speed impacts. Figure 5.10 illustrates the wind vector differences between the (a) moderate albedo and (b) moderate vegetation cases relative to the base case simulation at 1000 LST. At the coast the sea breeze is weakened substantially in the albedo case and less so in the vegetation case. The wind speed impact in both cases is primarily along the Long Beach coast. The sea breeze flow around Claremont, UTM coordinates (434.5,3773), is actually strengthened. The magnitude of the peak impact is 0.47 and $0.37 \mathrm{~m} \mathrm{~s}^{-1}$ respectively for the albedo and vegetation cases. Noting that the peak 1000 LST wind speed for the base case was $5.4 \mathrm{~m} \mathrm{~s}^{-1}$, these values correspond to an approximate reduction in the sea breeze by 9 and $7 \%$. The strengthening of the sea breeze in the Claremont area and weakening of the sea breeze near the coast may correspond to the effect that cooling Los Angeles has on buoyant flow, the cooled air is denser resulting in a down-flow (or reduced up-flow) over the city. To 
conserve mass, air exits this region radially. This is evident in this figure, where the flow is superimposed over the baseline westerly sea breeze.

Figure 5.11 is the corresponding plot for 1500 LST. By this time in the afternoon, the effect of these moderate level modification schemes is quite significant throughout the analysis domain. The general result is a slowing of the sea breeze, depicted by difference vectors which point to the south-west. The peak wind speed at this time in the base case was $7.3 \mathrm{~m} \mathrm{~s}^{-1}$. The moderate albedo modification slows the sea breeze by up to $1.23 \mathrm{~m} \mathrm{~s}^{-1}$, or nominally by $17 \%$. The moderate vegetation modification has a less significant impact of $0.87 \mathrm{~m} \mathrm{~s}^{-1}$, or a nominal reduction of $12 \%$.

\subsection{PBL Height Differences}

The diurnal profile of PBL height reductions produced by these modifications has a noisy signal. General trends, however, are evident as shown in Figure 5.12. This figure displays the PBL height depression for Riverside, Los Angeles, and Burbank for both the (a) albedo and (b) vegetation cases relative to the base case. The albedo case results in PBL height depressions on the order of $50 \mathrm{~m}$, while the vegetation case impact is on the order of $25 \mathrm{~m}$. With the exception of Riverside at 0900 LST under the albedo modification, the PBL height is consistently lowered by the application of either of these modification scenarios. This is due to the fact that the cooler surface resulting from these scenarios produces less vertical motion in the atmosphere. Thus, the boundary layer height is reduced. As discussed previously, this reduction in the PBL height may provide a mechanism for increased levels of smog and other pollutants since the effective volume for mixing of surface emissions is reduced. 


\subsection{Temperature Differences}

The air temperature effects resulting from application of these mitigation strategies are of particular importance since they are related to both the issues of energy use and air quality. It was shown in Chapter 1 that reducing air temperatures in the Los Angeles Basin by $1^{\circ} \mathrm{C}$ for one hour is equivalent to more than $\$ 50,000$ in saved energy. This number should be considered as the following temperature results are presented. Figure 5.13 presents air temperature differences between the moderate modification cases and the base case at 1000 LST. Each contour represents a $0.25^{\circ} \mathrm{C}$ difference, indicating that the albedo modification is responsible for more than $0.25^{\circ} \mathrm{C}$ cooling throughout the basin, with a peak impact of $1^{\circ} \mathrm{C}$ in a small $\left(-75 \mathrm{~km}^{2}\right)$ region of eastern Los Angeles. The corresponding impact for the vegetation case is shown to be much smaller, exceeding $0.25^{\circ} \mathrm{C}$ primarily in the western basin and San Fernando valley.

Figure 5.14 presents the same data for 1500 LST. Here the contour levels are $0.5^{\circ} \mathrm{C}$. At this afternoon hour, the temperature impacts are much more significant. The albedo case cools most of the basin by more than $1^{\circ} \mathrm{C}$ and a significant area including L.A., Burbank, and Claremont is cooled by nearly $1.5^{\circ} \mathrm{C}$. Again, the vegetation case produces less significant results. The peak vegetation impact of nearly $1.5^{\circ} \mathrm{C}$ occurs in eastern Los Angeles and the northwestern San Fernando valley.

The diurnal temperature differences between these cases and the base case reveal an interesting effect. Figure 5.15 presents these profiles for Riverside, Los Angeles, and Burbank. The albedo plot, (a), indicates that the peak temperature effect of $1.6^{\circ} \mathrm{C}$ occurs in Los Angeles around noon. The peak impact for Riverside and Burbank, however, is delayed until about 1500 LST. In the vegetation case of (b), the peak effect in Los Angeles of $1.4^{\circ} \mathrm{C}$ also precedes the peak effect in 
Riverside and Burbank. The temperature in Riverside peaks latest in the day in both cases. Since Los Angeles is nearest the coast it is the first of these cities to experience the sea breeze, which has the tendency of smoothing out any surfaceinduced meteorological effects. Thus, since Riverside is furthest inland, the impact of the surface modifications remains strong later in the day.

\subsection{Vertical Structure Differences}

It is important to analyze the vertical structure of the meteorological impacts so that a true assessment of the potential air quality impacts can be made. In terms of energy use, the near-surface air temperature is most important. Photochemical reactions, on the other hand, take place throughout the vertical extent of the lower troposphere. Thus, the vertical depth of temperature modifications is as important as their magnitude. Figure 5.16 presents the vertical cross-section of the velocity vector differences and temperature differences between the moderate modification cases and the base case at 1500 LST. This cross-section cuts through downtown Los Angeles which is located at $x=385.5$. The lowest contour is the topography, and each additional contour represents a $0.5^{\circ} \mathrm{C}$ air temperature difference. The solid contours represent regions where the mitigation cases cool the air relative to the base case. The dashed contours are regions where the mitigation cases have actually warmed the air. This figure indicates that the air near the surface is cooled whereas a small volume of air at higher elevations is actually warmed. This dichotomy can be explained by two competing mechanisms. Both modifications result in a reduced heating load on the air near the surface. This effect is transmitted vertically through convective and diffusive transport. This is the mechanism responsible for cooling the air near the surface. But, because the surface is cooled, the driving force for the generated 
sea breeze is reduced and wind speeds are slowed by up to 2.8 and $2.2 \mathrm{~m} \mathrm{~s}^{-1}$ for the albedo and vegetation case respectively. The afternoon wind speed reduction is largest at elevations in the 400 to $800 \mathrm{~m}$ range. Near the surface this wind speed reduction is also significant but only about a quarter as large. This is the warming mechanism since the reduction in the sea breeze results in generally warmer air over land as less of the cool ocean air is allowed to ventilate the basin. Near the surface the energy balance effect dominates, and aloft the sea breeze effect dominates. Since the volume of air which is warmed is much smaller than the volume which is cooled, the net temperature effect on the rate of photochemical smog formation should still result in reduced concentrations. In addition, since the temperature-sensitive emissions occur at the surface, there is little question that in the summer these mitigation strategies will result in reduced emissions.

\section{EXTREME LEVEL MODIFICATION RESULTS}

The extreme level modifications produced meteorological impacts with the same characteristics as the moderate level cases. Although climate processes are governed by non-linear equations, the meteorological impacts from these simulations scaled in an approximately linear fashion between the moderate and extreme cases. Since the extreme level modifications were exactly double the moderate level, the resulting meteorological impacts were a factor of two more intense in the extreme modification cases. 


\subsection{PBL Height Differences}

Figure 5.17 presents the PBL height differences between the extreme modification cases and the base case for the three analysis cities. The albedo case resulted in PBL height depressions on the order of $100 \mathrm{~m}$ for all 3 cities. In the vegetation case the PBL was depressed by as much as $90 \mathrm{~m}$ over Los Angeles at 1300 LST. Riverside and Burbank, however, experience peak PBL depressions on the order of $50 \mathrm{~m}$.

\subsection{Temperature Differences}

Figure 5.18 presents the air temperature depressions resulting from the extreme modification cases. As was the case with the moderate modifications, Los Angeles experiences the largest temperature depression of 3.4 and $2.2^{\circ} \mathrm{C}$ for the albedo and vegetation scenarios respectively. These temperature impacts are roughly twice those found in the moderate modification cases, indicating that the temperature impact of increasing albedo or vegetation is also approximately a linear function of the modification level.

\section{COMBINED MODIFICATION RESULTS}

Figure 5.19 presents the air temperature depressions resulting from the (a) combined moderate and (b) combined extreme modification cases. The temperature impact of these combined schemes is actually larger than the addition of the individual impacts. In the combined moderate case, for example, Los Angeles is cooled by $3.6^{\circ} \mathrm{C}$ compared with 1.6 and $1.4^{\circ} \mathrm{C}$ for the individually applied moderate albedo and vegetation cases. If the impacts were simply additive, one would expect a combined moderate case temperature depression of only $3.0^{\circ} \mathrm{C}$. The actual depression was larger than expected by $20 \%$. Similarly, the combined 
extreme case resulted in a temperature reduction of $6.0^{\circ} \mathrm{C}$ compared with 3.4 and $2.2^{\circ} \mathrm{C}$ for the individually applied extreme albedo and vegetation cases. Thus, the combined application of vegetation and albedo modification strategies appears to have a slightly synergistic effect in terms of air temperature. It should be emphasized that this apparent effect is likely the result of the city of Los Angeles benefiting, not only from local modifications, but from modifications in nearby communities.

\section{SUMMARY}

The simulations presented here have provided insight into the relationship between urban surface characteristics and local meteorology. Both the accuracy of surface characteristic specification and the potential impact of surface modifications have been evaluated and shown to be significant.

The homogeneous surface characteristic assumption was applied in one simulation, and a much improved heterogeneous surface case was developed in another simulation. In comparing these two cases, it was found that the surface winds were only slightly modified, and air temperature deviations of slightly more than $1^{\circ} \mathrm{C}$ were found. The addition of mild profiles of anthropogenic heating was only found to increase air temperatures by roughly $0.25^{\circ} \mathrm{C}$, with relatively little impact on wind speeds.

Six surface modification schemes were investigated. These included the individual moderate and extreme augmentation of albedo and vegetative cover, and several combined modification cases. The moderate level cases were developed to represent plausible modifications which could be implemented fairly easily using available technology. The extreme cases are believed to represent the upper limits of plausibility, but should become more reasonable in 
the near future as more research effort is devoted to heat island mitigation. Increasing the albedo or vegetation fraction in the domain had similar general effects: the sea breeze was reduced; low level air temperatures were decreased; and the boundary-layer height was depressed. The magnitude of peak temperature depressions in Los Angeles for the moderate albedo and vegetation cases were around $1.5^{\circ} \mathrm{C}$. The corresponding P $\mathrm{LL}$ height depressions were 25 to $50 \mathrm{~m}$. The effects of the extreme modification cases were about double, and the combined cases showed an approximately additive effect. As was pointed out in the text, the depressed PBL height is a mechanism for increasing photochemical smog concentrations, and the reduced air temperature is a mechanism for improving air quality. The net tradeoffs between these two mechanisms must be thoroughly understood prior to recommending these mitigation strategies to policy-makers. This will require detailed photochemical simulations for the same computational domain. In the end, the conclusions of such paired meteorological and photochemical simuiations may be application specific, and thus preliminary results should be interpreted with caution.

\section{CONCLUSIONS AND FUTURE DIRECTIONS}

This dissertation has focussed on developing better simulation techniques for the highly heterogeneous surfaces associated with urban climates. Improvements to basic modeling strategies have been proposed and investigated. In addition several strategies for mitigating the urban heat island have been developed and investigated. The findings reveal that more work is necessary so that results of urban climate simulations can be interpreted with greater confidence, and used to shape public policy and future research. 


\subsection{Urban Modeling}

It has been shown that the specification of the urban surface characteristics for use in mesoscale models is a difficult and important aspect of simulating the urban climate. Two methods for obtaining subgrid-scale surface characteristics have been introduced. The method employed in this research has used a landuse data base and suppositions regarding the relationship between surface characteristics and land use categories. It has been suggested that use of satellite-derived remotely-sensed surface data would be a significant improvement in the specification of surface albedo, moisture availability, and vegetative cover. Since albedo and moisture have been identified as the most significant surface characteristics in terms of the ability to affect local climate, the incorporation of satellite data in future studies may be the single most important improvement to the modeling of urban climate. While the issues of calibration of these data and accounting for atmospheric attenuation need to be studied further, satellite data are already making great improvements in the ability to model large heterogeneous domains.

It must be emphasized that all simulations in this work have used surface grid cells which were $5 \mathrm{~km}$ in each horizontal dimension and $25 \mathrm{~m}$ in the vertical dimension. Thus, the temperature reported for downtown Los Angeles really represents a model prediction for a $0.125 \mathrm{~km}^{3}$ volume of air. The temperature reported for this volume of air is then an average value, and the actual temperature at locations within the corresponding grid cell may span a range of $5^{\circ} \mathrm{C}$ or more. Because of the limitations of the model, the details of the urban canopy are not considered in the simulations. Thus, the low-level air temperature predictions must be considered to be only first order estimates. A future improvement to this modeling strategy would be to include the urban canopy effects through 
the combined use of a canopy layer model and a mesoscale model as outlined in Chapter 2. Ultimately, however, monitoring studies must be conducted to provide a strong base of measured data for validation of model results. In addition to these monitoring studies, wind-tunnel measurements may provide insight to the relationship between the flow within the urban canopy and the overlying mesoscale flow which has been predicted in this research.

Another important drawback of this research is that it has focussed on a typical summer day in the Los Angeles Basin. The initialization profiles taken from the L.A. International Airport were in fact assumed to be representative of the entire computational domain. The corresponding error is believed to diminish as the simulation proceeds away from the initialization time of 0400 LST. For this reason, results were only presented for times after 0600 LST, and the focus was on times much later in the day. Future research should target other geographical areas, different synoptic weather patterns, and several times of the year.

\subsection{Mitigation Strategies}

The mitigation strategies developed and investigated in this chapter represent a very simplified approach. Future studies need to be developed to delve into many unresolved issues.

The implementation of a high albedo program is complicated in several ways. First, it must be decided which level the program is to be guided at. Should the state, county, or municipality take the lead on high-albedo legislation? Or, should individual utility companies or air quality districts be charged with developing incentive plans? Once the legislation or incentive plans are in place, various coatings and building materials need to be evaluated with respect to their albedo and other thermal properties. In the end there are issues of cost 
and benefit as well as aesthetics. For example, it is possible that high-reflectivity road surfaces and single-story rooftops will result in undesirable glare. While many Mediterranean "white-washed" cities do not appear to have significant complaints regarding aesthetics, it would be difficult to gauge the possible reaction of the American people. All of these issues need to be thoroughly evaluated or tested before major programs are implemented.

There are similar issues to be considered with regard to vegetation augmentation strategies. Identifying a group of suitable tree families for a particular climate is a significant problem in of itself. Trees planted in the Los Angeles Basin, for instance, should be drought-resistant, and low emitters of monoterpene and isoprenes. Trees also require a certain amount of care, especially in the first few years. Root systems can be responsible for damaging sidewalks and streets, and tall branches can interfere with overhead power lines. These and other issues all play a role in determining the usefulness of massive tree-planting programs.

\subsection{Initial Indications and Future Research}

The work presented here has clearly indicated that, in terms of airtemperatures and urban energy use, the investigated mitigation strategies are quite promising. The results in terms of air quality have been complicated by the potentially negative effects of lowering the boundary-layer heights and slowing of the sea breeze. Clearly, more work is required to evaluate the air quality trade-offs which have been identified. The first step is to use the meteorological output as input to a photochemical model. The resulting simulations will provide a quantitative evaluation of the impact of surface modifications on air quality. 

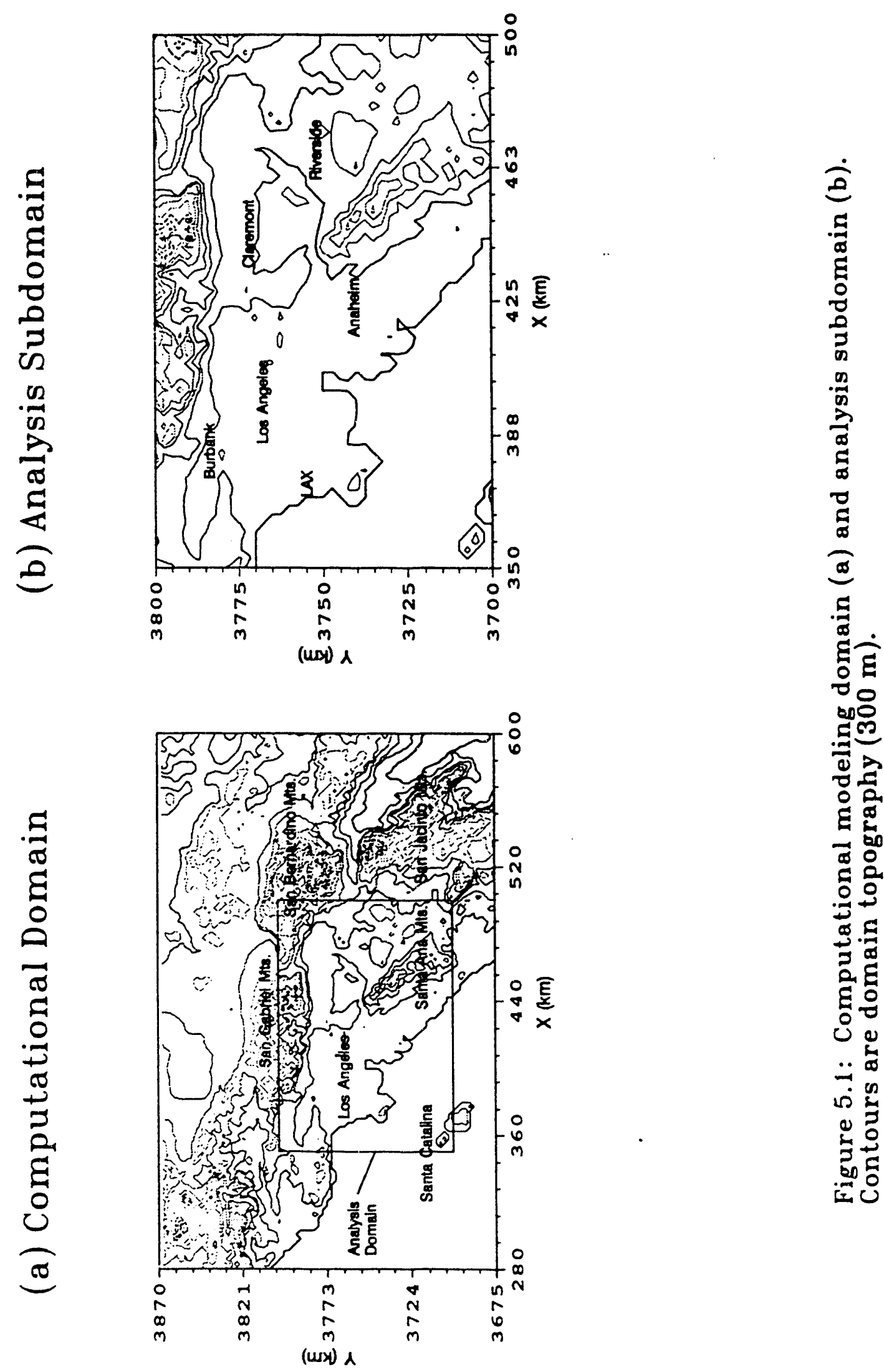


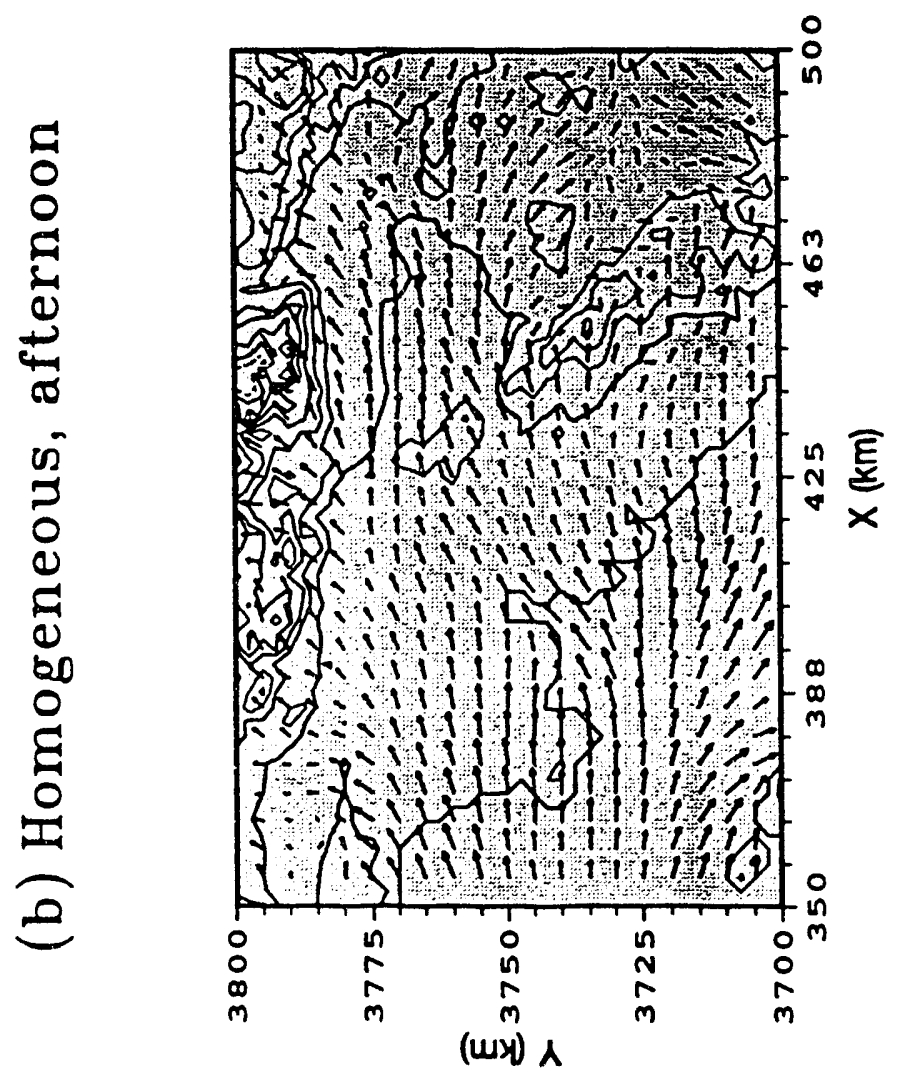

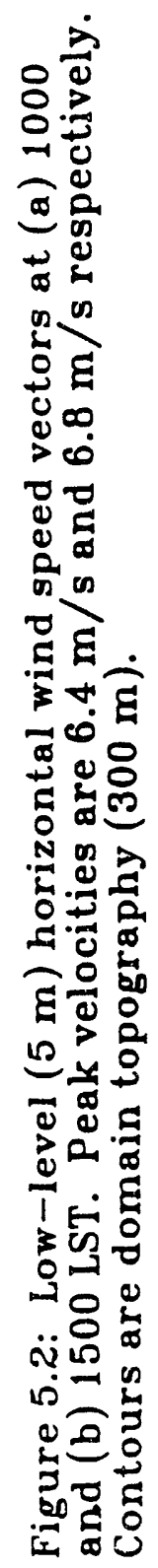
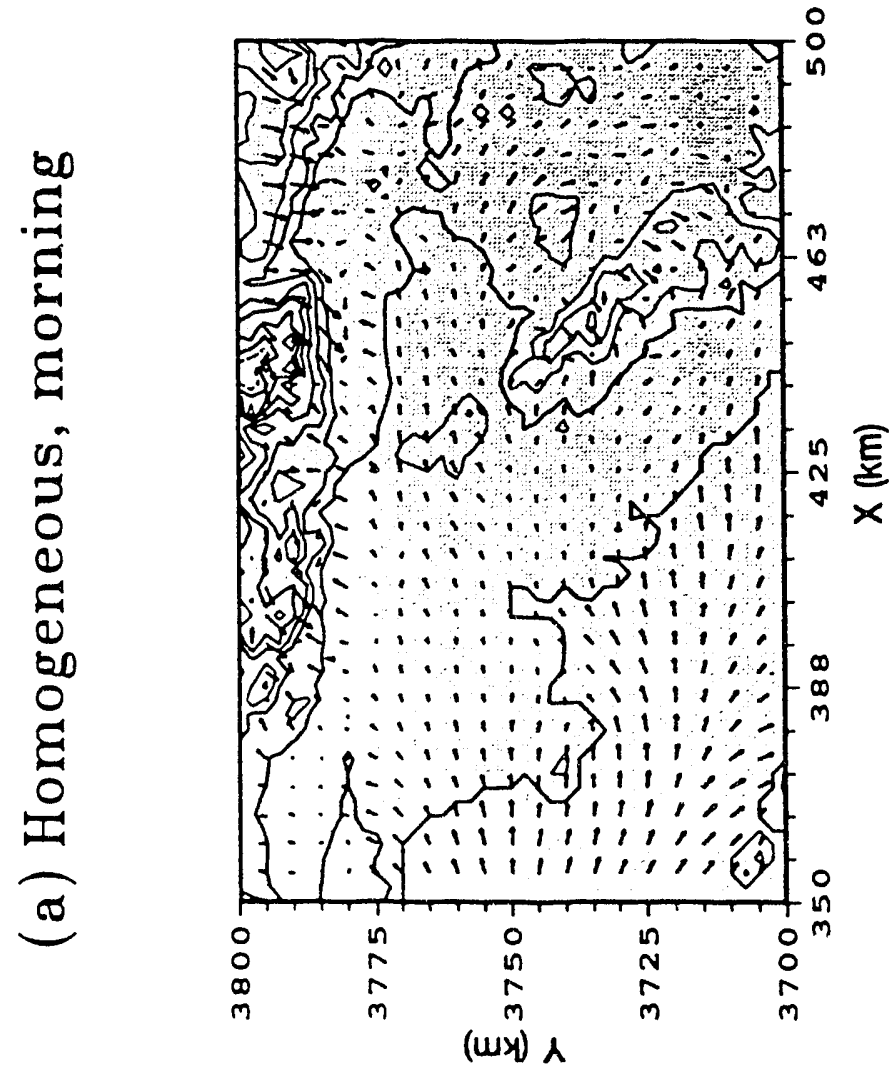

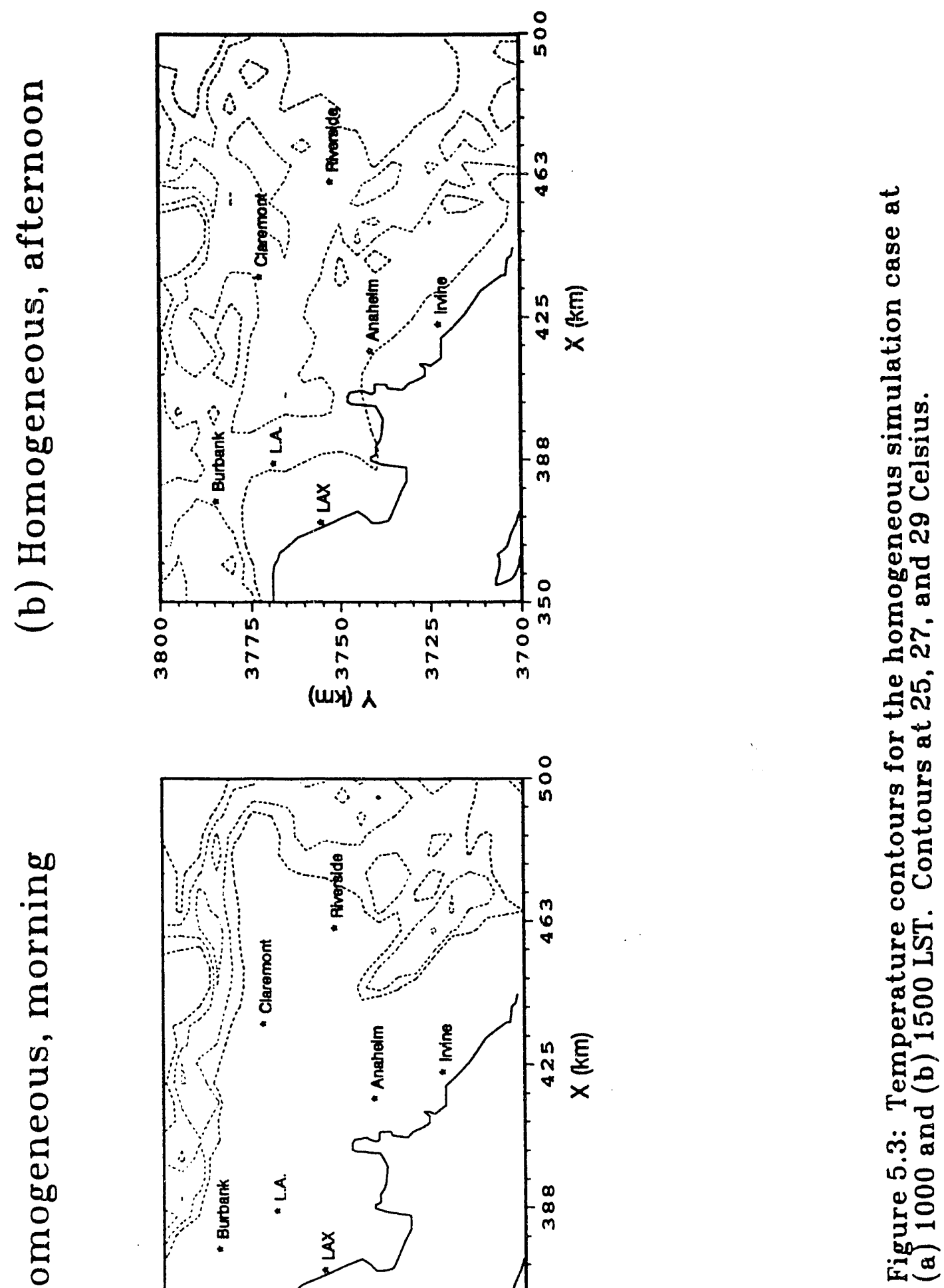

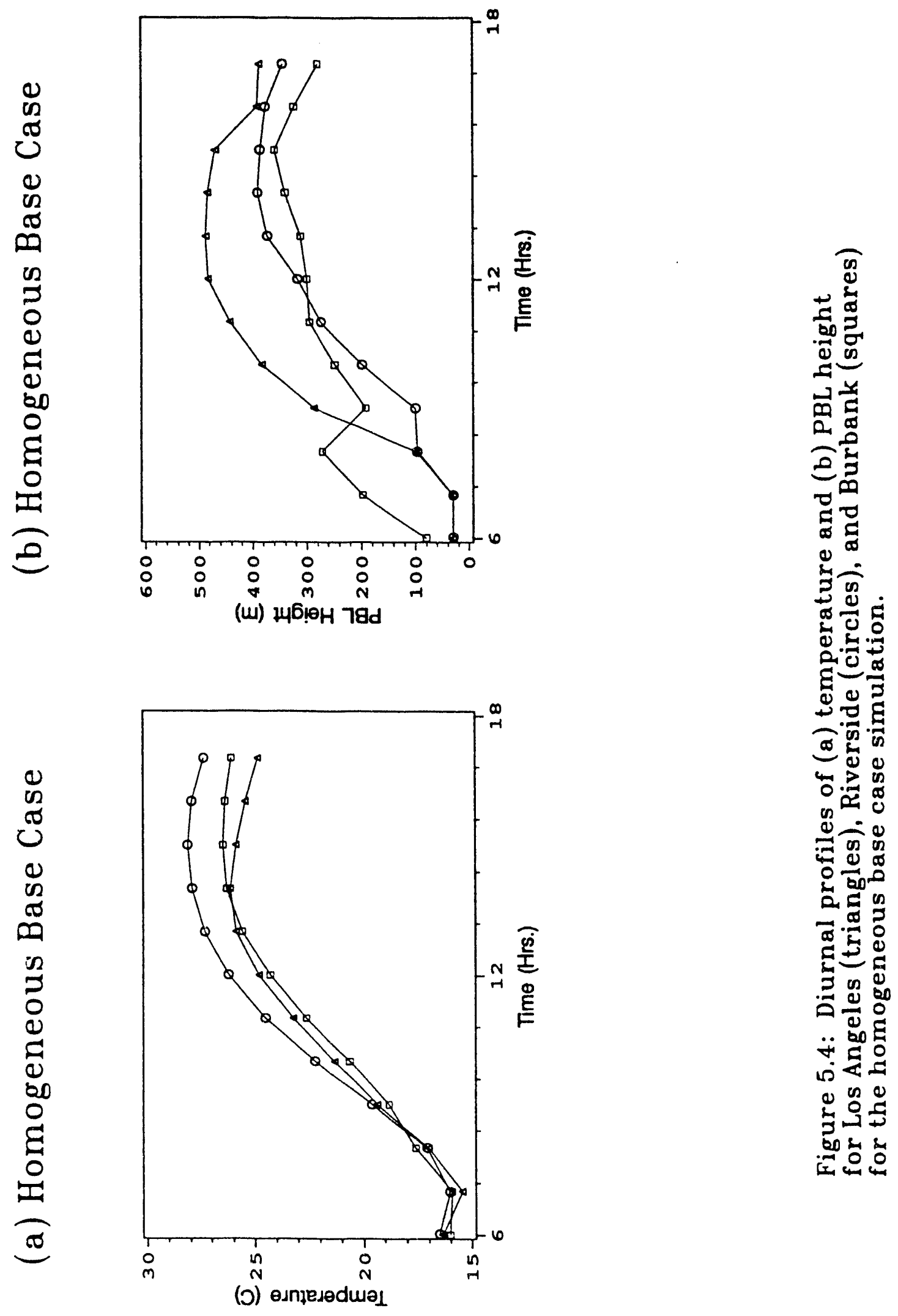


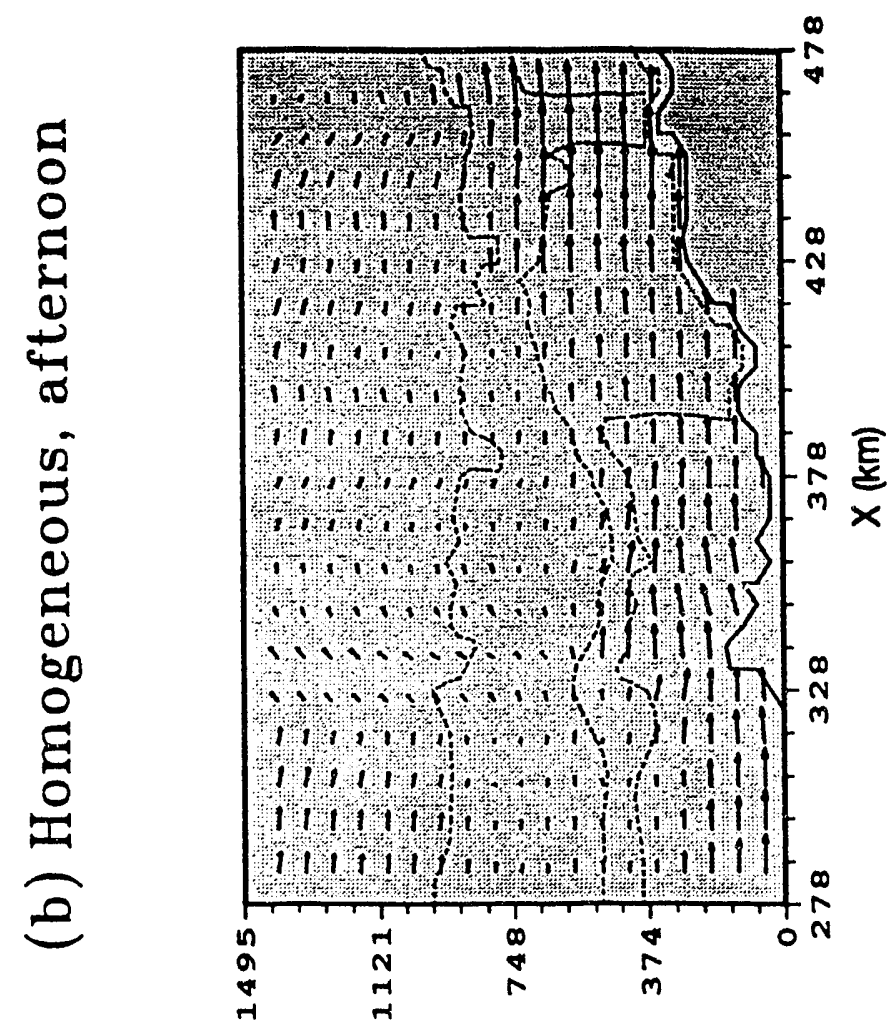

(w) $Z$

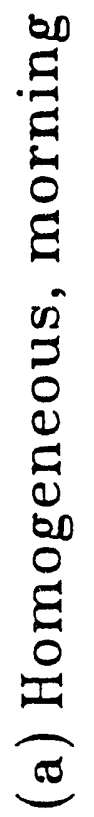
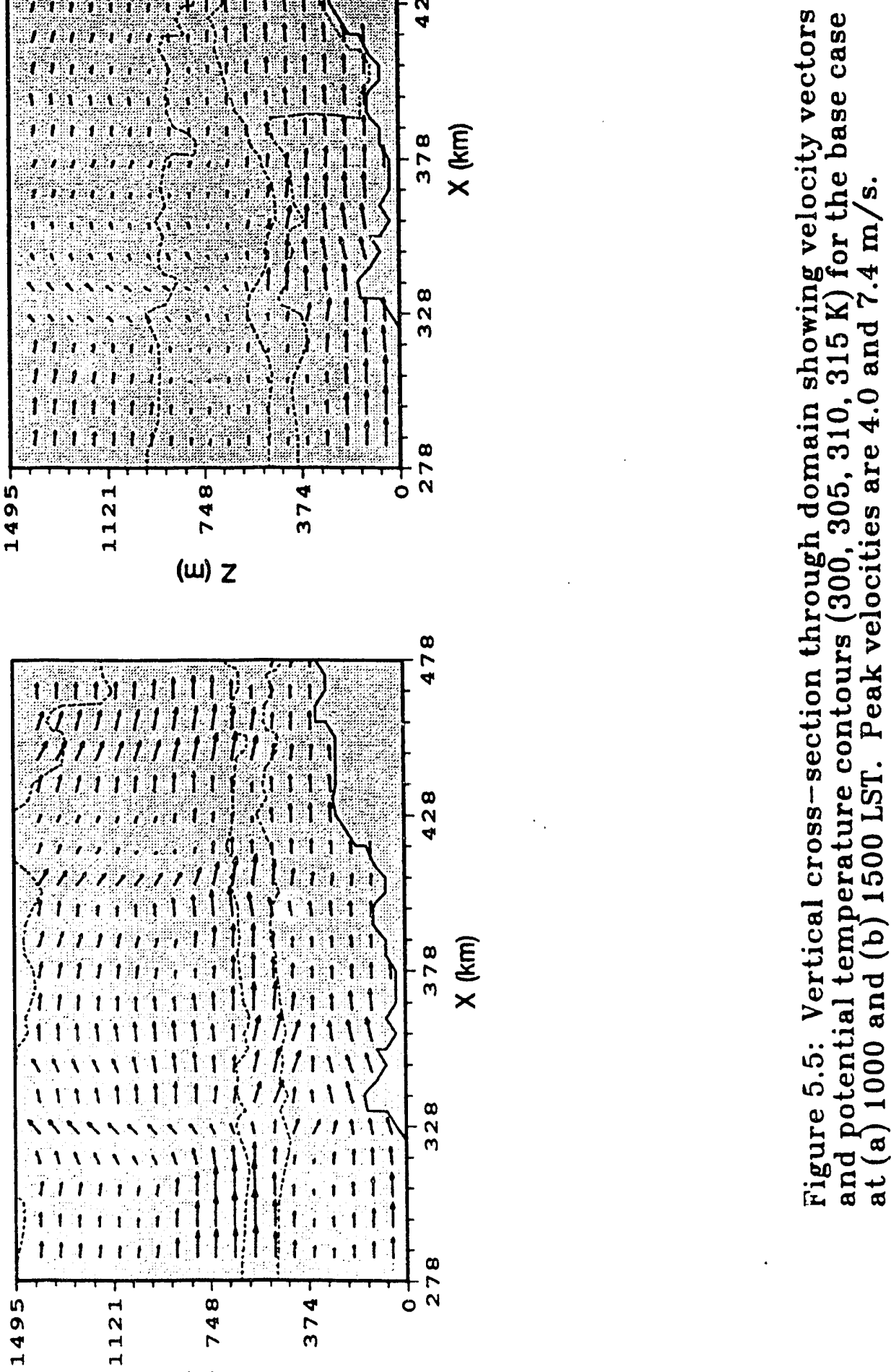

(w) Z 

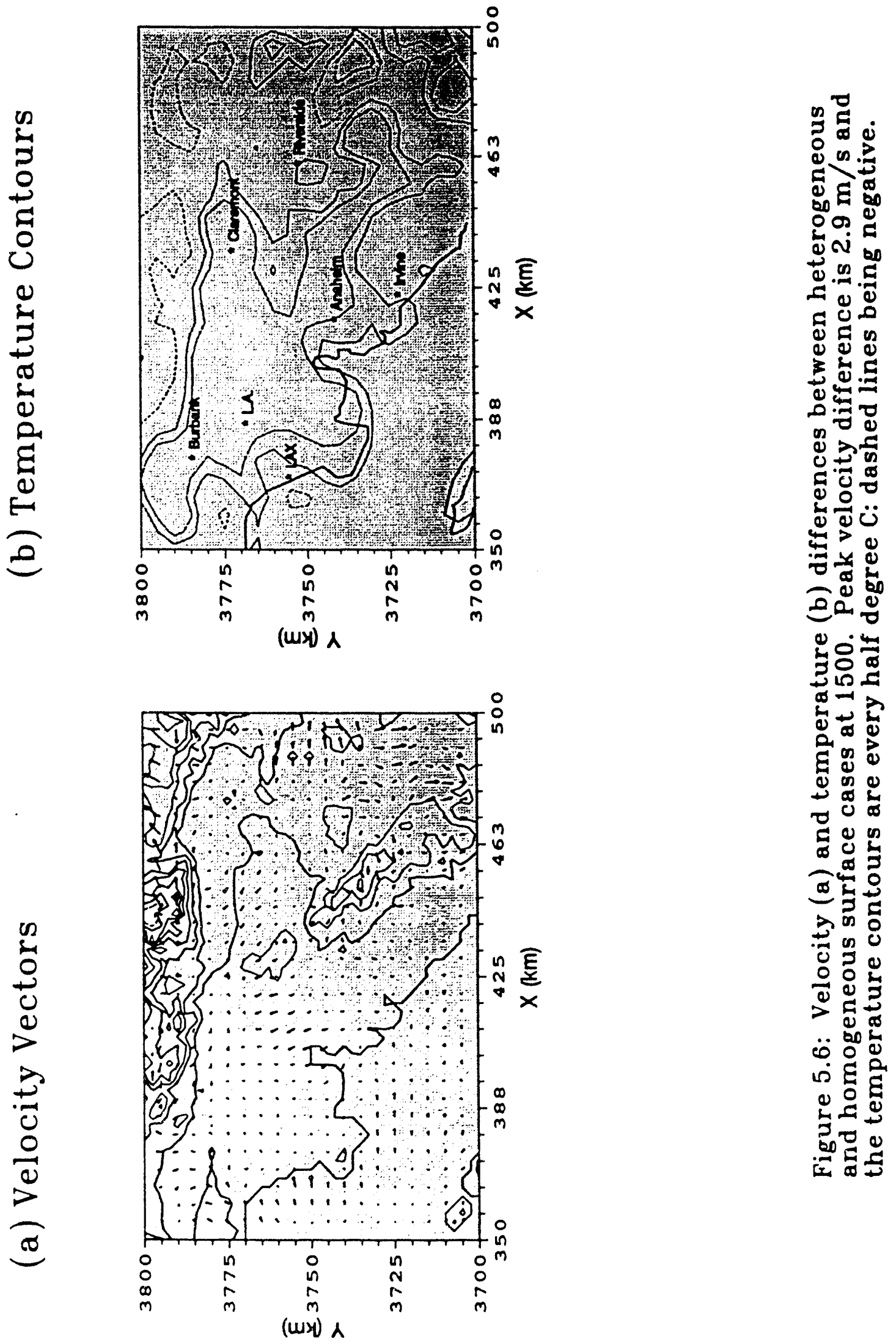


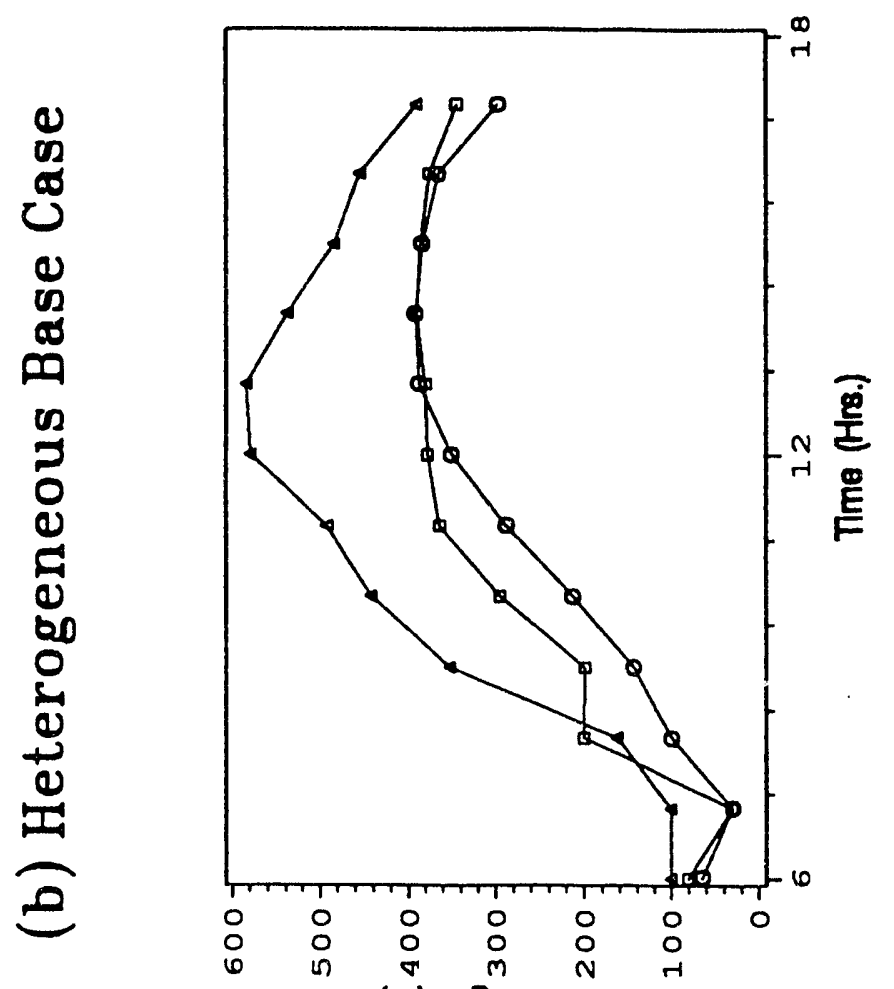

(w) 446 !० $78 d$
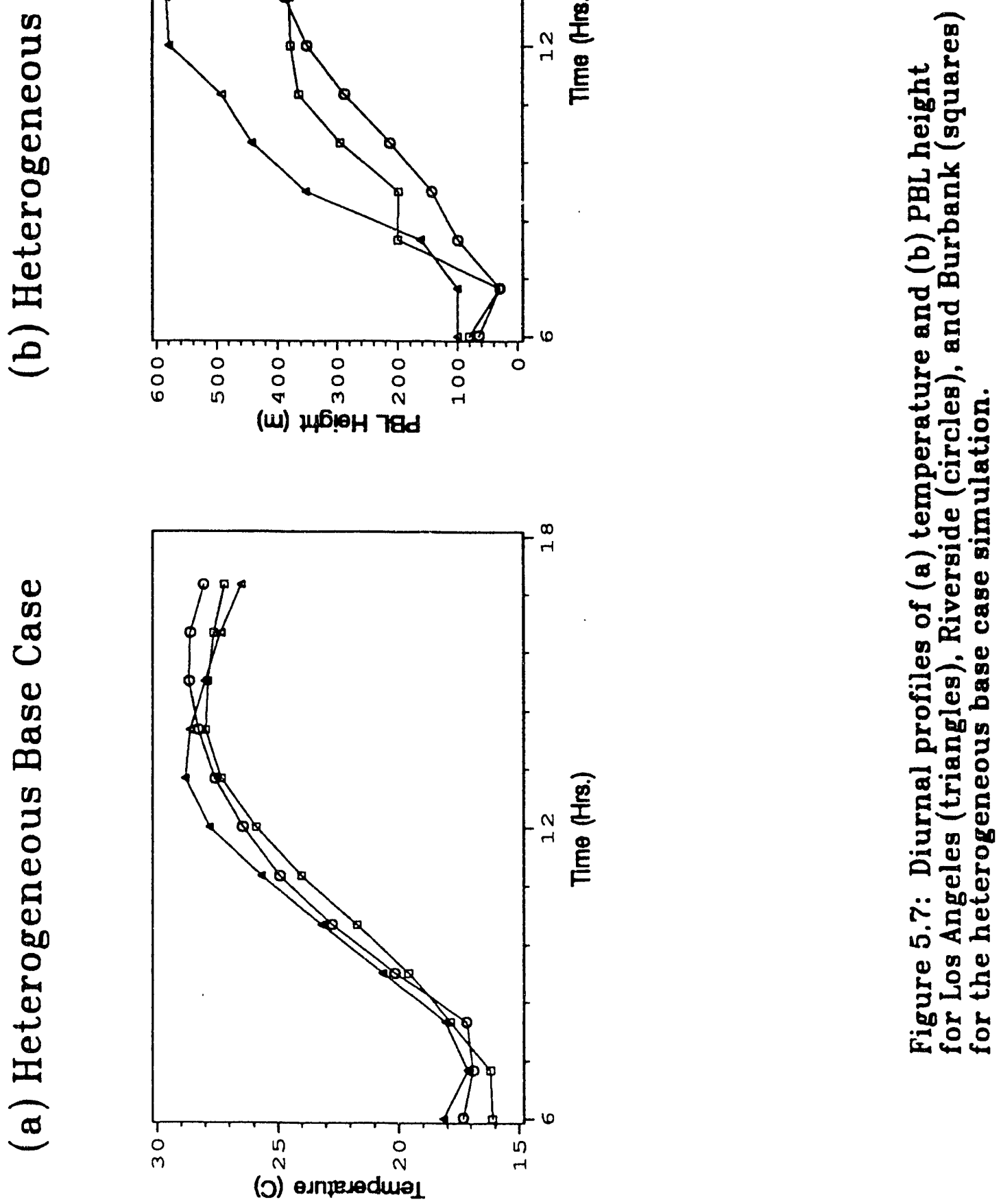

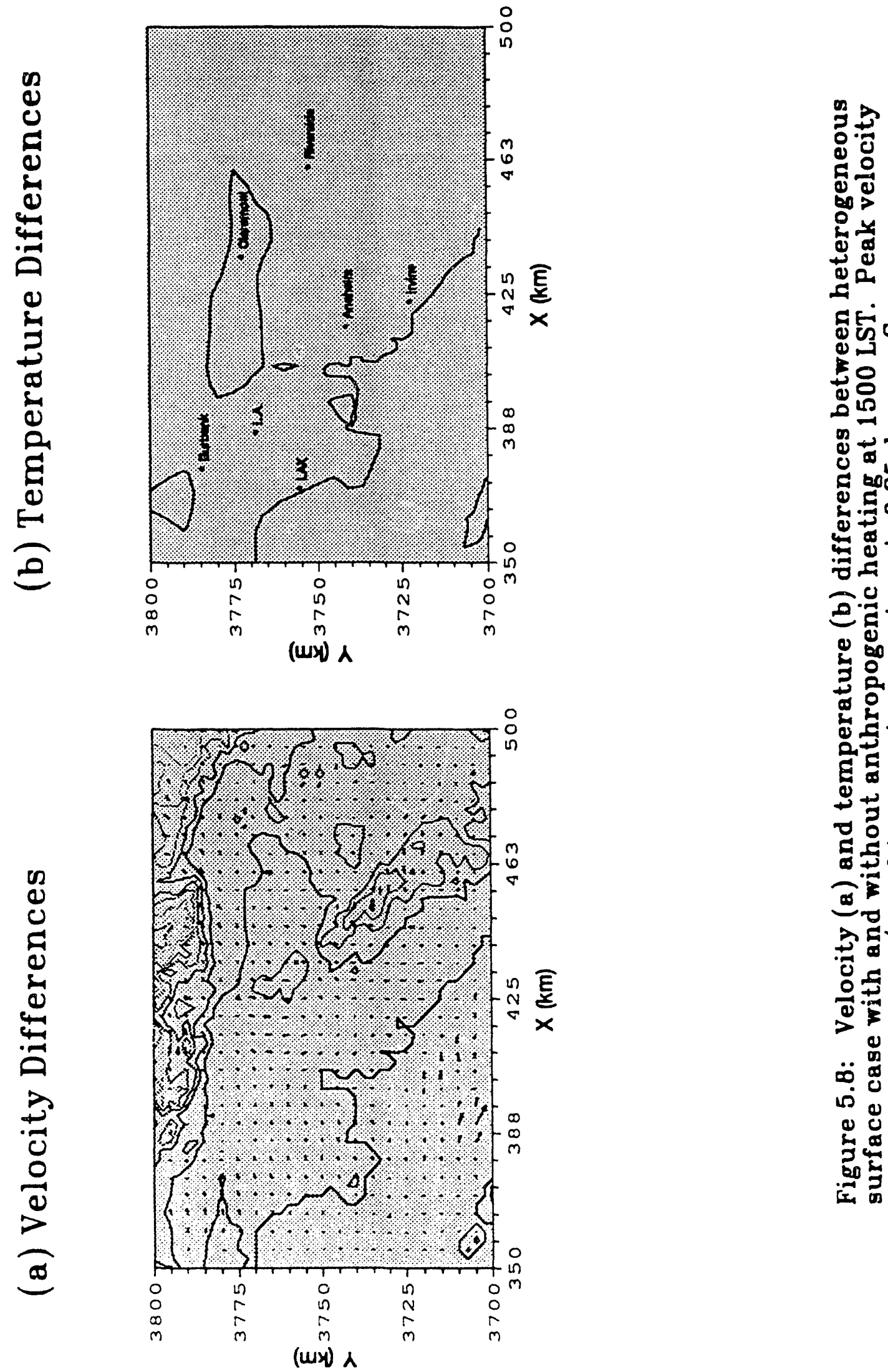

ธำำ 잉 ㅇㅇㅇ ๑ ठั द . Ð ซู 을웅 동영 4 a 군은 늘 은 동 홍 † 동 홍 क D 홍 ०

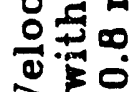
$>$. ம เi ๑ क 눈 


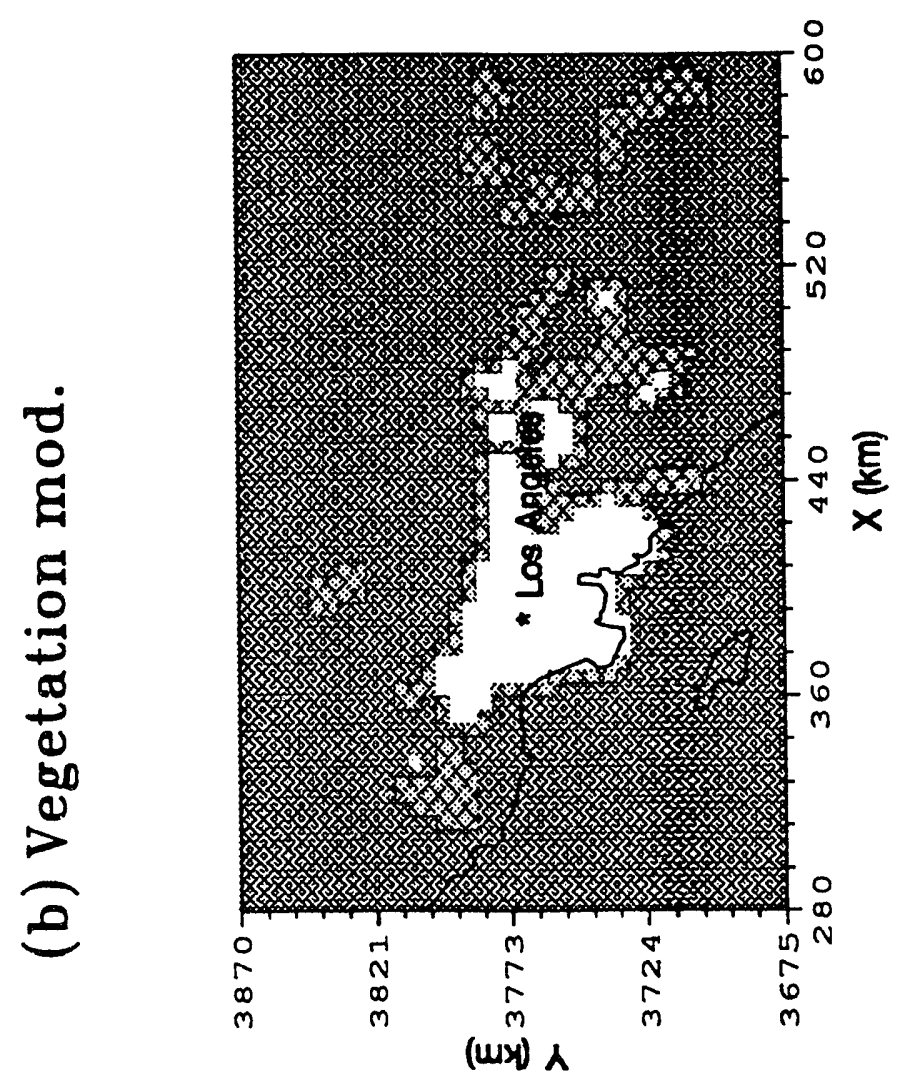

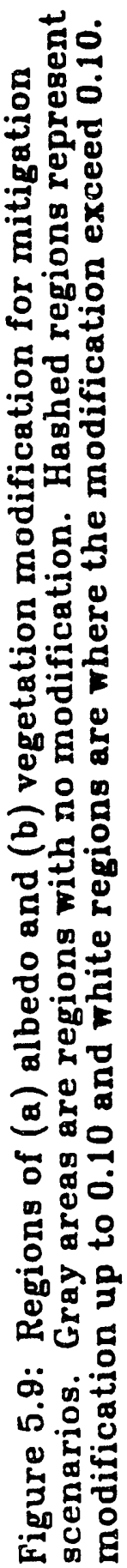

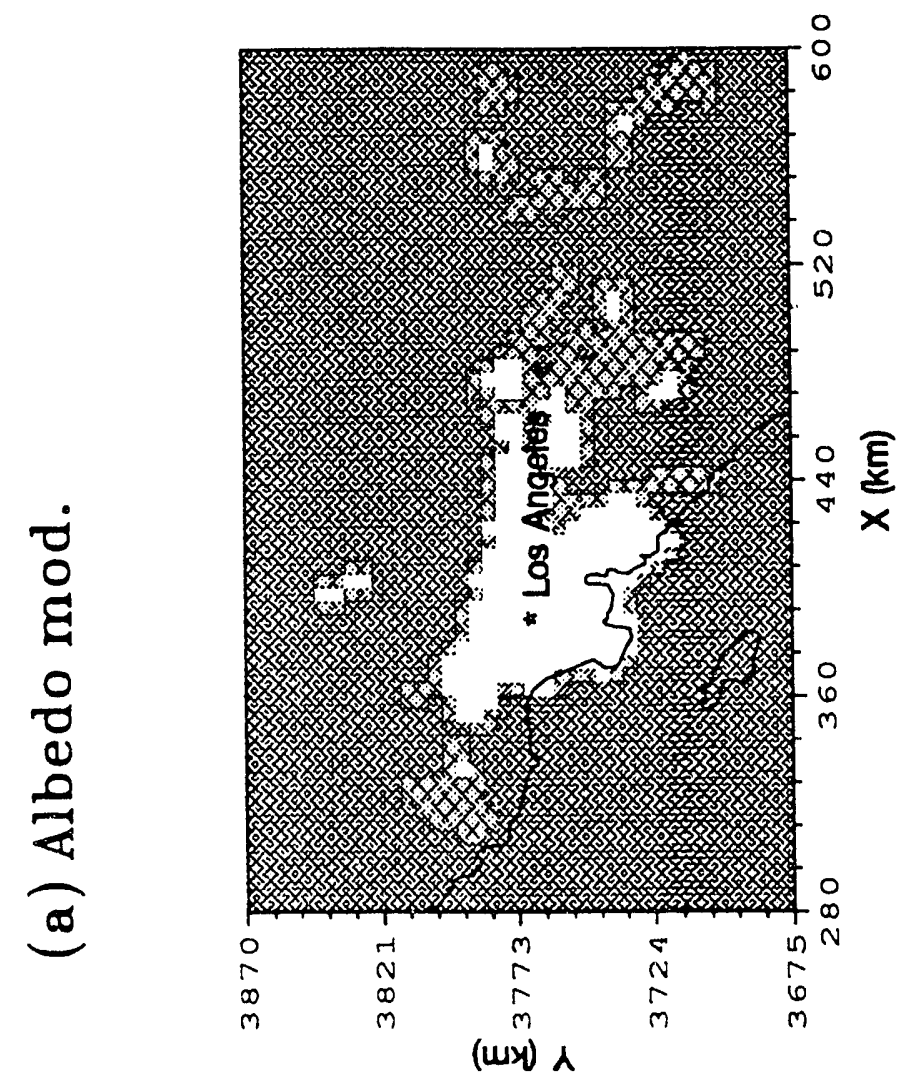


5
0
0
0
0
0
0
0
0
0
0
0
0
0
0
0
0
0
0
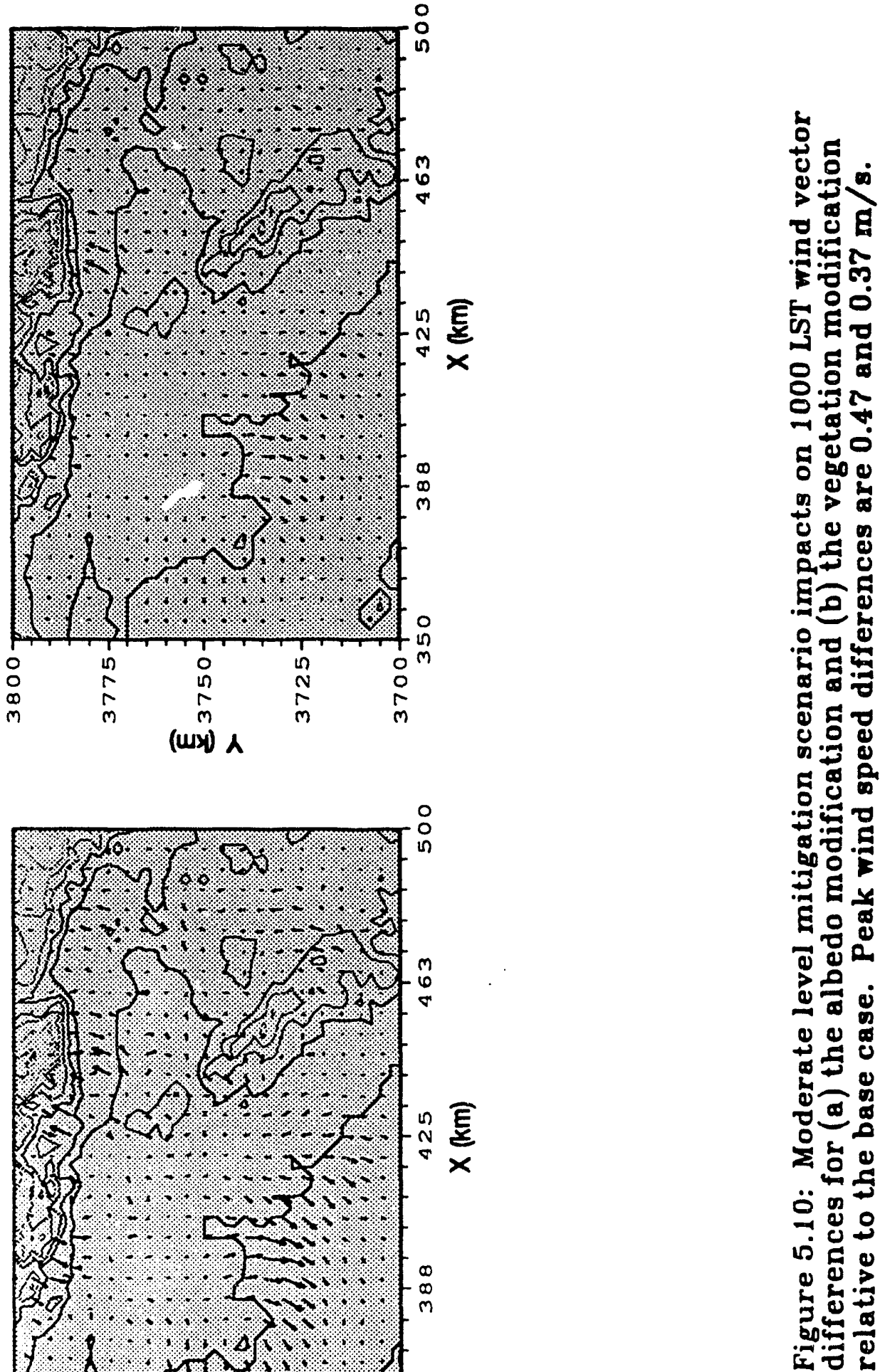

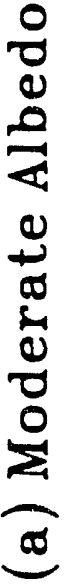

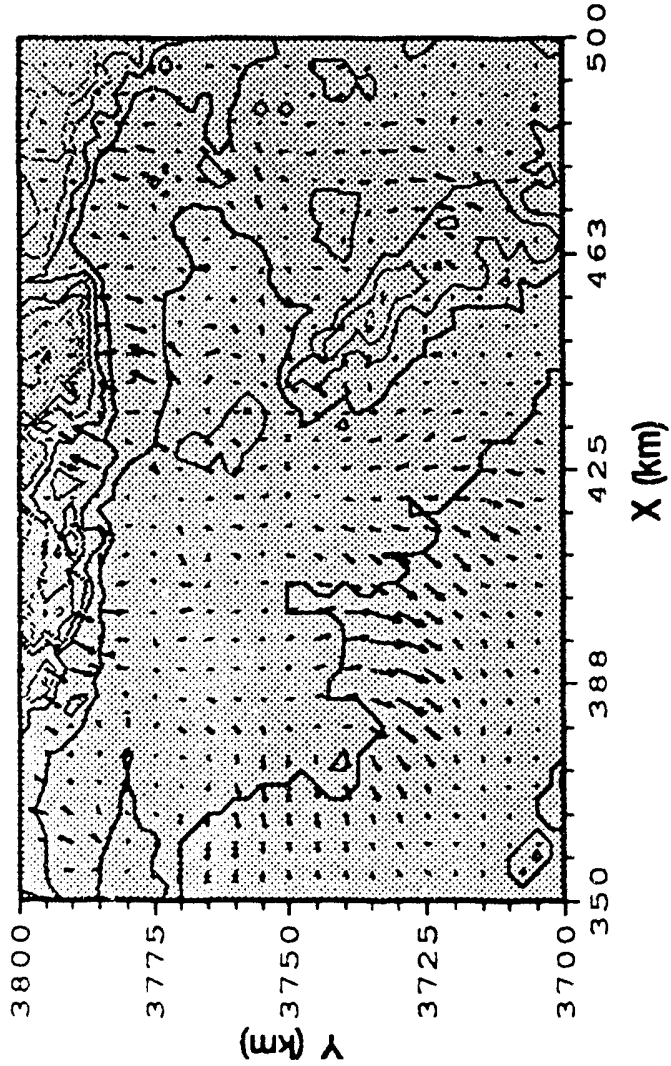



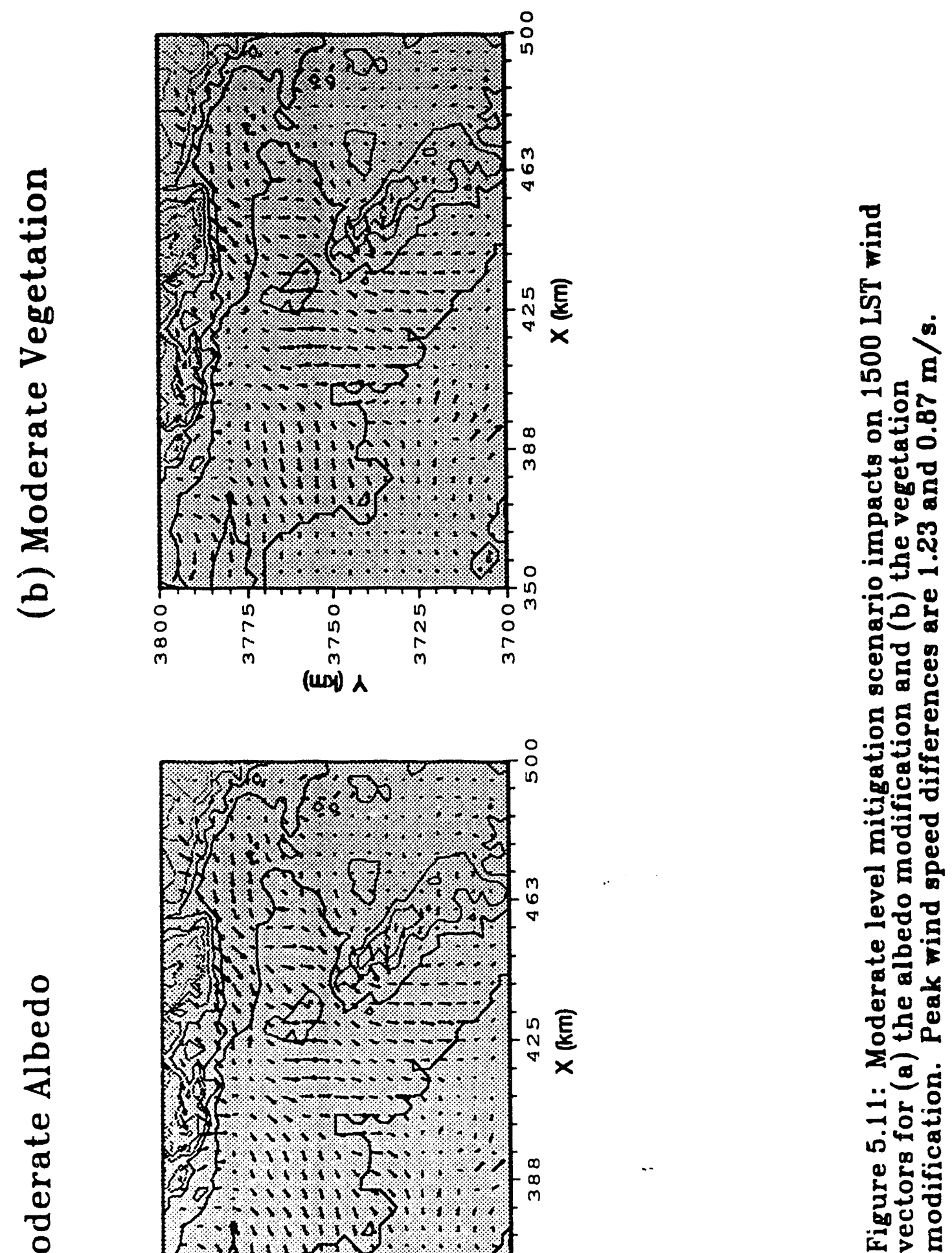

0
0
0
0
0
0
0
0
0
0
0
0
0
0
0

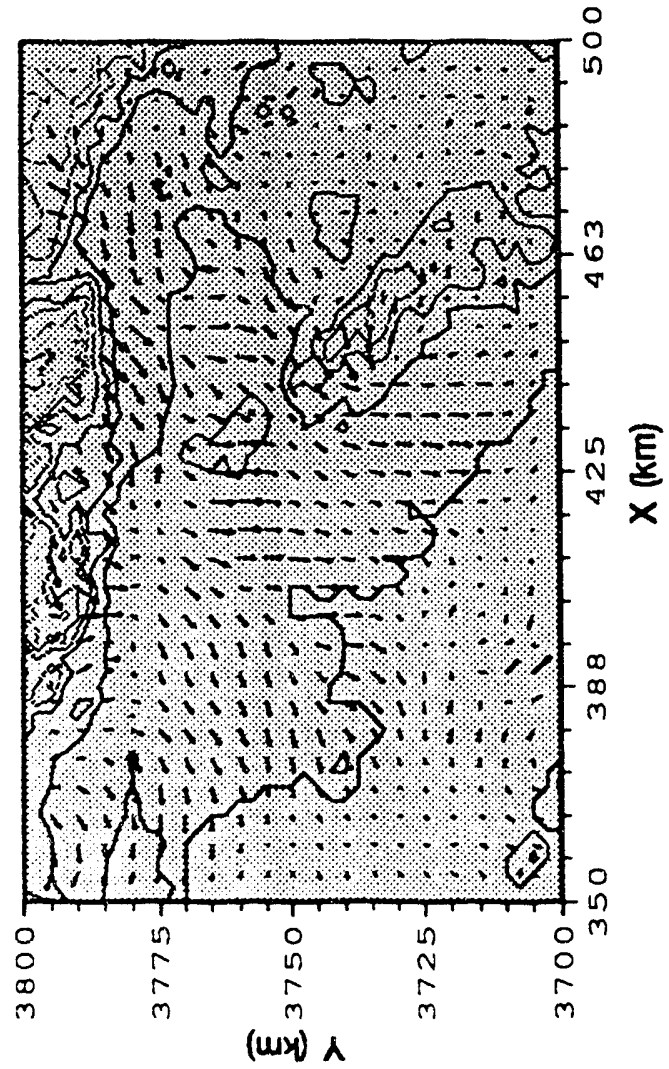




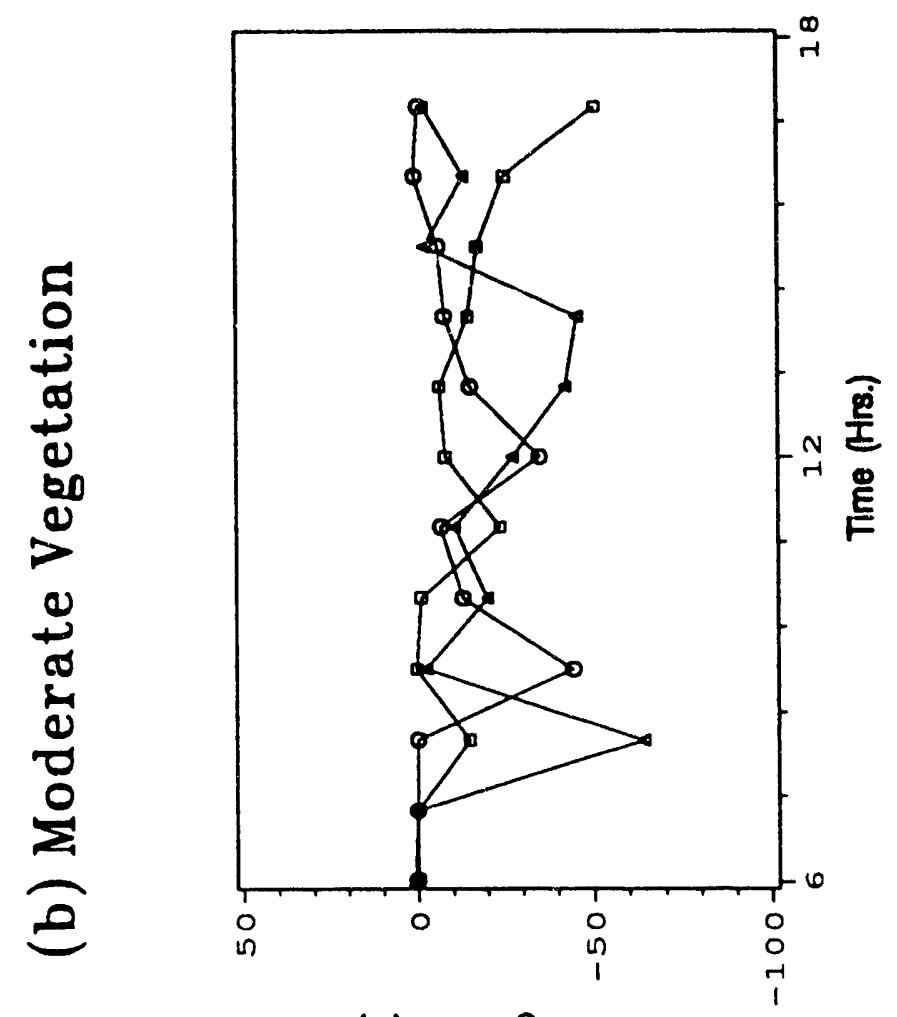

(w) W

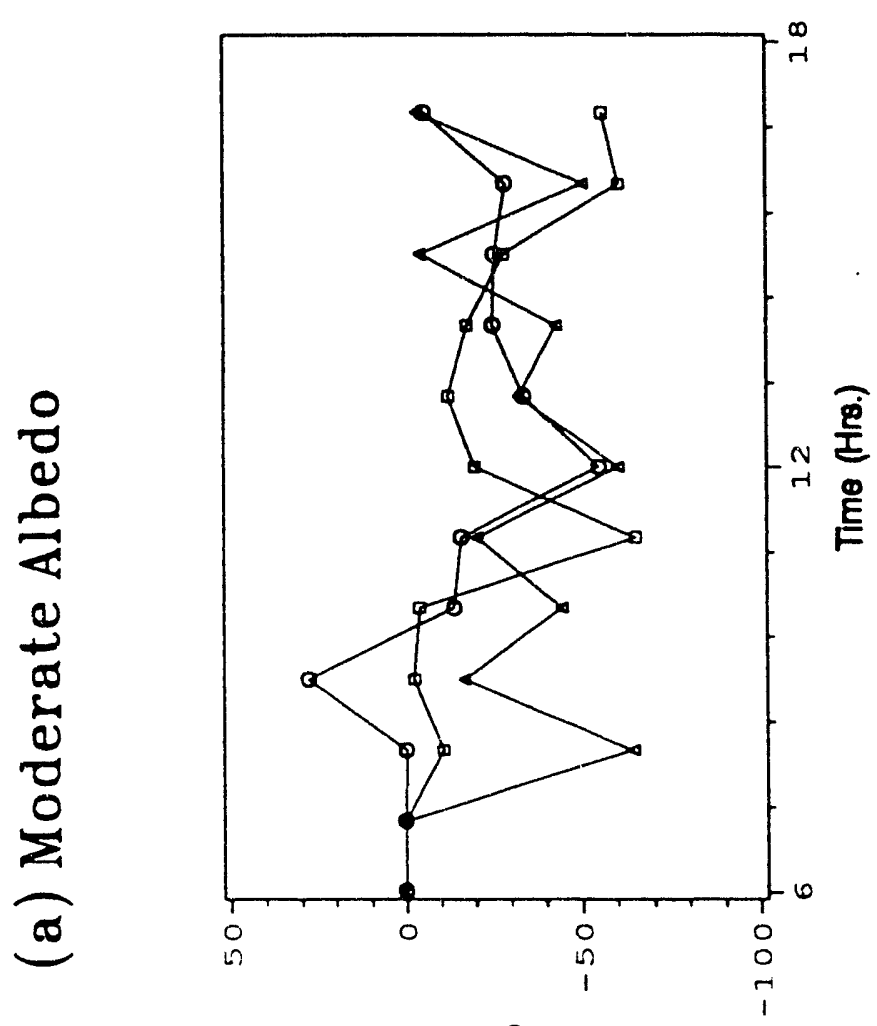

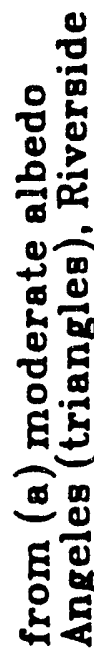

웅

홍

3 능

w

思

등

도

ตี

ơ

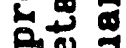

i $\infty$

ช 0

$+>$

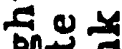

(1)

농

ต总

回最

을

10

的要

Ш

bo

ธิ

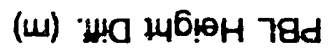




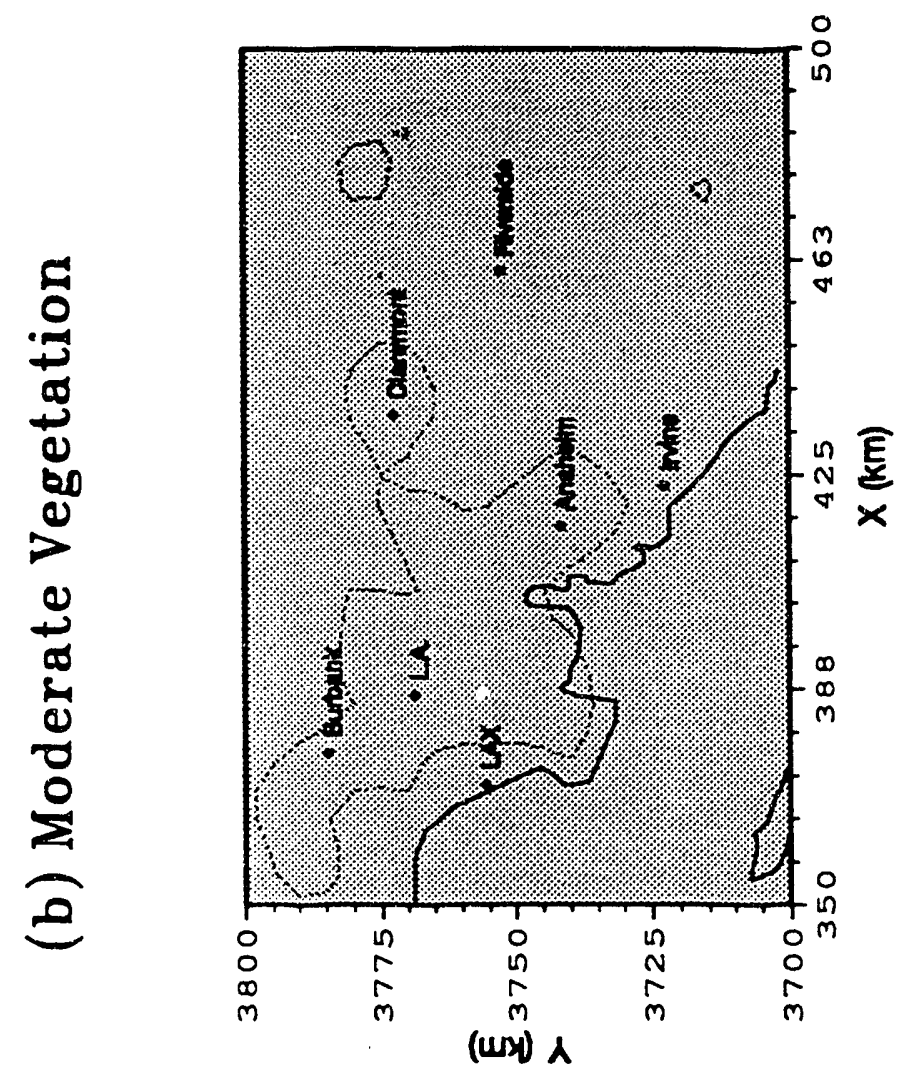

ฮี

茴男

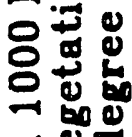
동뭉 o 엉현 مِق

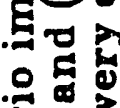
5 뗭뭉 눙
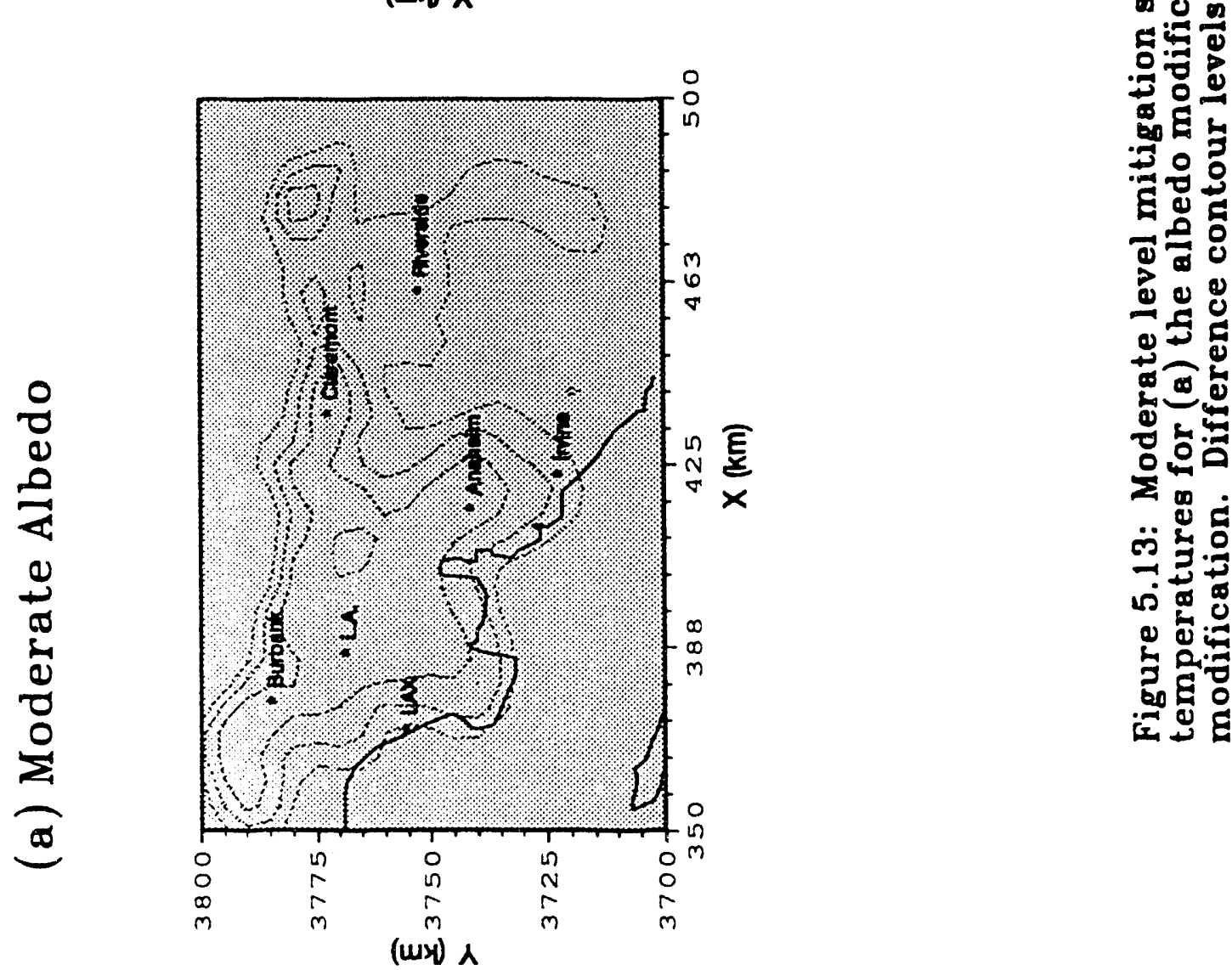

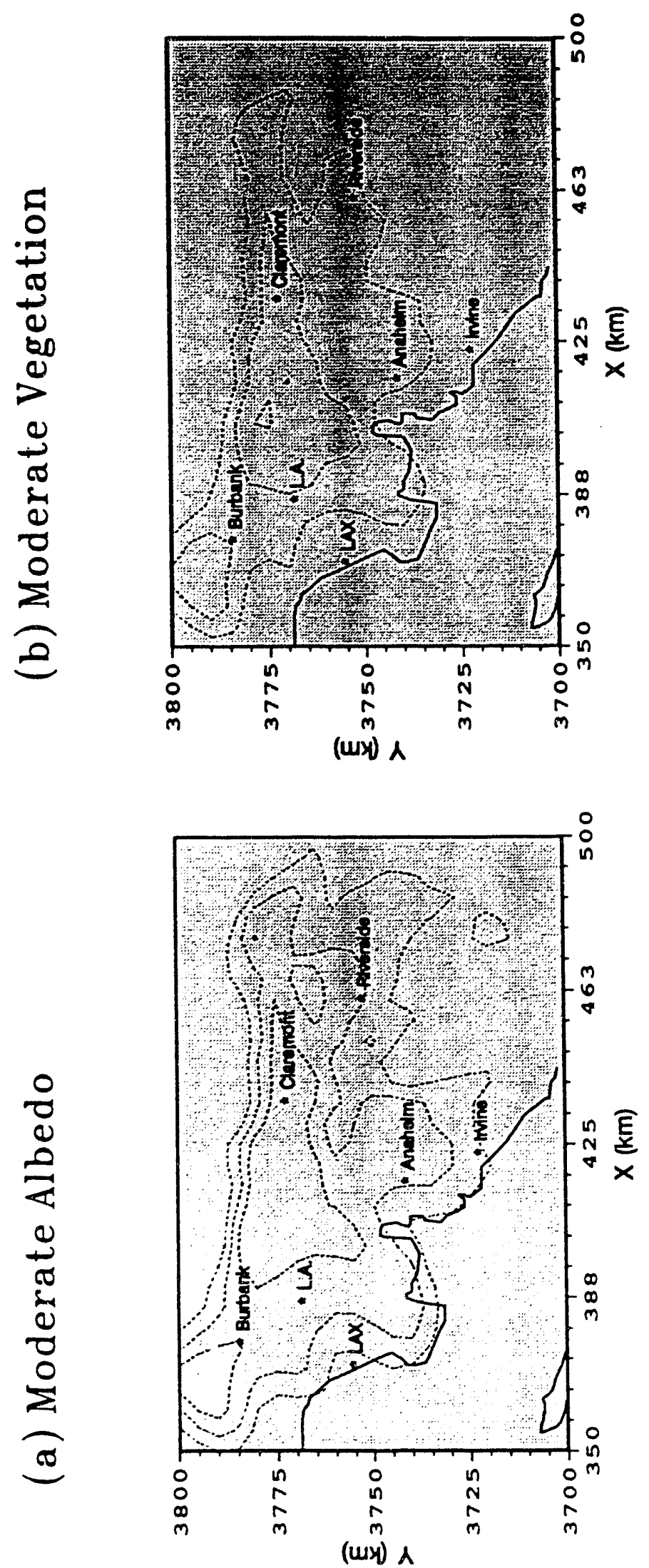

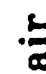

is

도웜용

ธ

o io

苍造

का

E्eo

등형 궁

우

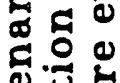

ฮี่

ถู ฮ

동

品

品

$=0$ ?

로웡

힝

ప 0

옹

Ш

包

웅영

$\sum$ 的

$\ddot{4} 3$

ज平

ง क

bo 

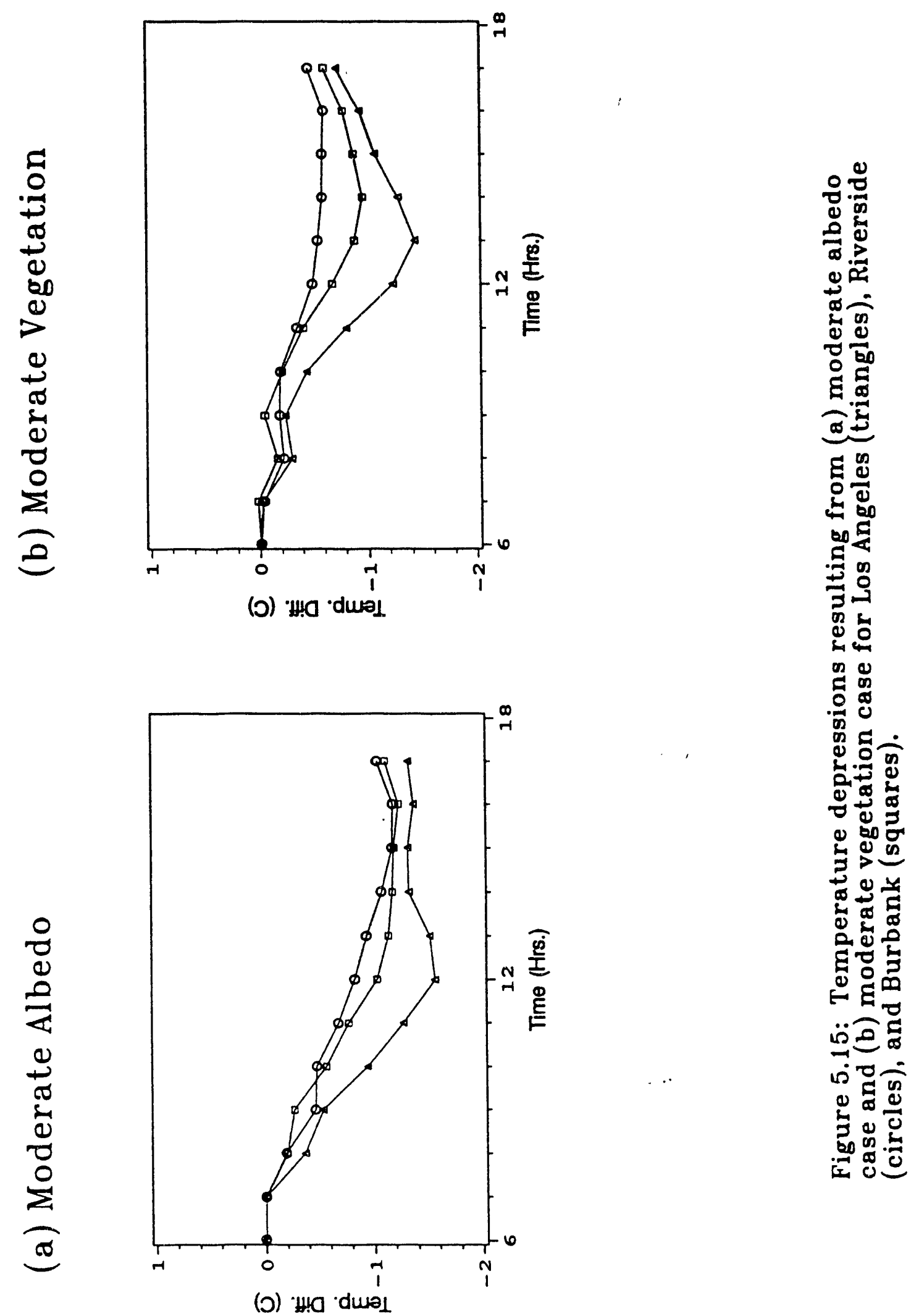


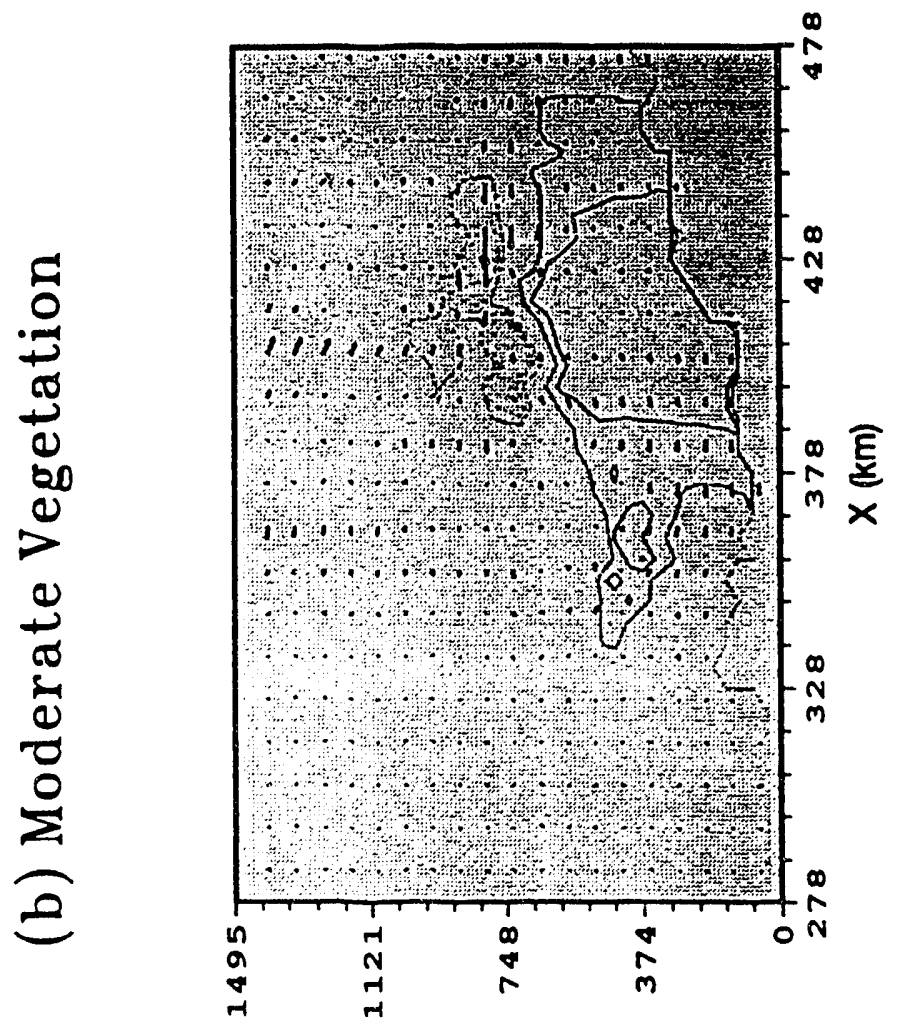

(w) Z
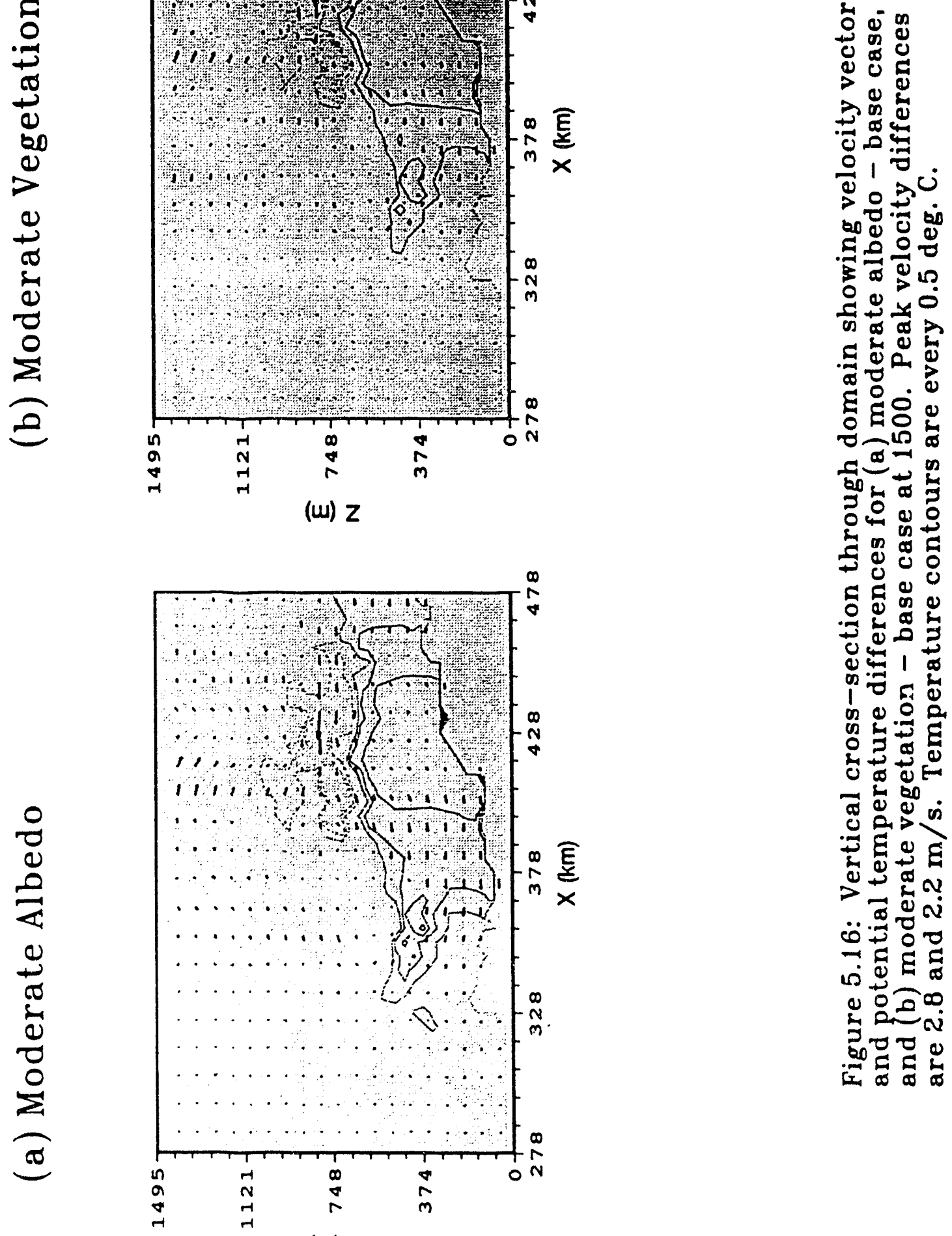

(w) Z 


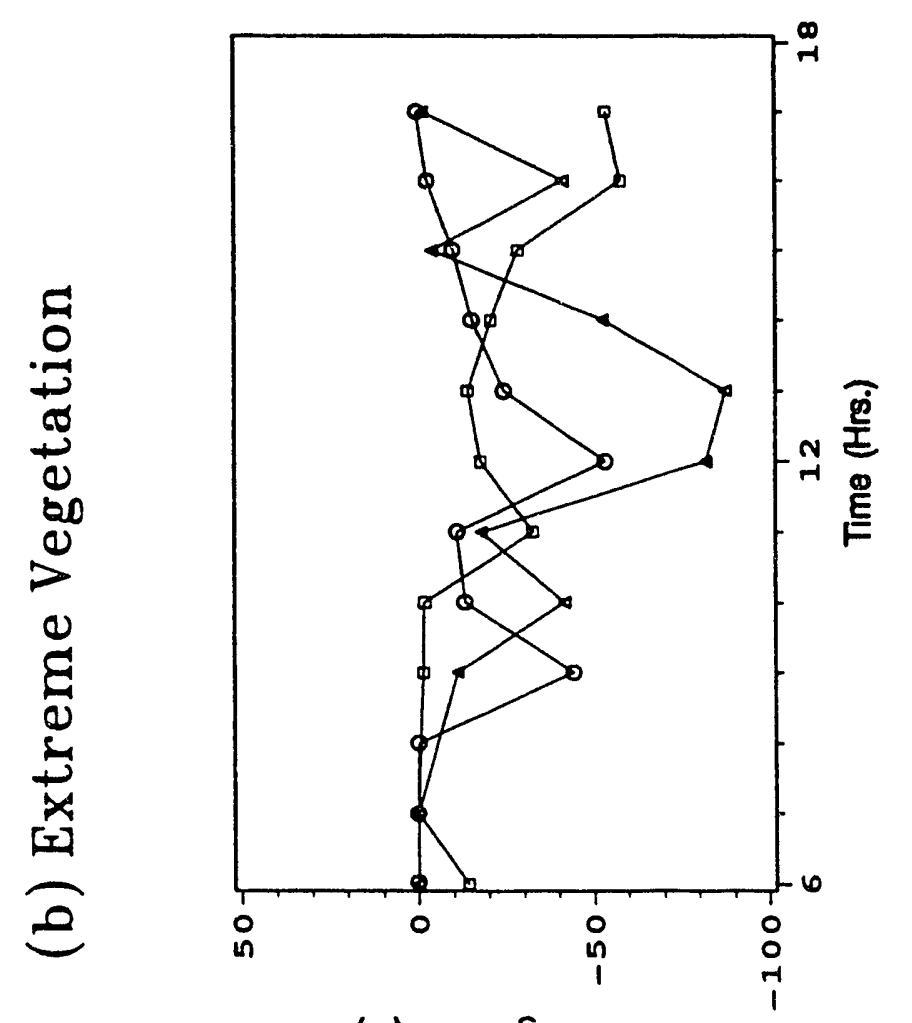

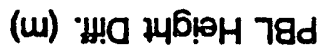

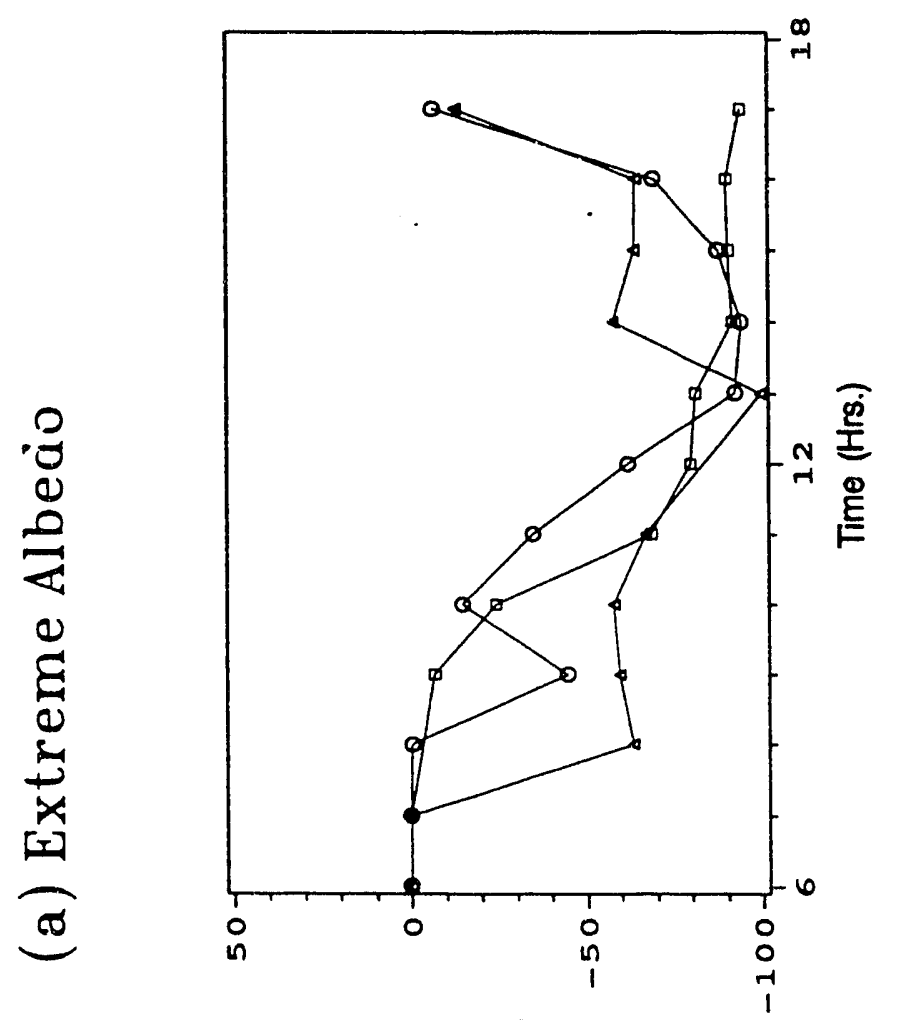

势

告

o :

究 号

क 동

․ㅜㄹ

คิ

ชั

$+8$

\& $>$

.

동

西喜占

吕希

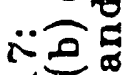

10

in

๑ ฮ

马o on

东它

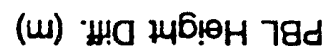




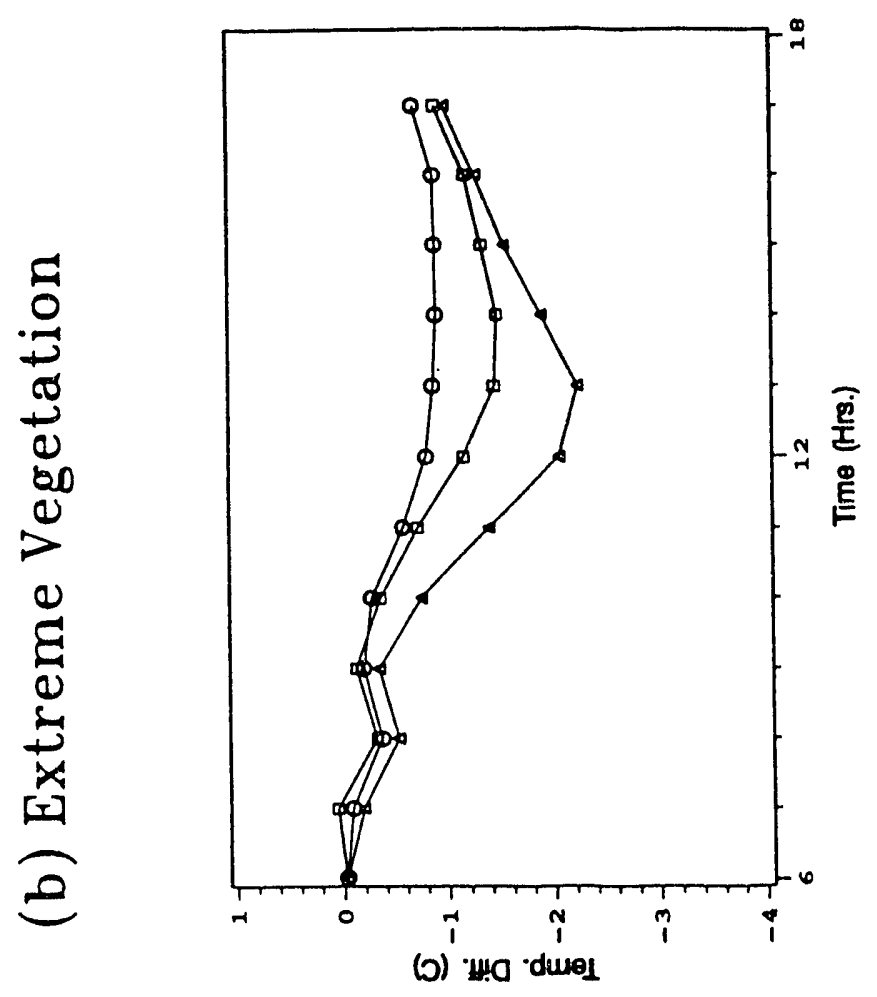

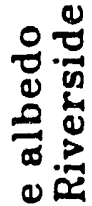

ฉัต

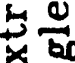

d)

Ð

옹

告

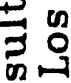

of

웅

过

कึ

\%

등

용

$0 \stackrel{5}{\exists}$

00

$\Rightarrow$

$\exists>$

。

¿

눈

車冓

F d

ه尺

م

๑)

700

का

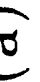



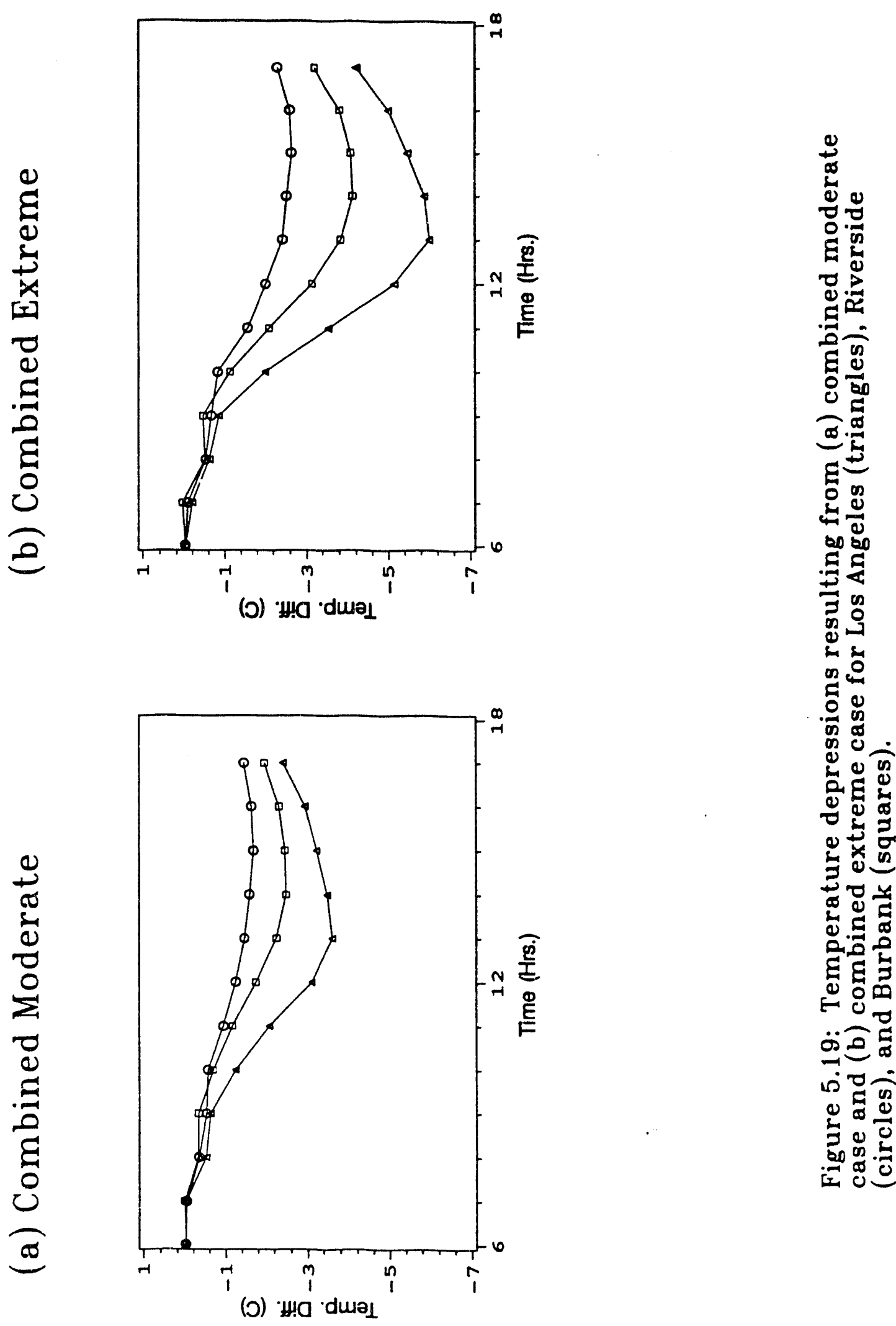


\section{REFERENCES}

Akbari, H., S. Bretz, J. Hanford, A. Rosenfeld, D. Sailor, H. Taha, and W. Bos, 1992. "Monitoring Peak Power and Cooling Energy Savings of Shade Trees and White Surfaces in the Sacramento Municipal Utility District (SMUD) Service Area," Final Report prepared for California Institute for Energy Efficiency and Sacramento Municipal Utility District. Lawrence Berkeley Laboratory report LBL-33342.

Akbari, H., Y.J. Huang, P. Martien, L. Rainer, A. Rosenfeld, and H. Taha, 1988. "The Impact of Summer Heat Islands on Cooling Energy Consumption and Global $\mathrm{CO}_{2}$ Concentration," in Proceedings of ACEEE 1988 Summer Study on Energy Efficiency in Buildings, Vol. 5, pp. 11-23, Asilomar CA, August.

Akbari, H, A. Rosenfeld, and H. Taha, 1989. "Recent Developments in Heat Island Studies: Technical and Policy," in Proceedings of the Workshop on Saving Energy and Reducing Atmospheric Pollution by Controlling Summer Heat Islands, Lawrence Berkeley Laboratory report LBL-27872.

Akbari, H., Rosenfeld, A.H., and Taha, H., 1990. "Summer Heat Islands, Urban Trees, and White Surfaces," in Proceedings of American Society of Heating, Refrigeration, and Air Conditioning Engineers, Atlanta, Georgia February. Also, Lawrence Berkeley Laboratory Report LBL-28308.

Andre, J.-C., and Blondin, C., 1986. "On the Effective Roughness Length for use in Numerical Three-Dimensional Models," Boundary-Layer Meteorology, Vol. 35, pp. 231-245.

Arnfield, J.A., 1982. "An Approach to the Estimation of the Surface Radiative Properties and Radiation Budgets of Cities," Phys. Geog., Vol. 3, pp. 97-122.

Arritt, R.W. 1985. "Numerical Studies of Thermally and Mechanically Forced Circulations Over Complex Terrain", Cooperative Institute for Research in the Atmosphere, Colorado State University, Fort Collins, Colorado.

Atkinson, R., 1988. "Atmospheric Transformations of Automotive Emissions," in Air Pollution, the Automobile and Human Health, eds. A.Y. Watson, R.R. Bates, and D. Kennedy, National Academy Press, Washington, D.C. 435 pp.

Atwater, M.A., 1972. "Thermal Effects of Urbanization and Industrialization in the Boundary Layer," Boundary-Layer Meteorology, Vol. 3, pp. 229-245. 
Avissar, R., and Mahrer, 1988. "Mapping Frost-Sensitive Areas with a ThreeDimensional Local-Scale Numerical Model. Part I: Physical and Numerical Aspects," Journal of Applied Meteorology, Vol. 27, pp. 400-413.

Avissar, R. and Pielke, R.A. 1989. "A Parameterization of Heterogeneous Land Surfaces for Atmospheric Numerical Models and Its Impact on Regional Meteorology", Monthly Weather Review, Vol. 117, pp. 2113-2136.

Bennet, M. and A.E. Saab, 1982. "Modeling of the Urban Heat Island and its Interaction with Pollutant Dispersals," Atmospheric Environment, Vol. 16, No. 8, pp. 1797-1822.

Birdsall, B.E., W.F. Buhl, R.B. Curtis, A.E. Erdem, J.H. Eto, J.J. Hirsch, K.H. Olson, and F.C. Winkelmann, 1985. "The DOE-2 Computer Program for Thermal Simulation of Buildings," in D. Hafemeister, H. Kelly and B. Levi (Eds.), Energy Sources: Conservation and Renewables, Appendix E, American Institute of Physics Conference Proceedings, No. 135, pp. 642-649.

Bornstein, R.D., 1975. "The Two-Dimensional URBMET Urban Boundary Layer Model," Journal of Applied Meteorology, Vol. 14, pp. 1459-1477.

Bornstein, R.D., 1984. "Urban climate models: Nature, Limitations and Applications," Preprints from WMO Technical Conference on Urban Climatology and its Application with Special Regard to Tropical Areas, Mexico City.

Bornstein, R.D., 1989. "Nature, Limitations, and Applications of Urban Climate Models," in Proceedings of the workshop on saving energy and reducing atmospheric pollution by controlling summer heat islands, Berkeley, California, pp. 124-149.

Bornstein, R.D., U. Pechinger, R. Miller, S. Klotz, and R. Street, 1987. "Modeling the Polluted Coastal Urban Environment," EPRI EA-5091, Vol. 1, Final Report, Research Project 1630-13.

Brest, C.L., and S.N. Goward, 1987. "Deriving Surface Albedo Measurements from Narrow Band Satellite Data," International Journal of Remote Sensing, Vol. 8, No. 3, pp. 351-367.

Bretz, S., H. Akbari, A. Rosenfeld, and H. Taha, 1992. "Implementation of SolarReflective Surfaces: Materials and Utility Programs," Lawrence Berkeley Laboratory Report LBL-32467.

Brundl, W., M. Kniep, and H. Mayer, 1985. Untersuchung des Einflusses von Bebauung und Bewuchs auf das Klima und die lufthygienischen Verhältnisse in bayerischen Großstadten. Stadtklima Bayern, Daten aus dem Mënets Bodenniveau in München vom 1.7.1984 bis 31.12.1984. 
Buffington, D.E., 1979. "Economics of Landscaping Features of Conserving Energy in Residences," in Proceedings of Florida State Horticulture Society, Vol. 92, pp. 216-220.

Carlson, T.N. and F.E. Borland, 1978. "Analysis of Urban-Rural Canopy using a Surface Heat Flux/Temperature Model," Journal of Applied Meteorology, Vol. 17, pp. 998-1012.

Carlson, T.N., R. Gillies, and B. Lynn, 1989. Unpublished model description and user's manual. Penn. State University.

Chameides, W.L., R.W. Lindsay, J. Richardsen, and C.S. Kiang, 1988. "The Role of Biogenic Hydrocarbons in Urban Photochemical Smog: Atlanta as a Case Study," Science, Vol. 241, pp. 1473-1475.

Chandler, T.J., 1965. The Climate of London, London, Hutchinson, 292 pp.

Changnon, S.A. 1976. "Inadvertent Weather Modifications." Water Resources Bulletin, Vol. 12, pp. 695-718.

Changnon, S.A., 1981. METROMEX, a Review and Summary, Edited by Stanley A. Changnon Jr., American Meteorological Society, Boston, MA.

Conner, C., 1985. "The $\mathrm{F}$ of Exterior Surface Absorptivity and Emissivity on Energy use in Residential Buildings," Unpublished report, Lawrence Berkeley Laboratory.

van Dop, H., 1983. "Terrain Classification and Derived Meteorological Parameters for Interregional Transport Models," Atmospheric Environment, Vol. 17, pp. 1099-1105.

Doran, J.C., Barnes, F.J., Coulter, R.L., Crawford, T.L., Baldocchi, D.D., Balick, L., Cook, D.R., Cooper, D., Dobosy, R.J., Dugas, W.A., Fritschen, L., Hart, R.L., Hipps, L., Hubbe, J.M., Gao, W., Hicks, R., Kirkham, R.R., Kunkel, K.E., Martin, T.J., Meyers, T.P., Porch, W., Shannon, J.D., Shaw, W.J., Swiatek, E., and C.D. Whiteman, 1992. "The Boardman Regional Flux Experiment," Bulletin American Meteorological Society, Vol. 73, No. 11, pp. 1785-1795.

Eichorn, J., R. Schrodin, and W. Zdunkowski, 1988. "Three-Dimensional Numerical Simulations of the Urban Climate," Beitr. Phys. Atmosph. , Vol. 61, pp. 187-203.

Forster, B., 1983. "Some Urban Measurements from Landsat Data," Photogrammetric Engineering \& Remote Sensing, Vol. 49, No. 12, pp. 1693-1707. 
Goward, S.N., G.D. Cruickshanks, and A.S. Hope, 1985. "Observed Relation between Thermal Emission and Reflected Spectral Radiance of a Complex Vegetated Landscape," Remote Sensing of Environment, Vol. 18, pp. 137-146.

Griggs, E.I., T.R. Sharp, and J.M. MacDonald, 1989. "Guide for Estimating Differences in Building Heating and Cooling Energy due to Changes in Solar Reflectance of a low-sloped Roof," Oak Ridge National Laboratory Report, ORNL-6527.

Guenther, A.B., R.K. Monson, and R. Fall, 1991. "Isoprene and Monoterpene Emission Rate Variability: Observations with Eucalyptus and Emission Rate Algorithm Development," Journal of Geophysical Research, Vol. 96, pp. 799810.

Hjelmfelt, M.R. 1982. "Numerical Simulation of the Effects of St. Louis on Mesoscale Boundary-Layer. Airflow and Vertical Air Motion: Simulations of Urban vs. Non-Urban Effects", Journal of Applied Meteorology, Vol. 21, pp. 1239-1257.

Honjo, T. and T. Takakura, 1986. "Analysis of Temperature Distribution of Urban Green Spaces using Remote Sensing Data," Journal of the Japanese Institute of Landscape Architecture, Vol. 49, No. 5, pp. 299-304.

Horie, Y., S. Sidawl, and R. Ellefsen, 1990. "Inventory of Leaf Biomass and Emission Factors for Vegetation in California's South Coast Air Basin," Final Report prepared for South Coast Air Quality Management District.

Hosler, C.L. and Landsberg, H.E. 1977. "The Effect of Localized Man-Made Heat and Moisture Sources in Mesoscale Weather Modification", in Energy and Climate, National Academy of Sciences, Washington, D.C.

Howard, Luke, 1833. The Climate of London, Deduced from Meteorological Observations, Made in the Metropolis, and at Various Places Around it, 2nd ed., London, Harvey and Darton, 3 volumes.

Huang, Y.J., H. Akbari, H. Taha, and A. Rosenfeld, 1987. "The Potential of Vegetation in Reducing Summer Cooling Loads in Residential Buildings," Journal of Climate and Applied Meteorology, Vol. 26, pp. 1103-1116.

Kalma, J.D. and Newcombe, K.J. 1976. "Energy Use in Two Large Cities: A Comparison of Hong Kong and Sydney, Australia", Environmental Studies, Vol. 9, pp. 53-64.

Karl, T.R., H.F. Diaz, and G. Kukla, 1988. "Urbanization: Its Detection and Effects in the United States Climate Record," Journal of Climate, Vol. 1, pp. 1099-1123. 
Kessler, R.C.; Eppel, D.; Pieike, R.A.; and McQueen, J. 1985. "A Numerical Study of the Effects of a Large Sandbar upon Se:-Breeze Development", Archives for Meteorology, Geophysics, and Bioclimatology, Vol. 34, pp. 3-26.

Kimura, F., and Takahashi, S. 1991. "The Effects of Land-Use and Anthropogenic Heating on the Surface Temperature in the Tokyo Metropolitan Area: A Numerical Experiment", Atmospheric Environment, Vol. 25B, No. 2, pp. 155164.

Kukla, G., J. Gavin, and T.R. Karl, 1986. "Urban Warming," Journal of Climate and Applied Meteorology, Vol. 25, pp. 1265-1270.

Kung, E.C., 1963. "Climatology of Aerodynamic Roughness Parameter and Energy Dissipation in the Planetary Boundary Layer of the Northern Hemisphere." In Annual Report 1963. Studies of the Effects of Variation in Boundary Conditions on the Atmospheric Boundary Layer. H. Lettau, (Ed.). University of Wisconsin, Madison.

Kung, E.C., R.A. Bryson, and D.H. Lenschow, 1964. "Study of a Continental Surface Albedo on the Basis of Flight Measurements and Structure of the Earth's Surface Ccver over North America," Monthly Weather Review, Vol. 92, pp. 543-564.

Laechelt, R.L. and B.M. Williams, 1976. "Value of Tree Shade to Homeowners," Alabama Forestry Commission, Montgomery, AL.

Landsberg, H.E. 1981. The Urban Climate, Academic Press, N.Y., 275 pp.

Lettau, H., 1969. "Note on Aerodynamic Roughness Parameter Estimation on the Basis of Roughness Element Description," Journal of Applied Meteorology, Vol. 3, pp. 828-832.

Maher, Y.; and Pielke, R.A. 1977. "A Numerical Study of the Air Flow over Irregular Terrain", Contr. Atmos. Phys., Vol. 50, pp. 98-113.

Mahrer, Y.; and Pielke, R.A. 1978. "A Test of an Upstream Splins: Interpolation Technique for the Advective Terms in a Numerical Mesoscale Model", Monthly Weather Review, Vol. 106, pp. 818-830.

Martien, P. H. Akbari, and A. Rosenfeld, 1989. "Light-Colored Surfaces to Reduce Suminertime Urban Temperatures: Btnefits, Costs, and Implementation issues," Presented at the 9th Miami International Congress On Energy and Environment, 11-13 December. 
McPherson, E.G., 1992. "Chicago's Evolving Urban Foresit an Initial Report to the Chicago Urban Forest Climate Project," Governnient Document No: A 13.42/2:C 43, $89 \mathrm{pp}$.

Monteith, J.L., 1975. Vegetation and the Atmosphere, Vol. 1, Academic Press, $278 \mathrm{pp}$.

Monteith, J.L., 1973. Principles of Environmental Physics, Edward Arnold, London, $241 \mathrm{pp}$.

Monteith, J.L., and G. Szeicz, 1961. "The Radiation Balance of Bare Soil and Vegetation," Quarterly Journal of the Royal Meteorological Society, Vol. 87, pp. 159-170.

Myrup, L.O., 1969. "A Numerical Model of the Urban Heat Island," Journal of Applied Meteorology, Vol. 8, pp. 908-918.

Oke, T.R., 1974. "Review of Urban Climatology, 1968-1973," W'MO Tech. Note No. 134, WMO No. 383, World Meteor. Organiz., Geneva.

Oke, T.R., 1979. "Review of Urban Climatology, 1973-1976," WMO Tech. Note No. 169, WMO No. 539, World Meteor. Organiz., Geneva.

Oke, T.R., 1987. Boundary Layer Climates, second edition, Methuen and Co. Ltd., London, $435 \mathrm{pp}$.

Outcalt, S.I., 1972a. "The Development and Application of a Simple Digita 1 Surface-Climate Simulator," Journal of Applied Meteorology, Vol. 11, pp. 629636.

Outcalt, S.I., 1972b. "A Reconnaissance Experiment in Mapping and Modeling the Effect of Land Use on Urban Thermal Regimes,"

Journal of Applied Meteorology, Vol. 11, pp. 1369-1373.

Parker, J.H., 1982. "Landscaping to Reduce the Energy used in cooling Buildings," Journal of Forestry, Vol. 81, No. 2, pp. 82-84.

Parker, J.H., 1989. "The Impact of Vegetation on Air Conditioning Consumption," in Proceedings of the workshop on saving energy and reducing atmospiteric pollution by controlling summer heat islands, Berkeley, California, pp. 45-51.

Pielke, R.A. 1984. Mesoscale meteorological modeling, Academic Press, Orlando, Florida, 612 pp. 
Reagan, J.A. and D.M. Acklam, 1979. "Solar Reflectivity of Common Building Materials and its Influence on the Roof Heat Gain of Typical Southwestern U.S.A. Residences," Energy and Buildings, pp. 237-238.

Renou, E., 1855. "Instructions M'et'eorologiques," Annuaire Soc. M'et 'eorol. de France, Vol. 3, Part 1, pp. 73-160.

Rosenfeld, A.H., H. Akbari, S. Bretz, D. Sailor, and H. Taha, 1993. "Mitigation of Urban Heat Islands: Materials, Utility Programs, Updates," Submitted to Journal of Energy Efficiency, 1993.

Ross, S.L. and T.R. Oke, 1988. "Tests of Three Urban Energy Balance Models," Boundary-Layer Meteor., Vol. 44, pp. 73-96.

Sailor, D.J., L. Rainer, and H. Akbari, 1992. "Measured Impact of Neighborhood Tree Cover on Microclimate," in Proceedings of the American Council for an Energy-Efficient Economy (ACEEE) 1992 Summer Study on Energy Efficiency in Buildings, Vol. 9, pp. 149-157.

Schmidt, W., 1927. "Die Verteilung der Minimumtemperaturen in der Frostnacht des 12.5. 1927 im Gemeindegebiet von Wien," Fortschr. Landwirtsch, Vol. 2 (H.21), pp. 681-686.

Schmidt, W., 1930. "Kleinklimatische Aufnahmen durch Temperaturfahrten," Meteorolog. Z., Vol. 47, pp. 92-106.

Schmugge, T., 1978. "Remote Sensing of Surface Soil Moisture," Journal of Applied Meteorology, Vol. 17, pp. 1549-1557.

Schultz, P; and Warner, T. 1982. "Characteristics of Summertime Circhlations and Pollutant Ventilation in the Los Angeles Basin", Journal of Applied Meteorology , Vol. 21, pp. 672-682.

Seaman, Nelson L., Francis L. Ludwig, Evelyn J. Donall, Thomas T. Warner, and Chandrakant M. Bhumralkar, 1989. "Numerical Studies of Urban Planetary Boundary-Layer Structure under Realistic Synoptic Conditions," Journal of Applied Meteorology, Vol. 28, pp. 760-781.

Sellers, W.D., 1965. Physical Climatology, University of Chicago Press, Chicago, $272 \mathrm{pp}$.

Sievers, U. and W.G. Zdunkowski, 1986. "A Microscale Urban Climate Model," Beitr. Phys. Atmosph., Vol. 59, pp. 13-40.

Stewart, J.B. 1971. "The Albedo of a Pine Forest," Quarterly Journal of the Royal Meteorological Society, Vol. 97, pp. 561-564. 
Taconet, O.R.. Bernard, and D. Vidal-Madjar, 1986. "Evapotranspiration over an Agricultural Regions Using a Surface Flux/Temperature Model Based on NOAA-AVHRR Data," Journal of Climate and Applied Meteorology, Vol. 25, No. 3, pp. 285-307.

Taha, H., H. Akbari, A. Rosenfeld, and Y.J. Huang, 1988. "Residential Cooling Loads and the Urban Heat Island: The Effects of Albedo," Building and Environment, Vol. 23, pp. 271-283.

Taha, H., H. Akbari, D. Sailor, 1992. "Causes and Effects of Heat Islands: The Sensitivity of Urban Microclimates to Surface Parameters and Anthropo. genic Heat", Lawrence Berkeley Laboratory report LBL-29864.

Taylor, P.A., 1987. "Comments and Further Analysis on Effective Roughness Lengths for use in Numerical Three-Dimensional Models," Boundary-Layer Meteorology, Vol. 39, pp. 403-418.

Terjung, W.H. and S.S-F. Louie, 1974. "A Climatic Model of Urban Energy Budgets," Geographical Analysis, Vol. 6, pp. 341-367.

Terjung, W.H. and P.A. O'Rourke, 1980. "Energy Exchanges in Urban Landscapes: Selected Climatic Models," Publications in Climatology, Vol. 33, No. 1.

Threlkeld, J.L., 1962. Thermal Environmental Engineering, Prentice-Hall International, London, $514 \mathrm{pp}$.

Ulrickson, Brian L., 1992. "Effects of Surface Property Variations on Simulated Daytime Airflow Over Coastal Southern California," Monthly Weather Review, Vol. 120, pp. 2264-2279.

U.S. Dept. of Commerce, 1980. DOE-2 Reference Manual, Parts 1 \& 2 (Version 2.1), Contract W-7405-ENG-8.

Vihma, T., and H. Savijarvi, 1991. "On the Effective Roughness Length for Heterogeneous Terrain," Quarterly Journal of the Royal Meteorological Society, Vol. 117, pp. 399-407.

Wechsler, A.E., and P.E. Glaser, 1966. "Surface Characteristics Effect on Thermal Regime: Phase I, Special report (88), U.S. Army CRREL, Hanover, New Hampshire.

Weiringa, J., 1986. "Roughness Dependent Geographical Interpolation of Surface Wind Speed Averages," Quarterly Journal of the Royal Meteorological Society, Vol. 112, pp. 867-889. 
Winer, A.M., J. Arey, S.M. Aschmann, R. Atkinson, W.D. Long, L.C. Morrison, and D.M. Olszyk, 1992. "Emission Rates of Organic Compounds from Agricultural and Natural Vegetation Found in California's Central Valley," Atmospheric Environment, in press.

Yoshikado, H. 1990. "Vertical Structure of the Sea Breeze Penetrating through a Large Urban Complex," Journal of Applied Meteorology, Vol. 29, pp. 878-891.

Yoshikado, H. 1992. "Numerical Study of the Daytime Urban Effect and Its Interaction with the Sea Breeze," Journal of Applied Meteorology, Vol. 31, pp. 1146-1164.

Yoshikado, H., and H. Kondo, 1989. "Inland Penetration of the Sea Breeze in the Suburban Area of Tokyo," Boundary-Layer Meteorology, Vol. 48, pp. 389-407. 

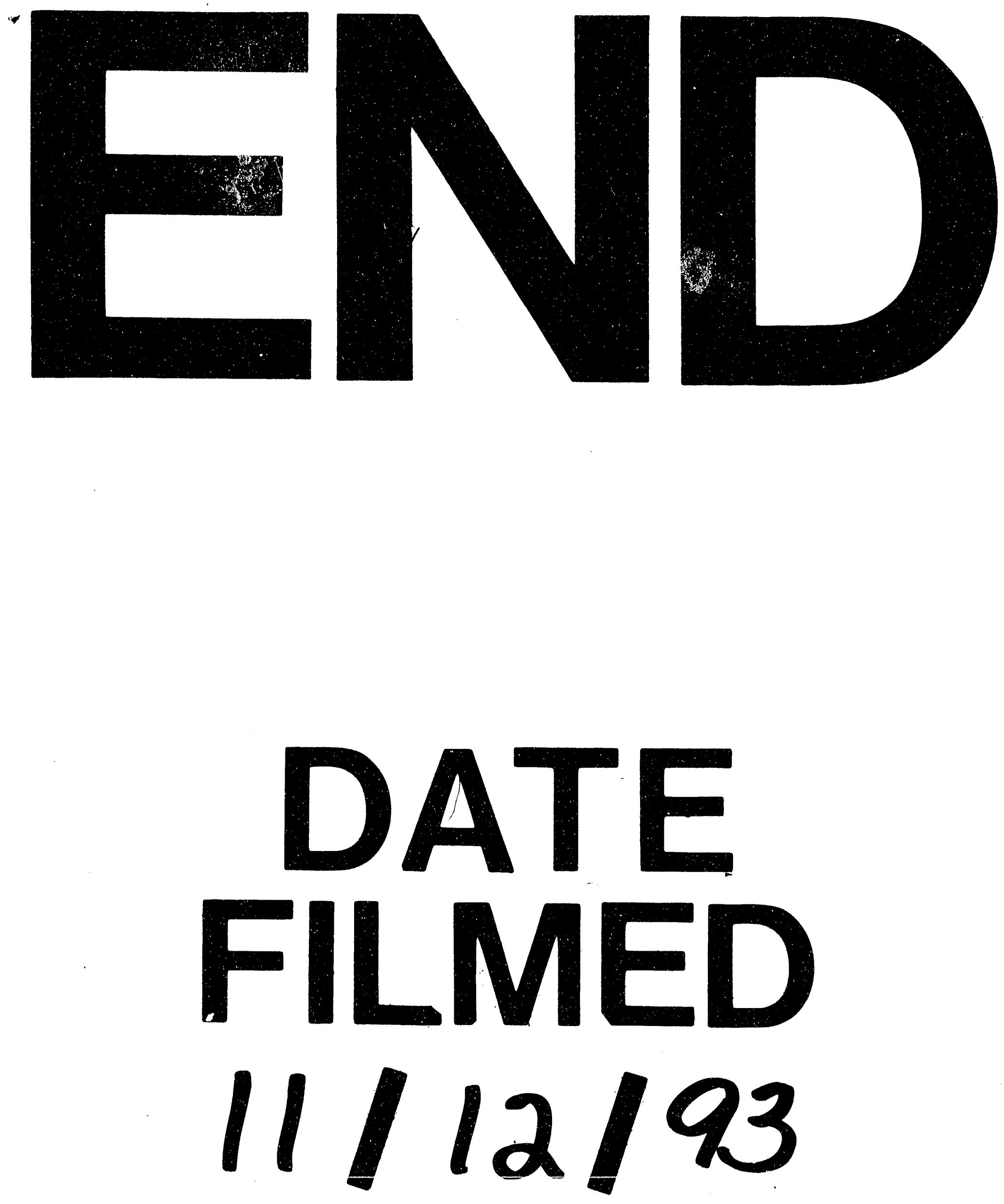
